TRANSACTIONS OF THE

AMERICAN MATHEMATICAL SOCIETY

Volume 358, Number 12, December 2006, Pages 5193-5256

S 0002-9947(06)04317-0

Article electronically published on July 21, 2006

\title{
BUMPY METRICS AND CLOSED PARAMETRIZED MINIMAL SURFACES IN RIEMANNIAN MANIFOLDS
}

\author{
JOHN DOUGLAS MOORE
}

\begin{abstract}
The purpose of this article is to study conformal harmonic maps $f: \Sigma \rightarrow M$, where $\Sigma$ is a closed Riemann surface and $M$ is a compact Riemannian manifold of dimension at least four. Such maps define parametrized minimal surfaces, possibly with branch points. We show that when the ambient manifold $M$ is given a generic metric, all prime closed parametrized minimal surfaces are free of branch points, and are as Morse nondegenerate as allowed by the group of automorphisms of $\Sigma$. They are Morse nondegenerate in the usual sense if $\Sigma$ has genus at least two, lie on two-dimensional nondegenerate critical submanifolds if $\Sigma$ has genus one, and on six-dimensional nondegenerate critical submanifolds if $\Sigma$ has genus zero.
\end{abstract}

\section{INTRODUCTION}

This article is devoted to providing part of the foundation needed for the development of a partial Morse theory for parametrized minimal surfaces in Riemannian manifolds, which should parallel the well-known Morse theory of smooth closed geodesics in a compact Riemannian manifold $M$. Recall that the theory of closed geodesics is concerned with the action integral

$$
J: L_{1}^{2}\left(S^{1}, M\right) \rightarrow \mathbb{R} \quad \text { defined by } \quad J(\gamma)=\frac{1}{2} \int_{S^{1}}\left\langle\gamma^{\prime}(t), \gamma^{\prime}(t)\right\rangle d t,
$$

where $\langle\cdot, \cdot\rangle$ denotes a Riemannian metric on $M$ and $t$ is the usual coordinate on $S^{1}$, which is regarded as the unit interval $[0,1]$ with endpoints identified. Moreover, $L_{1}^{2}\left(S^{1}, M\right)$ denotes the Hilbert manifold of $L_{1}^{2}$-maps of $S^{1}$ into $M$, maps which are $L^{2}$ and have $L^{2}$ first derivatives, as described for example in 20] or [19]. The function $J$ is invariant under a continuous right action of the circle group $S^{1}$ on $L_{1}^{2}\left(S^{1}, M\right)$,

$$
\phi: L_{1}^{2}\left(S^{1}, M\right) \times S^{1} \longrightarrow L_{1}^{2}\left(S^{1}, M\right), \quad \phi(\gamma, s)(t)=\gamma(t+s),
$$

and therefore whenever any point is critical for $J$, so is the entire $S^{1}$-orbit. Thus nonconstant critical points for $J$ are never Morse nondegenerate in the usual sense. However, the Bumpy Metric Theorem proven by Abraham [3] in 1970 states that for generic choice of Riemannian metric, all nonconstant smooth closed geodesics lie on nondegenerate critical submanifolds of dimension one, and have the property that their only Jacobi fields are those generated by the $S^{1}$-action.

The purpose of this article is to provide a similar bumpy metric theorem for the energy function on maps from compact Riemann surfaces without boundary into

Received by the editors February 18, 2004.

2000 Mathematics Subject Classification. Primary 53C40, 58E12; Secondary 58D15, 58E05.

(c)2006 American Mathematical Society Reverts to public domain 28 years from publication 
a compact manifold $M$. Here is the general setting. Suppose that $\Sigma$ is a compact Riemann surface without boundary of genus $g$, that $\operatorname{Map}(\Sigma, M)$ is an appropriate completion of the space of smooth maps from $\Sigma$ to $M$ with respect to a suitably strong Sobolev norm, and that $\mathcal{T}$ is the Teichmüller space of marked conformal structures on $\Sigma$. Then the energy $E: \operatorname{Map}(\Sigma, M) \times \mathcal{T} \rightarrow \mathbb{R}$ is defined by

$$
E(f, \omega)=\frac{1}{2} \int_{\Sigma}\left[\frac{\partial f}{\partial x} \cdot \frac{\partial f}{\partial x}+\frac{\partial f}{\partial y} \cdot \frac{\partial f}{\partial y}\right] d x d y=\frac{1}{2} \int_{\Sigma}|d f|^{2} d A,
$$

where $(x, y)$ are local conformal coordinates for the conformal structure $\omega \in \mathcal{T}$ (the integrand being independent of the choice) and $|d f|$ and $d A$ represent the norm of $d f$ and the area element with respect to any metric on $\Sigma$ within the conformal structure $\omega$. (We need to take the Sobolev norm on $\operatorname{Map}(\Sigma, M)$ strong enough so that the energy is defined, and we will in fact usually take this norm to be strong enough that $\operatorname{Map}(\Sigma, M)$ is a smooth Banach manifold.) For a fixed choice of $\omega \in \mathcal{T}$, we define

$$
E_{\omega}: \operatorname{Map}(\Sigma, M) \longrightarrow \mathbb{R} \quad \text { by } \quad E_{\omega}(f)=E(f, \omega) .
$$

Critical points for $E_{\omega}$ are called harmonic maps (or $\omega$-harmonic maps when we want to emphasize the specific conformal structure), while critical points for $E$ are (weakly) conformal harmonic maps, or equivalently minimal surfaces, possibly containing branch points. In Osserman's survey [29], in which the ambient manifold $M$ is Euclidean space, critical points of $E$ are called generalized minimal surfaces when branch points are allowed, but we will use the simpler term parametrized minimal surfaces for such objects.

In some cases one can prove existence of critical points for these functions by means of a perturbed energy introduced by Sacks and Uhlenbeck 31, 32. Thus for $\omega \in \mathcal{T}$ and $\alpha>1$, we define a function $E_{\alpha, \omega}: \operatorname{Map}(\Sigma, M) \rightarrow \mathbb{R}$ by

$$
E_{\alpha, \omega}(f)=\frac{1}{2} \iint_{\Sigma}\left(1+|d f|^{2}\right)^{\alpha} d A
$$

where the Riemannian metric on $\Sigma$ used in the integrand is chosen to be a metric within the conformal equivalence class defined by $\omega$. The ability to choose a metric on $\Sigma$ provides added flexibility to the method, but in this article, we will normalize the metric so that it is the unique constant curvature metric of total area one. We call $E_{\alpha, \omega}$ the $(\alpha, \omega)$-energy. We recall some important properties of the function $E_{\alpha, \omega}$ when $\operatorname{Map}(\Sigma, M)$ is taken to be the completion of the smooth maps from $\Sigma$ to $M$ with respect to the Sobolev $L_{1}^{p}$-norm, where $p=2 \alpha$ : The function $E_{\alpha, \omega}$ has continuous derivatives up to order two, it satisfies condition $\mathrm{C}$ of Palais and Smale and its critical points are smooth. For fixed choice of $\alpha>1$, one can use techniques of global analysis [37] to prove existence of critical points for $E_{\alpha, \omega}$ corresponding to minimax constraints. As $\alpha \rightarrow 1$, one can often show that a "minimax sequence" will approach a harmonic map in the limit, together with a finite number of "bubbles", which are harmonic maps of two-spheres.

There are several difficulties in constructing a Morse theory for the energy function $E$ via this approach. First, bubbling interferes with the most straightforward version of the Morse inequalities (but also suggests a procedure for analyzing to what extent the Morse inequalities fail). Second, minimax sequences for various constraints might tend to the boundary of Teichmüller space, a phenomenon already observed in the Plateau problem for mappings of Riemann surfaces with 
boundary into Euclidean space $\mathbb{R}^{3}$ — thus a minimizing sequence might have a handle pinch off in the limit, for example. Third, a given minimal surface has infinitely many different markings, giving rise to an orbit of critical points in $\operatorname{Map}(\Sigma, M) \times \mathcal{T}$ under an action of the mapping class group $\Gamma$. (We describe this action at the beginning of 95 ) This action leaves the energy invariant, so the energy actually descends to a function on the quotient

$$
E:(\operatorname{Map}(\Sigma, M) \times \mathcal{T}) / \Gamma \rightarrow \mathbb{R}
$$

where the domain projects to the moduli space $\mathcal{R}=\mathcal{T} / \Gamma$ with typical fiber $\operatorname{Map}(\Sigma, M)$. One really wants Morse theory to estimate the number of critical points of given index in the quotient, and although the base space $\mathcal{R}$ is diffeomorphic to a ball when $g=1$, it has a much-studied but complicated topology when $g>1$.

Finally, just as in the theory of smooth closed geodesics, multiple covers of a given minimal surface should not count as geometrically distinct, even though they will appear as distinct critical points for $E$. Indeed, this difficulty becomes more pronounced when one passes from maps from curves to maps from surfaces because the covers might be branched: Suppose, for example, that $h: \Sigma_{2} \rightarrow M$ is a smooth harmonic map and that $g: \Sigma_{1} \rightarrow \Sigma_{2}$ is a nontrivial conformal branched cover. (If $\Sigma_{2}=S^{2}$, the Riemann sphere, we can regard $g$ as a meromorphic function on $\Sigma_{1}$.) Then the composition $f=h \circ g: \Sigma_{1} \rightarrow M$ is called a branched cover of the harmonic map $h: \Sigma_{2} \rightarrow M$. Any compact simply connected Riemannian manifold must contain minimal two-spheres [31, so branched covers are present in many of the most interesting cases.

In spite of these difficulties, it is sometimes possible to control bubbling and use critical point theory for the $\alpha$-energy to prove existence of harmonic maps or minimal surfaces. One application is the theorem of Sacks and Uhlenbeck 31. that any compact simply connected Riemannian manifold contains at least one minimal two-sphere. Another application involving equivariant Morse theory for minimal two-spheres is presented in [28, in which we use bounds on the energy to prevent bubbling, and thereby prove existence of many minimal two-spheres of low energy in suitably pinched $n$-spheres, which satisfy appropriate hypotheses. We hope to present further applications in subsequent articles, but need a bumpy metric theorem for parametrized minimal surfaces as a foundation.

In view of transversality techniques of Smale [35] and Uhlenbeck [36], it is not surprising that such a bumpy metric theorem should hold when properly formulated. Indeed, White proves a bumpy metric theorem for imbedded minimal submanifolds of arbitrary dimension in a Riemannian ambient manifold 38. However, for the theory of parametrized minimal surfaces, it is crucial that we allow for the possibility of branch points. Branch points always occur on the nontrivial branched covers of parametrized surfaces of lower genus mentioned before; such branched covers can have arbitrarily large spaces of Jacobi fields that cannot be removed by generic perturbations of the metric on $M$. Removing branch points by perturbation on the remaining surfaces is the key issue which needs to be resolved for establishing a bumpy metric theorem in the parametrized context which is strong enough for our projected applications. (We should point out that there is a related transversality theorem for Gromov's theory of pseudoholomorphic curves which is presented in the book of McDuff and Salamon [23].) 
Another complication to the parametrized theory is that when the genus of $\Sigma$ is small, the energy function $E$ is invariant under a nontrivial connected Lie group $G$ of symmetries. Thus in the case where $\Sigma$ is the torus, the two-dimensional Lie group $G=S^{1} \times S^{1}$ acts on $\operatorname{Map}(\Sigma, M)$ preserving the energy, which implies that nonconstant minimal tori lie on orbits of critical points of dimension two. In the case where $\Sigma$ is the Riemann sphere $S^{2}$, the energy is invariant under the action of the six-dimensional Lie group $G=P S L(2, \mathbb{C})$ via linear fractional transformations on the domain. This action preserves the energy and hence nonconstant minimal two-spheres lie on orbits of critical points having dimension six. Since $\operatorname{PSL}(2, \mathbb{C})$ is noncompact, it is often convenient (in accordance with a procedure adopted in [28] to replace $\operatorname{Map}\left(S^{2}, M\right)$ by the subspace of maps with "center of mass zero",

$$
\operatorname{Map}_{0}\left(S^{2}, M\right)=\left\{f \in \operatorname{Map}\left(S^{2}, M\right): \int_{S^{2}} X|d f|^{2} d A=0\right\},
$$

where $X: S^{2} \rightarrow \mathbb{R}^{3}$ is the standard inclusion onto the unit sphere in $\mathbb{R}^{3}$. This allows us to reduce the symmetry group of the energy function from $\operatorname{PSL}(2, \mathbb{C})$ to its maximal compact subgroup $S O(3)$. (This has many technical advantages and is useful for the approach to existence via $\alpha$-energy, because the $\alpha$-energy is only invariant under the smaller group $S O(3)$.) Because of the group actions, the most one could hope for in the cases of minimal spheres and tori (as well as nonorientable projective planes and Klein bottles) is that in analogy with the closed geodesic problem, the critical points for energy lie on nondegenerate critical submanifolds for generic choice of metric on the ambient manifold. Let us recall the definition (due to Bott):

Definition. Let $F: \mathcal{M} \rightarrow \mathbb{R}$ be a $C^{2}$ function on a smooth manifold $\mathcal{M}$. A nondegenerate critical submanifold of $\mathcal{M}$ is a finite-dimensional submanifold $N \subset$ $\mathcal{M}$ such that every $f \in N$ is a critical point for $F$ and

$$
f \in N \quad \Rightarrow \quad T_{f} N=\left\{X \in T_{f} \mathcal{M}: d^{2} F(f)(X, Y)=0 \text { for all } Y \in T_{f} \mathcal{M}\right\} .
$$

Here $d^{2} F$ is the Hessian of $F$ at the critical point, and elements $X \in T_{f} \mathcal{M}$ which satisfy the condition on the right-hand side of (3) are called Jacobi fields.

Definition. We say that a parametrized minimal surface $f: \Sigma \rightarrow M$ is prime if it is nonconstant and is not a nontrivial cover (possibly branched) of another parametrized minimal surface $f_{0}: \Sigma_{0} \rightarrow M$ of lower energy, where we explicitly allow the surface $\Sigma_{0}$ to be nonorientable.

Definition. By a generic choice of Riemannian metric, we mean a metric belonging to a countable intersection of open dense subsets of the spaces of $L_{k}^{2}$ Riemannian metrics on $M$, where $k$ ranges over the positive integers.

With these definitions in place, we can now state our main theorem for critical points of the energy function $E$ :

Main Theorem. Suppose that $M$ is a compact connected smooth manifold of dimension at least four. Then

(1) For a generic choice of Riemannian metric on $M$, every prime minimal two-sphere $f: S^{2} \rightarrow M$ is free of branch points and lies on a nondegenerate critical submanifold of dimension six which is an orbit for the G-action, where $G=P S L(2, \mathbb{C})$. 
(2) For a generic choice of Riemannian metric on $M$, every prime minimal two-torus $f: T^{2} \rightarrow M$ is free of branch points and lies on a nondegenerate critical submanifold of dimension two which is an orbit for the G-action, where $G=S^{1} \times S^{1}$.

(3) For a generic choice of Riemannian metric on $M$, every prime oriented minimal surface $f: \Sigma \rightarrow M$ of genus at least two is free of branch points and is Morse nondegenerate in the usual sense.

In particular, if $M$ has a generic metric, prime oriented closed parametrized minimal surfaces within $M$ are immersions. For simplicity, we will focus on oriented minimal surfaces throughout most of the article, although the argument can be modified to give a bumpy metric theorem for nonorientable minimal surfaces by arguments we sketch in \$11.

The freedom from branch points asserted in the Main Theorem provides an ana$\log$ of a well-known theorem of Böhme and Tromba ( 8 and 9$]$ ) that parametrized minimal surfaces bounded by a generic smooth closed Jordan curve in $\mathbb{R}^{n}$ have no branch points when $n \geq 4$. Note, moreover, that the first case of the Main Theorem shows that the nondegeneracy hypothesis of Theorems 1 and 2 of 28 hold for generic metrics.

Here is a brief outline of the remainder of the article. We first review the parametric theory of closed harmonic and minimal surfaces in Riemannian manifolds in \$2 and explain the relationship with the theory of Riemann surfaces. We next describe how the Sard-Smale Theorem yields a bumpy metric theorem for $\omega$-harmonic surfaces which satisfy additional hypotheses in $\$ 3$, we use the argument as a model for the proof of the Main Theorem. In $\$ 4$, we describe the possible self-intersections of prime parametrized minimal surfaces in a compact Riemannian manifold of dimension at least three; the full force of this description will not be needed until \$7. To treat minimal surfaces of arbitrary genus, we need a second variation formula for the two-variable energy in which the conformal structure is free to vary. This second variation formula is presented in \$5 and then applied to yield a bumpy metric theorem for minimal surfaces without branch points in 86 .

We present the key lemma in $\$ 7$ This lemma is used to perturb away some of the extra Jacobi fields contributed by branch points, and we show how it can be applied to eliminate simple branch points via a suitable perturbation of the metric in 88 . To treat branch points which are not simple, we next describe a branch point stratification in 99. We then finish the proof of the Main Theorem in 10 . In $\$ 11$ we describe how the argument for the Main Theorem can be modified to treat prime nonorientable minimal surfaces, while a brief final section (\$12) describes how the Main Theorem can be extended to generic one-parameter families of metrics on $M$.

Throughout this article, we will assume some familiarity with the techniques of global analysis on infinite-dimensional manifolds, as presented especially in [2, but with a far more detailed treatment in [30. More leisurely introductions are found in 21] and [4], for example. One of the core theorems to keep in mind is nicely stated in a survey article by Eells [13. (see page 780): If $S$ and $M$ are finite-dimensional smooth manifolds and $C^{k}(S, M)$ denotes the completion of the space of smooth maps from $S$ to $M$ with the $C^{k}$ norm, a smooth infinite-dimensional manifold, then

$$
\Phi: C^{k+s}(M, N) \times C^{k}(S, M) \rightarrow C^{k}(S, N), \quad \Phi(g, f)=g \circ f
$$


is a $C^{s}$ map. This specializes to yield versions of the so-called $\alpha$ - and $\omega$-Lemmas. One version of the $\omega$-Lemma for $L_{k}^{p}$ spaces states that if $\Sigma$ is a smooth surface and $M_{1}$ and $M_{2}$ are smooth manifolds of arbitrary dimension, then a $C^{\infty}$ map $g: M_{1} \rightarrow M_{2}$ induces a $C^{\infty}$ map

$$
\omega_{g}: L_{k}^{p}\left(\Sigma, M_{1}\right) \longrightarrow L_{k}^{p}\left(\Sigma, M_{2}\right), \quad \omega_{g}(f)=g \circ f
$$

where $L_{k}^{p}(\Sigma, M)$ denotes the completion of $C^{\infty}$ maps with respect to the $L_{k}^{p}$ norm, for any $p$ and $k$ satisfying $k-2 / p>0$. On the other hand, the $\alpha$-Lemma presented in $\S 11$ of [2] states that a $C^{\infty}$ map $h: \Sigma_{1} \rightarrow \Sigma_{2}$ induces a $C^{\infty}$ map

$$
\alpha_{h}: C^{k}\left(\Sigma_{2}, M\right) \longrightarrow C^{k}\left(\Sigma_{1}, M\right), \quad \alpha_{h}(f)=f \circ h .
$$

The loss of derivatives implicit in (4) and appearing in related theorems often forces us to work in a Sobolev completion $L_{k}^{2}(\Sigma, M)$ in which the number $k$ of generalized derivatives is high. For example, in arguments involving branch points the value of $k$ we need is sometimes determined by the largest possible branching order, which in turn depends on an upper bound $E_{0}$ for the energy, as we will see in the next section.

The author takes pleasure in thanking Vincent Borrelli of the Université Claude Bernard of Lyon, France, for helpful discussions on the theory presented here during a visit to Lyon in December of 2004. He also thanks the referee for numerous questions and suggestions that helped him make many major improvements to the article.

\section{Preliminaries}

We need to recall a few basic concepts from the theory of harmonic and minimal surfaces in Riemannian manifolds (some of which are presented in more detail in [26]). We focus first on the $\omega$-energy $E_{\omega}$ for a fixed choice of Riemannian metric $g=\langle\cdot, \cdot\rangle$ on $M$. The first derivative of $E_{\omega}$ is given by the formula

$$
d E_{\omega}(f)(X)=\int_{\Sigma}\left\langle F_{\omega}(f, g), X\right\rangle d A,
$$

where $F_{\omega}(\cdot, g)=0$ is the Euler-Lagrange equation for the variational problem. If $f$ is a critical point for $E_{\omega}$, we can differentiate once again to obtain the Hessian,

$$
d^{2} E_{\omega}(f)(X, Y)=\int_{\Sigma}\left\langle D_{1} F_{\omega}(f, g)(X), Y\right\rangle d A=\int_{\Sigma}\left\langle L_{(f, g)}(X), Y\right\rangle d A,
$$

where $D_{1} F_{\omega}$ denotes the derivative with respect to the variable $f \in \operatorname{Map}(\Sigma, M)$ and $L_{(f, g)}$ is the Jacobi operator, a formally self-adjoint second-order elliptic differential operator which acts on sections of the pullback $f^{*} T M$ of the tangent bundle to $M$. Such sections are the elements of the tangent space to $\operatorname{Map}(\Sigma, M)$ at $f$. Elements $X$ of this tangent space $T_{f} \operatorname{Map}(\Sigma, M)$ which satisfy the Jacobi equation $L_{(f, g)}(X)=0$ are called Jacobi fields at $f$. Note that since $L_{(f, g)}=D_{1} F_{\omega}(f, g)$, the Jacobi equation is just the linearization of the Euler-Lagrange equation.

In terms of a local complex coordinate $z=x+i y$ on $\Sigma$, with $\lambda^{2}$ denoting the conformal factor (so that the area element is given by $d A=\lambda^{2} d x d y$ ), the EulerLagrange equation for an $\omega$-harmonic map $f: \Sigma \rightarrow M$ is simply

$$
-\frac{1}{4 \lambda^{2}} \frac{D}{\partial \bar{z}}\left(\frac{\partial f}{\partial z}\right)=0, \quad \text { where } \quad \frac{\partial f}{\partial z}=f_{*}\left(\frac{\partial}{\partial z}\right)
$$


is regarded as a section of the complex bundle $\mathbf{E}=f^{*} T M \otimes \mathbb{C}$ over $\Sigma$, and $D$ denotes the pullback of the Levi-Civita connection of $M$ to E. Thus the Euler-Lagrange equation just says that the section $\partial f / \partial z$ is holomorphic, when $\mathbf{E}$ is given the holomorphic structure (guaranteed to exist by a theorem of Koszul and Malgrange) such that if $V$ is an element of $\Gamma(\mathbf{E})$, the space of smooth sections of $\mathbf{E}$, then

$$
V \text { is holomorphic } \quad \Leftrightarrow \quad \frac{D V}{\partial \bar{z}}=0 .
$$

Although the locally defined holomorphic section

$$
\frac{\partial f}{\partial z} \text { may have isolated zeros, the quotient } \quad\left[\frac{\partial f}{\partial z}\right]: \Sigma \rightarrow \mathbb{P}(\mathbf{E})
$$

is independent of choice of local coordinate $z$ and is a globally defined section on $\Sigma$, where $\mathbb{P}(\mathbf{E})$ denotes the bundle of complex projective lines in fibers of $\mathbf{E}$. This globally defined family of lines defines a holomorphic line bundle $\mathbf{L}$ within $\mathbf{E}$. A point $p \in \Sigma$ is said to be a branch point if $(\partial f / \partial z)(p)=0$. In this case, if $z$ is a holomorphic coordinate centered at $p$, we can write

$$
\frac{\partial f}{\partial z}=z^{\nu} g(z)
$$

where $g$ is a holomorphic section of $\mathbf{E}$ such that $g(p) \neq 0$. The integer $\nu$ is called the branching order of $f$ at $p$.

Observe that the vector-valued differential

$$
\partial f=\frac{\partial f}{\partial z} d z \quad \text { is a globally defined holomorphic section of } \mathbf{L} \otimes \mathbf{K},
$$

where $\mathbf{K}=T_{h}^{*} \Sigma$ is the holomorphic cotangent bundle of $\Sigma$, also known as the canonical bundle, and this section has zeros precisely at the branch points of $f$. In particular, if $f$ has no branch points, this section trivializes $\mathbf{L} \otimes \mathbf{K}$, and we conclude that $\mathbf{L}$ must be isomorphic to the holomorphic line bundle $\mathbf{K}^{*}$ dual to $\mathbf{K}$, that is, to the holomorphic tangent bundle to $\Sigma$. In the general case, we let $\nu_{p}(f)$ denote the branching order of the harmonic map $f$ at $p$ and call the finite sum

$$
D(f)=\sum\left\{\nu_{p}(f) p: \nu_{p}(f)>0\right\}
$$

the divisor of the harmonic map $f$. By standard arguments in Riemann surface theory (as presented in [18, $\S 7$ ), we can determine the isomorphism class of the holomorphic bundle $\mathbf{L}$, the result being that

$$
\mathbf{L} \otimes \mathbf{K} \cong \zeta_{p_{1}}^{\nu_{p_{1}}} \otimes \cdots \otimes \zeta_{p_{n}}^{\nu_{p_{n}}}, \quad \text { and hence } \quad \mathbf{L} \cong \mathbf{K}^{*} \otimes \zeta_{p_{1}}^{\nu_{p_{1}}} \otimes \cdots \otimes \zeta_{p_{n}}^{\nu_{p_{n}}},
$$

where $p_{1}, \ldots, p_{n}$ are the branch points of $f$ and $\zeta_{p}$ is the holomorphic point bundle at $p$. The Kronecker product of the first Chern class of $\mathbf{L}$ and the fundamental class of $\Sigma$ is given by the formula

$$
\left\langle c_{1}(\mathbf{L}),[\Sigma]\right\rangle=\left\langle c_{1}\left(\mathbf{K}^{*}\right),[\Sigma]\right\rangle+\sum \nu_{p}=(2-2 g)+\sum \nu_{p},
$$

where $\sum \nu_{p}$ is the total branching order of $f$.

In the case of the sphere $S^{2}=\mathbb{C} \cup\{\infty\}$ we can take the standard complex coordinate $z$ on $\mathbb{C}$ and check that

$$
\frac{\partial f}{\partial z} \text { extends to a holomorphic vector field which vanishes at } \infty \text {. }
$$


Thus the holomorphic function

$$
\left\langle\frac{\partial f}{\partial z}, \frac{\partial f}{\partial z}\right\rangle \text { is globally defined and vanishes at } \infty,
$$

and must therefore be identically zero. By dividing into real and imaginary parts, we conclude that

$$
\left\langle\frac{\partial f}{\partial x}, \frac{\partial f}{\partial x}\right\rangle=\left\langle\frac{\partial f}{\partial y}, \frac{\partial f}{\partial y}\right\rangle, \quad\left\langle\frac{\partial f}{\partial x}, \frac{\partial f}{\partial y}\right\rangle=0,
$$

which is just the condition that the harmonic two-sphere $f$ be conformal. (Some authors would say weakly conformal when $f$ has branch points.)

In the case of the torus, the coordinate vector field

$$
\frac{\partial f}{\partial z} \quad \text { is globally defined and } \quad\left\langle\frac{\partial f}{\partial z}, \frac{\partial f}{\partial z}\right\rangle
$$

is a globally defined holomorphic function, which must therefore be constant by the maximum modulus principle. The constant is zero precisely when $f$ is conformal. Since the constant is nonzero for a nonconformal harmonic torus, such a torus cannot have branch points.

To deal with Riemann surfaces of genus $g \geq 2$ we utilize the Hopf differential

$$
\Omega_{f}=\langle\partial f, \partial f\rangle=\left\langle\frac{\partial f}{\partial z}, \frac{\partial f}{\partial z}\right\rangle d z^{2},
$$

a quadratic differential which is holomorphic when $f$ is $\omega$-harmonic and vanishes when $f$ is also conformal. If $\Sigma$ has genus $g \geq 2$, all nonzero holomorphic quadratic differentials on $\Sigma$ must have the same number of zeros counting multiplicity, namely

$$
\left\langle c_{1}\left(\mathbf{K}^{2}\right),[\Sigma]\right\rangle=\left\langle c_{1}\left(T_{h}^{*} \Sigma \otimes T_{h}^{*} \Sigma\right),[\Sigma]\right\rangle=4 g-4 .
$$

On the other hand, if a nonconformal $\omega$-harmonic map $f: \Sigma \rightarrow M$ has a branch point of branching order $k$ at $p$, then the Hopf differential $\Omega_{f}$ must have a zero of order at least $2 k$ at $p$, and hence

$$
\nu(f)=(\text { total branching order of } f) \leq \frac{1}{2}\left(\text { number of zeroes of } \Omega_{f}\right)=2 g-2 .
$$

Thus a nonconformal harmonic surface of genus $g \geq 2$ cannot have more than $2 g-2$ branch points, counting multiplicity. Moreover,

$$
\left\langle c_{1}(\mathbf{L}),[\Sigma]\right\rangle=2-2 g+\nu(f) \leq 0,
$$

so $\mathbf{L}$ has no holomorphic sections, unless it is the trivial line bundle and $\nu(f)=$ $2 g-2$. The case where $\mathbf{L}$ is the trivial bundle can occur if $f=h \circ g$, where $g: \Sigma \rightarrow T^{2}$ is a branched cover and $h: T^{2} \rightarrow M$ is a nonconformal harmonic torus.

Returning to the case of general genus, we note that if $f$ is not only harmonic but also conformal, the rank of $d f_{p}$ can never be one, and $f$ is an immersion except at branch points. This contrasts with the fact that nonconformal harmonic maps can have points at which $d f_{p}$ has rank one; this happens for example when $f$ is a torus parametrization of a geodesic. If $f$ is an immersion except for branch points, the real and imaginary parts of sections of the line bundle $\mathbf{L}$ generate a two-dimensional subbundle $\left(f^{*} T M\right)^{\top}$ of the pullback tangent bundle $f^{*} T M$. This subbundle has an induced metric and orientation which determine an almost complex structure

$$
J: \Gamma\left(\left(f^{*} T M\right)^{\top}\right) \rightarrow \Gamma\left(\left(f^{*} T M\right)^{\top}\right),
$$


where $\Gamma\left(\left(f^{*} T M\right)^{\top}\right)$ denotes the space of smooth sections of $\left(f^{*} T M\right)^{\top}$, such that

$$
\mathbf{L} \cong\left\{v \in\left(f^{*} T M\right)^{\top} \otimes \mathbb{C}: J(v)=i v\right\}, \quad \mathbf{L}^{*} \cong\left\{v \in\left(f^{*} T M\right)^{\top} \otimes \mathbb{C}: J(v)=-i v\right\} .
$$

More explicitly, the map $\tau:\left(f^{*} T M\right)^{\top} \rightarrow \mathbf{L}$ defined by

$$
\tau\left(M \frac{\partial f}{\partial x}+N \frac{\partial f}{\partial y}\right)=2(M+i N) \frac{\partial f}{\partial z}
$$

is a complex linear isomorphism, the inverse of which is just the projection of a section of $\mathbf{L}$ to its real part. We will use this isomorphism repeatedly in the sequel.

The bundle $\left(f^{*} T M\right)^{\top}$ has an orthogonal complement $\left(f^{*} T M\right)^{\perp}$ and we can define the second fundamental form

$$
A(f): \Gamma\left(f^{*} T M\right)^{\top} \times \Gamma\left(f^{*} T M\right)^{\top} \rightarrow \Gamma\left(f^{*} T M\right)^{\perp} \quad \text { by } \quad A(f)(X, Y)=\left(D_{X} Y\right)^{\perp},
$$

with $D$ denoting once again the Levi-Civita connection. It follows immediately from the equation for harmonic maps (7) that

$$
A(f)\left(\frac{\partial f}{\partial x}, \frac{\partial f}{\partial x}\right)+A(f)\left(\frac{\partial f}{\partial y}, \frac{\partial f}{\partial y}\right)=0 .
$$

If we normalize the Riemannian metric on the compact manifold $M$ so that the sectional curvatures of $M$ are $\leq 1$, the Gauss equations together with (10) imply that the curvature $K$ of $\left(f^{*} T M\right)^{\top}$ is also $\leq 1$, and hence that

$$
\left\langle c_{1}(L),[\Sigma]\right\rangle=\frac{1}{2 \pi} \int_{\Sigma} K d A \leq \frac{1}{2 \pi} \int_{\Sigma} d A=\frac{\text { Area of } f}{2 \pi} \leq \frac{\text { Energy of } f}{2 \pi} .
$$

It follows from (11) that minimal spheres of sufficiently small energy cannot have branch points, and the total branching order of a parametrized minimal surface of genus $g$ grows at most linearly with the energy. Thus given a bound $E_{0}$ on the energy, we can bound the total branching order $\nu(f)$.

Let $\operatorname{Met}(M)$ denote the space of Riemannian metrics on $M$, completed with respect to a suitable Sobolev norm. Given an element $\omega$ of the Teichmüller space $\mathcal{T}$ for the closed oriented surface $\Sigma$ of genus $g$, we let

$$
\mathcal{S}_{\omega}=\left\{(f, g) \in \operatorname{Map}(\Sigma, M) \times \operatorname{Met}(M): d f_{p}\right. \text { is injective }
$$

$$
\text { for some } p \in \Sigma \text { and } f \text { is } \omega \text {-harmonic for the metric } g \text { \}. }
$$

We also let

$$
\mathcal{S}=\{(f, \omega, g) \in \operatorname{Map}(\Sigma, M) \times \mathcal{T} \times \operatorname{Met}(M):
$$

$f$ is both weakly conformal and $\omega$-harmonic for the metric $g$.

In analogy with the presentation in Böhme and Tromba [8, 9], we can construct stratifications of $\mathcal{S}_{\omega}$ and $\mathcal{S}$, the strata being

$$
\begin{gathered}
\mathcal{S}_{\omega}^{\nu}=\left\{(f, g) \in \mathcal{S}_{\omega}: f \text { has total branching order } \nu\right\}, \\
\mathcal{S}^{\nu}=\{(f, \omega, g) \in \mathcal{S}: f \text { has total branching order } \nu\},
\end{gathered}
$$

where, of course, $\nu$ is a nonnegative integer.

Holomorphic sections of the line bundle $\mathbf{L}$ can be identified with meromorphic sections $X$ of the holomorphic tangent bundle $T_{h} \Sigma$ which have the property that

$$
(X)=(\text { divisor of } X) \geq-\sum \nu_{p} p=-(\text { divisor of } f) \text {. }
$$


The space $\mathcal{O}(\mathbf{L})$ of such sections is easily determined when $\Sigma$ has genus either zero or one. If $\Sigma$ has genus zero, it follows from the Riemann-Roch theorem that

$$
(f, g) \in \mathcal{S}_{\omega}^{\nu} \quad \text { or } \quad(f, \omega, g) \in \mathcal{S}^{\nu} \quad \Rightarrow \quad \operatorname{dim}_{\mathbb{C}} \mathcal{O}(\mathbf{L})=\nu+3,
$$

there being only one conformal structure $\omega$ in this case. If $\Sigma$ has genus one, the lowest degree stratum $\mathcal{S}^{0}$ only occurs when $\mathbf{L}$ is trivial and $\operatorname{dim}_{\mathbb{C}} \mathcal{O}(\mathbf{L})=1$, as forced by the action of $S^{1} \times S^{1}$. Moreover, if $\nu \geq 1$, it follows from the Riemann-Roch theorem that

$$
(f, g) \in \mathcal{S}_{\omega}^{\nu} \quad \text { or } \quad(f, \omega, g) \in \mathcal{S}^{\nu} \quad \Rightarrow \quad \operatorname{dim}_{\mathbb{C}} \mathcal{O}(\mathbf{L})=\nu .
$$

The Jacobi operator, defined in terms of the second variation by (6), plays a central role in this article; we review the complex version of this operator as presented in [26]. It follows from the usual second variation formula for harmonic maps that the Jacobi operator has the explicit expression

$$
L_{(f, g)}(\cdot)=-\frac{1}{\lambda^{2}}\left[\frac{D}{\partial x} \circ \frac{D}{\partial x}+\frac{D}{\partial y} \circ \frac{D}{\partial y}+R\left(\cdot, \frac{\partial f}{\partial x}\right) \frac{\partial f}{\partial x}+R\left(\cdot, \frac{\partial f}{\partial y}\right) \frac{\partial f}{\partial y}\right],
$$

where $R$ is the Riemann-Christoffel curvature tensor of $M$. Straightforward calculations show that on the one hand,

$$
\begin{aligned}
4 \frac{D}{\partial z} \circ \frac{D}{\partial \bar{z}}=\frac{D}{\partial x} \circ \frac{D}{\partial x}+\frac{D}{\partial y} \circ \frac{D}{\partial y} & +\sqrt{-1}\left(\frac{D}{\partial x} \circ \frac{D}{\partial y}-\frac{D}{\partial y} \circ \frac{D}{\partial x}\right) \\
& =\frac{D}{\partial x} \circ \frac{D}{\partial x}+\frac{D}{\partial y} \circ \frac{D}{\partial y}+\sqrt{-1} R\left(\frac{\partial f}{\partial x}, \frac{\partial f}{\partial y}\right),
\end{aligned}
$$

while on the other hand, if $R$ is extended to be complex linear,

$$
\begin{aligned}
4 R\left(\cdot, \frac{\partial f}{\partial z}\right) \frac{\partial f}{\partial \bar{z}}= & R\left(\cdot, \frac{\partial f}{\partial x}\right) \frac{\partial f}{\partial x}+R\left(\cdot, \frac{\partial f}{\partial y}\right) \frac{\partial f}{\partial y} \\
+ & \sqrt{-1}\left[R\left(\cdot, \frac{\partial f}{\partial x}\right) \frac{\partial f}{\partial y}-R\left(\cdot, \frac{\partial f}{\partial y}\right) \frac{\partial f}{\partial x}\right] \\
& =R\left(\cdot, \frac{\partial f}{\partial x}\right) \frac{\partial f}{\partial x}+R\left(\cdot, \frac{\partial f}{\partial y}\right) \frac{\partial f}{\partial y}-\sqrt{-1} R\left(\frac{\partial f}{\partial x}, \frac{\partial f}{\partial y}\right),
\end{aligned}
$$

with the last step following from the Bianchi symmetry. We conclude that the Jacobi equation, or the linearization of the Euler-Lagrange equation (7) at an $\omega$ harmonic map $f$, is

$$
L_{(f, g)}(Z)=0, \quad \text { where } \quad L_{(f, g)}=-\frac{4}{\lambda^{2}}\left[\frac{D}{\partial z} \circ \frac{D}{\partial \bar{z}}+R\left(\cdot, \frac{\partial f}{\partial z}\right) \frac{\partial f}{\partial \bar{z}}\right] .
$$

Integration by parts shows that the Hessian of the $\omega$-energy at an $\omega$-harmonic map $f$ extends to the symmetric complex bilinear form

$$
d^{2} E_{\omega}(f): \Gamma(\mathbf{E}) \times \Gamma(\mathbf{E}) \longrightarrow \mathbb{C}, \quad \text { where } \quad \mathbf{E}=f^{*} T M \otimes \mathbb{C},
$$

which satisfies the formula

$$
\begin{aligned}
d^{2} E_{\omega}(f)(Z, \bar{Z})=4 \int_{\Sigma} & {\left[\left|\frac{D Z}{\partial \bar{z}}\right|^{2}-\left\langle R\left(Z, \frac{\partial f}{\partial z}\right) \frac{\partial f}{\partial \bar{z}}, \bar{Z}\right\rangle\right] d x d y } \\
& =4 \int_{\Sigma}\left[\left|\frac{D Z}{\partial \bar{z}}\right|^{2}-\left\langle\mathcal{R}\left(Z \wedge \frac{\partial f}{\partial z}\right), \bar{Z} \wedge \frac{\partial f}{\partial \bar{z}}\right\rangle\right] d x d y
\end{aligned}
$$


with $\bar{Z}$ denoting the complex conjugate of $Z$. Here $\langle\cdot, \cdot\rangle$ denotes the complex bilinear extension of the Riemannian metric on $M$ and $\mathcal{R}: \Lambda^{2} \mathbf{E} \rightarrow \Lambda^{2} \mathbf{E}$ is the complex linear extension of the curvature operator.

It follows directly from these formulae that a holomorphic section of $\mathbf{L}$ is automatically a Jacobi field (since the curvature term vanishes for sections of $\mathbf{L}$ ). It is easily checked that a section $X$ of $\left(f^{*} T M\right)^{\top}$ is a Jacobi field if and only if the corresponding section $\tau(X)$ of $\mathbf{L}$ is also a Jacobi field. Thus for an $\omega$-harmonic map $f$, the real dimension of the space of Jacobi fields which are sections of $\left(f^{*} T M\right)^{\top}$ is twice the complex dimension of $\mathcal{O}(\mathbf{L})$, the space of holomorphic sections of $\mathbf{L}$.

\section{A WEAK BUMPY METRIC THEOREM FOR HARMONIC SURFACES}

To illustrate the approach that we will use throughout the rest of the article, we now prove a bumpy metric theorem for harmonic maps which satisfy additional hypotheses.

Following 23, we say that a harmonic map $f: \Sigma \rightarrow M$ is somewhere injective if there is a point $p \in \Sigma$ such that $f^{-1}(f(p))=\{p\}$. The hypothesis, somewhere injective, is imposed to eliminate degenerate harmonic maps, such as torus parametrizations of geodesics, which would have to be treated in a complete theory of harmonic surfaces.

A map which is somewhere injective satisfies the condition $f^{-1}(f(p))=\{p\}$, for $p$ lying in an open dense set of points. To prove this, we can use a theorem of Sampson (33], Theorem 1) which states that two harmonic maps $f, f^{\prime}: D \rightarrow M$ from a connected domain $D$ in a Riemann surface which agree on an open set must be identical as maps. As in Yang-Mills Theory (Theorem 6.38 of [15]) and in the theory of $J$-holomorphic curves 23, the proof is an application of Aronszajn's unique continuation theorem [6] to the equation

$$
\frac{\partial^{2} u_{k}}{\partial x_{1}^{2}}+\frac{\partial^{2} u_{k}}{\partial x_{2}^{2}}+\sum_{i, j} \Gamma_{i j}^{k}\left(\frac{\partial u_{i}}{\partial x_{1}} \frac{\partial u_{j}}{\partial x_{1}}+\frac{\partial u_{i}}{\partial x_{2}} \frac{\partial u_{j}}{\partial x_{2}}\right)=0,
$$

for harmonic maps, with the abbreviation $u_{k}$ for $u_{k} \circ f$.

Proposition 3.1. Suppose that $M$ is a compact connected manifold of dimension at least three and $\omega \in \mathcal{T}_{g}$, the Teichmüller space for the Riemann surface of genus g. For a generic choice of Riemannian metric on $M$, every compact somewhere injective $\omega$-harmonic surface $f: \Sigma \rightarrow M$ with no branch points is as nondegenerate as allowed by its connected group $G$ of symmetries. If $G$ is trivial, it is Morse nondegenerate for $E_{\omega}$ in the usual sense, while if $G=S^{1} \times S^{1}$ or $P S L(2, \mathbb{C})$, it lies on a nondegenerate critical submanifold for $E_{\omega}$, which is an orbit for the $G$-action. If the genus of $\Sigma$ is at least one, the hypothesis that $f$ has no branch points can be weakened to the hypothesis that the space $\mathcal{O}(\mathbf{L})$ has the same real dimension as $G$.

The branch point assumption (or the assumption on $\mathcal{O}(\mathbf{L})$ ) is used to insure that the space of tangential Jacobi fields (isomorphic to the space of holomorphic sections of $\mathbf{L}$ ) is exactly the space generated by the action of $G$ :

(1) If $\Sigma$ is a two-sphere, the hypothesis $\nu=0$ is equivalent to $\operatorname{dim}_{\mathbb{C}} \mathcal{O}(\mathbf{L})=3$, the minimum possible value, as we explained in the previous section.

(2) If $\Sigma$ is a torus, $\operatorname{dim}_{\mathbb{C}} \mathcal{O}(\mathbf{L})=1$, the minimum possible value, exactly when $f$ has total branching order zero or one. 
(3) If $\Sigma$ has genus at least two, $\mathcal{O}(\mathbf{L})=0$ when the total branching order is $<2 g-2$, and if $\mathbf{L}$ is nontrivial as a holomorphic bundle when the total branching order is $2 g-2$.

The techniques for the proof of Proposition 3.1 are similar to those employed in 36] and 38. In our argument, we will utilize the space

$\operatorname{Map}^{\prime}(\Sigma, M)=\{f \in \operatorname{Map}(\Sigma, M)$ such that $f$ is somewhere injective $\}$,

as well as

$$
\operatorname{Met}(M)=\{\text { Riemannian metrics on } M\} \text {, }
$$

which we assume have been completed with respect to a Sobolev $L_{k}^{2}$ norm for a sufficiently large value of $k$. When it is necessary to be specific about the value of $k$, we will write

$$
\left(L_{k}^{2}\right)^{\prime}(\Sigma, M), \quad L_{k}^{2}(\Sigma, M) \text { and } \operatorname{Met}(M)_{k}^{2}
$$

instead of

$$
\operatorname{Map}^{\prime}(\Sigma, M), \quad \operatorname{Map}(\Sigma, M) \text { and } \operatorname{Met}(M) .
$$

An important role will be played by the space of "forced Jacobi fields". If $\operatorname{Map}(\Sigma, M)$ is given a sufficiently strong topology, these are the pushforwards of vector fields on $\Sigma$ generated by the group $G$ of symmetries acting on the domain $\Sigma$ and are sections of $\left(f^{*} T M\right)^{\top}$. These fields generate a finite-dimensional subbundle $\mathcal{F}$ of the restriction of the bundle

$$
T \operatorname{Map}(\Sigma, M): \operatorname{Map}(\Sigma, T M) \longrightarrow \operatorname{Map}(\Sigma, M)
$$

to $\operatorname{Map}^{\prime}(\Sigma, M)$, in which the projection is induced by the projection on the range $\pi: T M \rightarrow M$ by means of the $\omega$-Lemma, the fiber of the bundle (17) being the space of sections of $f^{*} T M$. The finite-dimensional subbundle $\mathcal{F}$ has an $L^{2}$ orthogonal complement which we denote by $\mathcal{E}$.

We call elements of the subbundle $\mathcal{F}$ forced Jacobi fields even though when $f$ is not a harmonic map, the sections of the fiber $\mathcal{F}_{f}$ are not Jacobi fields in the usual sense, because $f$ is not a critical point for $E_{\omega}$. We emphasize that if $f$ is harmonic, the elements of the fiber $\mathcal{F}_{f}$ are the real and imaginary parts of elements of $\mathcal{O}(\mathbf{L})$. We denote the real rank of the bundle $\mathcal{F}$ by $d_{\Sigma}$ :

- $d_{\Sigma}=6$, when $\Sigma=S^{2}$,

- $d_{\Sigma}=2$, when $\Sigma=T^{2}$,

- $d_{\Sigma}=0$, when $\Sigma$ has genus greater than one.

We let

$$
\begin{aligned}
\mathcal{S}_{\omega}^{\prime}=\left\{(f, g) \in \operatorname{Map}^{\prime}(\Sigma, M) \times \operatorname{Met}(M): f\right. \text { is somewhere injective, } \\
\left.\omega \text {-harmonic for } g \text { and } 2 \operatorname{dim}_{\mathbb{C}} \mathcal{O}(L)=d_{\Sigma}\right\} .
\end{aligned}
$$

The overall strategy of the proof is to show that $\mathcal{S}_{\omega}^{\prime}$ is a submanifold of the product $\operatorname{Map}^{\prime}(\Sigma, M) \times \operatorname{Met}(M)$ and that the projection $\pi: \mathcal{S}_{\omega}^{\prime} \rightarrow \operatorname{Met}(M)$ is Fredholm of Fredholm index $d_{\Sigma}$.

To carry out this strategy, we first consider the smooth Euler-Lagrange map

$$
F:\left(L_{k}^{2}\right)^{\prime}(\Sigma, M) \times \operatorname{Met}(M)_{k-1}^{2} \longrightarrow L_{k-2}^{2}(\Sigma, T M)
$$

defined in terms of local conformal coordinates $\left(x^{1}, x^{2}\right)$ on $\Sigma$, by

$$
F(f, g)=-\frac{1}{\lambda^{2}}\left[\frac{D^{g}}{\partial x_{1}}\left(\frac{\partial f}{\partial x_{1}}\right)+\frac{D^{g}}{\partial x_{2}}\left(\frac{\partial f}{\partial x_{2}}\right)\right],
$$




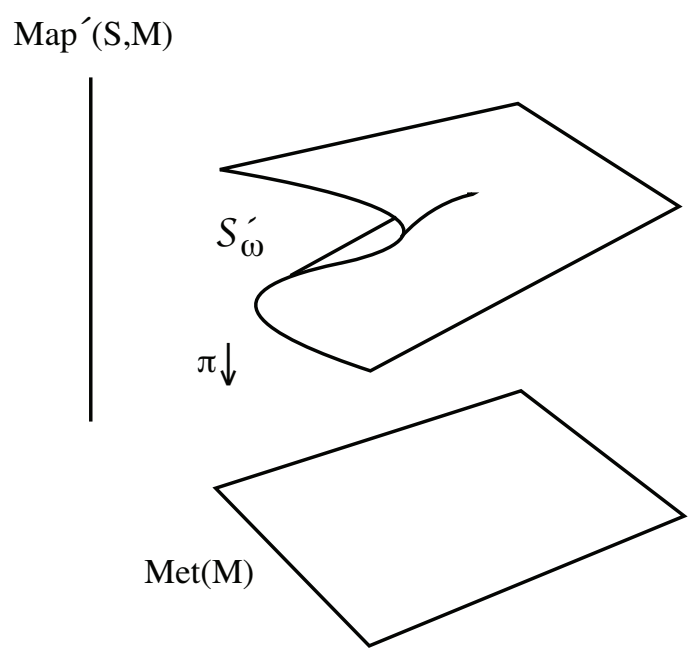

Figure 1. The overall strategy of the proof is to show that $\mathcal{S}_{\omega}^{\prime}$ is a submanifold and the projection $\pi: \mathcal{S}_{\omega}^{\prime} \rightarrow \operatorname{Met}(M)$ is Fredholm of Fredholm index $d_{\Sigma}$.

where $\lambda^{2}\left(d x_{1}^{2}+d x_{2}^{2}\right)$ is the canonical Riemannian metric on $\Sigma$ and $D^{g}$ is the covariant derivative for the Levi-Civita connection on $T M$ determined by the Riemannian metric $g$ on $M$. Note that $\pi \circ F(f, g)=f$, where $\pi$ denotes the projection of the pullback of the bundle

$$
\pi: L_{k-2}^{2}(\Sigma, T M) \rightarrow L_{k-2}^{2}(\Sigma, M)
$$

to $L_{k}^{2}(\Sigma, M)$. Let $p: \operatorname{Map}(\Sigma, T M) \longrightarrow \mathcal{E}$ denote the bundle map which restricts on each fiber to the orthogonal projection onto the $L^{2}$-orthogonal complement of the forced Jacobi fields, and let $\mathcal{Z}$ be the image of the zero section in $\mathcal{E}$. Our goal is to show that $p \circ F$ intersects $\mathcal{Z}$ transversally.

By the first variation formula (36),

$$
\int_{\Sigma}\langle F(f, g), X\rangle d A=d E_{\omega}(f)(X)=0, \quad \text { when } \quad X \in \mathcal{F}_{f}
$$

that is, when $X$ is a vector field generated by the action of $G$, so the zero set of $p \circ F$ is the same as that of $F$. Thus once we know that $p \circ F$ intersects $\mathcal{Z}$ transversally, we will have established that

$$
\mathcal{S}_{\omega}^{\prime}=F^{-1}(\mathcal{Z})=(p \circ F)^{-1}(\mathcal{Z})
$$

is a submanifold.

Note that at the image of a zero $(f, g)$ for $F$, the tangent spaces to the total spaces of our vector bundles $\mathcal{E}$ or $T \operatorname{Map}^{\prime}(\Sigma, M)$ can be divided into direct sums

$$
T_{0_{f}} \mathcal{E}=H \oplus V, \quad T_{0_{f}}\left(\operatorname{Map}^{\prime}(\Sigma, M)\right)=H \oplus V,
$$

where $0_{f}$ denotes the zero section located at $f, H$ is the horizontal space (tangent to the space of zero sections) and $V$ is the vertical space (tangent to the fiber). Let $\pi_{V}$ denote the projection onto $V$ along $H$. To prove transversality, it will suffice to 
show that $\mathcal{V}=0$, where

$$
\begin{aligned}
\mathcal{V}=\left\{L_{k-2}^{2} \text {-sections } X\right. & \text { of } \mathcal{E}_{0_{f}}: X \text { is perpendicular to } \\
& \text { the image of } \left.\pi_{V} \circ D(p \circ F)_{(f, g)}=p \circ \pi_{V} \circ D F_{(f, g)}\right\},
\end{aligned}
$$

perpendicular being with respect to the $L^{2}$ inner product. Note that it follows from (6) that

$$
p \circ \pi_{V} \circ\left(D_{1} F\right)_{(f, g)}(X)=p \circ L(X),
$$

where $L=L_{(f, g)}$ is the Jacobi operator. The other component

$$
p \circ \pi_{V} \circ\left(D_{2} F\right)_{(f, g)}(\dot{g}),
$$

where $D_{2} F$ is the partial derivative with respect to the second variable $g \in \operatorname{Met}(M)$, gives the effect of deformations in the metric on $M$.

For later reference, we note that the following lemma holds for somewhere injective critical points $f$ for $E_{\omega}$, whether they have branch points or not.

Lemma 3.1. If $f$ is a somewhere injective critical point for $E_{\omega}$, the only sections of $f^{*} T M$ which can be perpendicular to the image of every variation in the metric are those which are real and imaginary parts of holomorphic sections of $\mathbf{L}$.

Proof. To prove this, we need to calculate

$$
p \circ \pi_{V} \circ\left(D_{2} F\right)_{(f, g)}(\dot{g}),
$$

at an element $(f, g) \in \mathcal{S}_{\omega}^{\prime}$ for various choices of the deformation $\dot{g} \in T_{g} \operatorname{Met}(M)$. Note that since $F(f, g)$ is the zero section,

$$
\left\langle p \circ \pi_{V} \circ\left(D_{2} F\right), X\right\rangle=D_{2}\langle p \circ F(f, g), X\rangle .
$$

In this equation, $\langle\cdot, \cdot\rangle$ is another name for the metric $g$ on the ambient space $M$.

Suppose that $f$ is an $\omega$-harmonic map for $g$. The set $W \subset \Sigma$ which consists of points $p$ such that $p$ is not a branch point and $f^{-1}(f(p))=p$ is open and dense by hypothesis. Let us choose an open neighborhood $U$ of a point $p \in W$ such that $U \subset W$ and $f$ imbeds $U$ onto $f(\Sigma) \cap V$ for some open set $V \subset M$. Arrange, moreover, that $V$ is the domain of local coordinates $\left(u_{1}, \ldots, u_{n}\right)$ such that $u_{i}(f(p))=0$ and

(1) $f(U)$ is described by the equations $u_{3}=\cdots=u_{n}=0$,

(2) $u_{a} \circ f=x_{a}$ on $f(U)$, for $a=1,2$, where $x_{1}+i x_{2}$ is a conformal parameter on $U$, and

(3) the Riemannian metric $g$ on the ambient space takes the form $\sum g_{i j} d u_{i} d u_{j}$, such that when restricted to $f(\Sigma) \cap V, g_{i r}=\delta_{i r}$, for $1 \leq i \leq n$ and $3 \leq r \leq n$.

Such coordinates can be constructed using the exponential map restricted to the normal bundle of the surface $f(\Sigma) \cap V$ in $M$.

Recall that in terms of local coordinates, the equation for harmonic maps can be written as

$$
\frac{1}{\lambda^{2}}\left(\frac{\partial^{2} u_{k}}{\partial x_{1}^{2}}+\frac{\partial^{2} u_{k}}{\partial x_{2}^{2}}\right)+\sum_{i, j} \frac{1}{\lambda^{2}} \Gamma_{i j}^{k}\left(\frac{\partial u_{i}}{\partial x_{1}} \frac{\partial u_{j}}{\partial x_{1}}+\frac{\partial u_{i}}{\partial x_{2}} \frac{\partial u_{j}}{\partial x_{2}}\right)=0
$$

the canonical constant curvature Riemannian metric on $\Sigma$ being $\lambda^{2}\left(d x_{1}^{2}+d x_{2}^{2}\right)$. The expression on the left-hand side of this equation is often called the tension of the 
map $f$, a harmonic map being one which has zero tension. In terms of the special local coordinates we have chosen, the equation for harmonic maps yields

$$
\Gamma_{11}^{k}+\Gamma_{22}^{k}=0 .
$$

A perturbation in the metric $\dot{g} \in T_{g} \operatorname{Met}(M)$ with compact support in $V$ can be written in the form $\dot{g}=\sum \dot{g}_{i j} d x_{i} d x_{j}$, where the $\dot{g}_{i j}$ 's are smooth functions on $V$. Under such a perturbation, the only part of the tension that changes is the Christoffel symbol

$$
\Gamma_{i j}^{k}=\sum g^{k l} \Gamma_{l, i j}, \quad \text { where } \quad \Gamma_{l, i j}=\frac{1}{2}\left(\frac{\partial g_{i l}}{\partial u_{j}}+\frac{\partial g_{j l}}{\partial u_{i}}-\frac{\partial g_{i j}}{\partial u_{l}}\right) .
$$

If $\dot{\Gamma}_{k, i j}$ denotes the derivative of $\Gamma_{k, i j}$ in the direction of the perturbation,

$$
\dot{\Gamma}_{k, i j}=\frac{1}{2}\left(\frac{\partial \dot{g}_{i k}}{\partial u_{j}}+\frac{\partial \dot{g}_{j k}}{\partial u_{i}}-\frac{\partial \dot{g}_{i j}}{\partial u_{k}}\right) .
$$

From this formula, we can calculate that

$$
\begin{aligned}
D_{2}\langle F(f, g), X\rangle(\dot{g}) & =-\frac{1}{\lambda^{2}} \sum_{i, j, k=1}^{n} \dot{\Gamma}_{k, i j}\left(\frac{\partial u_{i}}{\partial x_{1}} \frac{\partial u_{j}}{\partial x_{1}}+\frac{\partial u_{i}}{\partial x_{2}} \frac{\partial u_{j}}{\partial x_{2}}\right) f^{k} \\
& =-\frac{1}{\lambda^{2}} \sum_{k=1}^{n}\left(\dot{\Gamma}_{k, 11}+\dot{\Gamma}_{k, 22}\right) f^{k}, \quad \text { when } \quad X=\sum_{i=1}^{n} f^{i} \frac{\partial}{\partial u_{i}}
\end{aligned}
$$

is a fixed vector field along $f$. Thus the definition (19) of $\mathcal{V}$ implies that

$$
X \in \mathcal{V} \quad \Rightarrow \quad \int_{\Sigma} \sum_{k=1}^{n} \sum_{a=1}^{2} f^{k} \dot{\Gamma}_{k, a a} d x_{1} d x_{2}=0
$$

for all perturbations in the metric.

If we set

$$
\dot{g}_{11}\left(u_{1}, \ldots, u_{n}\right)=\dot{g}_{22}\left(u_{1}, \ldots, u_{n}\right)=\sum_{r=3}^{n} u_{r} \phi_{r}\left(u_{1}, u_{2}\right),
$$

and let $\dot{g}_{i j}=0$ for the other choices of indices $i, j$, a straightforward calculation shows that the fiber projection of the partial derivative of $F$ with respect to $g$ is given by the expression

$$
\pi_{V} \circ\left(D_{2} F\right)_{(f, g)}(\dot{g})=\frac{1}{2 \lambda^{2}} \sum_{r=3}^{n} \sum_{a=1}^{2} \frac{\partial \dot{g}_{a a}}{\partial u_{r}} \frac{\partial}{\partial u_{r}}=\frac{1}{\lambda^{2}} \sum_{r=3}^{n} \frac{\partial \dot{g}_{11}}{\partial u_{r}} \frac{\partial}{\partial u_{r}} .
$$

Thus we see that any vector field of the form

$$
\sum_{r=3}^{n} \phi_{r}\left(u_{1}, u_{2}\right) \frac{\partial}{\partial u_{r}}
$$

where $\phi_{r}$ is a smooth function on $U$ with compact support, lies in the image of $\pi_{V} \circ\left(D_{2} F\right)_{(f, g)}$, and hence elements of $\mathcal{V} \otimes \mathbb{C}$ must restrict to sections of $\mathbf{L} \oplus \overline{\mathbf{L}}$ over $U$. Since a dense open subset of points of $\Sigma$ can be covered by sets of the form $U$, we see that elements of $\mathcal{V}$ must be sections of $\mathbf{L} \oplus \overline{\mathbf{L}}$ over all of $\Sigma$.

To determine the conditions satisfied by the tangential components of elements of $\mathcal{V}$, we set

$$
\dot{g}_{11}=-\dot{g}_{22}=M, \quad \dot{g}_{12}=N, \quad \dot{g}_{i j}=0 \quad \text { for other choices of indices } i, j,
$$


where $M$ and $N$ have compact support within $V$, and a short calculation shows that

$$
\begin{aligned}
& \dot{\Gamma}_{1,11}+\dot{\Gamma}_{1,22}=\frac{1}{2} \frac{\partial \dot{g}_{11}}{\partial u_{1}}+\frac{\partial \dot{g}_{12}}{\partial u_{2}}-\frac{1}{2} \frac{\partial \dot{g}_{22}}{\partial u_{1}}=\frac{\partial M}{\partial u_{1}}+\frac{\partial N}{\partial u_{2}} \\
& \dot{\Gamma}_{2,11}+\dot{\Gamma}_{2,22}=\frac{\partial \dot{g}_{12}}{\partial u_{1}}-\frac{1}{2} \frac{\partial \dot{g}_{11}}{\partial u_{2}}+\frac{1}{2} \frac{\partial \dot{g}_{22}}{\partial u_{2}}=\frac{\partial N}{\partial u_{1}}-\frac{\partial M}{\partial u_{2}}
\end{aligned}
$$

Hence in this case,

$$
\begin{aligned}
X=\sum_{a=1}^{2} f^{a} \frac{\partial}{\partial u_{a}}=\sum_{a=1}^{2} f^{a} \frac{\partial}{\partial x_{a}} \Rightarrow \\
\quad D_{2}\langle F(f, g), X\rangle(\dot{g})=-\frac{1}{\lambda^{2}}\left[\left(\frac{\partial M}{\partial x_{1}}+\frac{\partial N}{\partial x_{2}}\right) f^{1}+\left(\frac{\partial N}{\partial x_{1}}-\frac{\partial M}{\partial x_{2}}\right) f^{2}\right]
\end{aligned}
$$

and

$$
\begin{aligned}
\int_{\Sigma}\left\langle\pi_{V} \circ\left(D_{2} F\right)_{(f, g)}(\dot{g}), X\right\rangle d A & \\
= & -\int_{\Sigma}\left[\left(\frac{\partial M}{\partial x_{1}}+\frac{\partial N}{\partial x_{2}}\right) f^{1}+\left(\frac{\partial N}{\partial x_{1}}-\frac{\partial M}{\partial x_{2}}\right) f^{2}\right] d x_{1} d x_{2} .
\end{aligned}
$$

In particular, $f^{1}\left(\partial / \partial x_{1}\right)+f^{2}\left(\partial / \partial x_{2}\right)$ is perpendicular to the range of the map $\pi_{V} \circ\left(D_{2} F\right)_{(f, g)}$ if and only if

$$
\int_{\Sigma}\left[f^{1}\left(\frac{\partial M}{\partial x_{1}}+\frac{\partial N}{\partial x_{2}}\right)+f^{2}\left(\frac{\partial N}{\partial x_{1}}-\frac{\partial M}{\partial x_{2}}\right)\right] d x_{1} d x_{2}=0
$$

or equivalently, if and only if

$$
\int_{\Sigma}\left[M\left(\frac{\partial f^{1}}{\partial x_{1}}-\frac{\partial f^{2}}{\partial x_{2}}\right)+N\left(\frac{\partial f^{1}}{\partial x_{2}}+\frac{\partial f^{2}}{\partial x_{1}}\right)\right] d x_{1} d x_{2}=0
$$

for all choices of functions $M$ and $N$, which one verifies is exactly the condition that $f^{1}\left(\partial / \partial x_{1}\right)+f^{2}\left(\partial / \partial x_{2}\right)$ be the real or imaginary part of a holomorphic section of $\mathbf{L}$. It follows that the $L^{2}$ orthogonal complement of the range of $\pi_{V} \circ\left(D_{2} F\right)_{(f, g)}$ is contained in the space of real and imaginary parts of holomorphic sections of $\mathbf{L}$, and the lemma is proven.

Under the hypothesis on the dimension of $\mathcal{O}(\mathbf{L})$ stated in Proposition 3.1, the real and imaginary parts of holomorphic sections of $\mathbf{L}$ are forced Jacobi fields, and hence do not lie within the fiber of $\mathcal{E}$. It therefore follows from Lemma 3.1 that $\mathcal{V}=0$ and $\mathcal{S}_{\omega}^{\prime}$ is a smooth submanifold of $\operatorname{Map}^{\prime}(\Sigma, M) \times \operatorname{Met}(M)$. Note that under the hypotheses of Proposition 3.1, $p \circ \pi_{V} \circ\left(D_{2} F\right)_{(f, g)}(\dot{g})$ is surjective, a fact which is useful in applications.

Remark 3.1. For later steps in the proof of the Main Theorem, it will be important to recognize that the tangential variation $\dot{g}$ that we have used in the second part of the above proof can be regarded as defined by a quadratic differential $\dot{\omega}=h d z^{2}=$ $(M-i N) d z^{2}$. If

$$
Z=\left(f^{1}+i f^{2}\right)\left(\frac{\partial}{\partial x_{1}}-i \frac{\partial}{\partial x_{2}}\right)=2\left(f^{1}+i f^{2}\right) \frac{\partial}{\partial z},
$$


so that the real part of $Z$ is the vector field $X$ considered above, then (23) yields

$$
D_{2}\langle F(f, g), Z\rangle(\dot{g})=-\frac{2}{\lambda^{2}}\left(\left(f^{1}+i f^{2}\right) \frac{\partial}{\partial \bar{z}}(M-i N)\right)=-\frac{1}{\lambda^{2}} \frac{\partial h}{\partial \bar{z}} d z(Z),
$$

where we have extended $\langle F(f, g), \cdot\rangle$ to be complex linear. We set

$$
B(\dot{\omega}, Z)=\int_{\Sigma} D_{2}\langle F(f, g), Z\rangle(\dot{g}) d A,
$$

a complex bilinear map that will be used later. In view of the fact that

$$
d A=\lambda^{2} d x \wedge d y=\frac{i}{2} \lambda^{2} d z \wedge d \bar{z}
$$

the above calculations show that

$$
B(\dot{\omega}, Z)=-\frac{i}{2} \int_{\Sigma}\left(\frac{\partial h}{\partial \bar{z}} d z(Z)\right) d z \wedge d \bar{z}=-\frac{i}{2} \int_{\Sigma} \bar{\partial}(\dot{\omega})(Z) .
$$

Here $\bar{\partial}$ is the usual first-order differential operator from $\mathbf{K}^{2}$ to $\mathbf{K}^{2} \otimes \overline{\mathbf{K}}$, which contracts with $\mathbf{K}^{-1}$ to yield an integrand, an element of $\mathbf{K} \otimes \overline{\mathbf{K}}$.

An integration by parts yields

$$
\begin{aligned}
\int_{\Sigma}\left\langle\pi_{V} \circ\left(D_{2} F\right)_{(f, g)}(\dot{g}), Z\right\rangle d A=-\int_{\Sigma} \frac{1}{\lambda^{2}}\left(\frac{\partial h}{\partial \bar{z}} d z(Z)\right) d A & \\
& =\int_{\Sigma} \frac{1}{\lambda^{2}} h d z\left(\frac{D Z}{\partial \bar{z}}\right) d x_{1} d x_{2} .
\end{aligned}
$$

If $Z$ is a section of $\mathbf{L}$ it can be written in terms of a local complex parameter $z$ in the form $Z=\zeta(\partial f / \partial z)$, where $\zeta$ is a complex-valued function. In the case where $f$ is conformal, say $f^{*}\langle\cdot, \cdot\rangle=\sigma^{2}\left(d x_{1}^{2}+d x_{2}^{2}\right)$, we can write

$$
\left\langle\frac{\partial f}{\partial \bar{z}}, \frac{\partial f}{\partial z}\right\rangle=\frac{\sigma^{2}}{2} \quad \Rightarrow \quad d z\left(\frac{D Z}{\partial \bar{z}}\right)=\frac{\partial \zeta}{\partial \bar{z}}=\frac{2}{\sigma^{2}}\left\langle\frac{\partial f}{\partial \bar{z}}, \frac{D Z}{\partial \bar{z}}\right\rangle .
$$

In this case, comparison with (27) yields a formula which will be needed in 6 .

$$
\int_{\Sigma}\left\langle\pi_{V} \circ\left(D_{2} F\right)_{(f, g)}(\dot{g}), Z\right\rangle d A=\int_{\Sigma} \frac{2}{\lambda^{2}}\left\langle\frac{D Z}{\partial \bar{z}}, \frac{h}{\sigma^{2}} \frac{\partial f}{\partial \bar{z}}\right\rangle d A .
$$

Lemma 3.2. The projection on the second factor $\pi: \mathcal{S}_{\omega}^{\prime} \rightarrow \operatorname{Met}(M)$ is a Fredholm map of Fredholm index $d_{\Sigma}$, the real dimension of the symmetry group $G$.

Proof. We first need to determine the tangent space to $\mathcal{S}_{\omega}^{\prime}$ at $(f, g)$. This is a straightforward calculation which ends with the result:

$$
\begin{aligned}
T_{(f, g)} \mathcal{S}_{\omega}^{\prime}=\left\{(X, h) \in T_{f} \operatorname{Map}(\Sigma, M) \oplus\right. & T_{g} \operatorname{Met}(M): \\
& \left.p \circ L(X)+\pi_{V} \circ\left(D_{2} F\right)_{(f, g)}(h)=0\right\},
\end{aligned}
$$

where $L=L_{(f, g)}$ is the Jacobi operator at $(f, g)$ and $p$ is the projection into the bundle $\mathcal{E}$ considered earlier in this section. Thus $p$ is the projection onto the $L^{2}$ orthogonal complement of the space $\mathcal{F}$ of forced Jacobi fields, the vector fields generated by the $G$-action. We can calculate the kernel and the range of the map

$$
d \pi_{(f, g)}: T_{(f, g)} \mathcal{S}_{\omega}^{\prime} \rightarrow T_{g} \operatorname{Met}(M)
$$


and we find that

Kernel of $d \pi_{(f, g)}=\left\{(X, h) \in T_{f} \operatorname{Map}(\Sigma, M) \oplus T_{g} \operatorname{Met}(M):\right.$

$$
h=0, p \circ L(X)=0\},
$$

while

Range of $d \pi_{(f, g)}=\left\{h \in T_{g} \operatorname{Met}(M): \pi_{V} \circ\left(D_{2} F\right)_{(f, g)}(h)+p \circ L(X)=0\right.$, for some $\left.X \in T_{f} \operatorname{Map}(\Sigma, M)\right\}$.

Recall that the Jacobi operator

$$
L=L_{(f, g)}:\left(L_{k}^{2} \text { sections of } f^{*} T M\right) \longrightarrow\left(L_{k-2}^{2} \text { sections of } f^{*} T M\right)
$$

is Fredholm, and since it is formally self-adjoint it has Fredholm index zero, when $k \geq 2$. (One can take $k=2$ for the sake of this proof.) Moreover, since $L$ is equivariant with respect to the $G$-actions on both domain and range, both the kernel of $L$ and the orthogonal complement to its range contain a $d_{\Sigma}$-dimensional subspace generated by the $G$-action.

Clearly, the kernel of $d \pi_{(f, g)}$ is isomorphic to the kernel of $L$ while it is easily verified that the $L^{2}$ orthogonal complement to the range is simply the space of metric deformations $h$ such that $\pi_{V} \circ\left(D_{2} F\right)_{(f, g)}(h)$ is a Jacobi field. It therefore follows from Lemma 3.1 that the range of $d \pi_{(f, g)}$ is closed and its codimension is the dimension of the space of Jacobi fields orthogonal to $\mathcal{F}$. Since the dimension of the kernel of $d \pi_{(f, g)}$ is the dimension of the space of Jacobi fields, we find that the Fredholm index of $d \pi_{(f, g)}$ is $d_{\Sigma}$, the dimension of $\mathcal{F}$, finishing the proof of Lemma 3.2 .

Finally, we use the Sard-Smale Theorem 35 to conclude that a generic subset of $\operatorname{Met}(M)$ consists of regular values for the projection $\pi$ on the second factor. If $g_{0}$ is a regular value of the projection, $\pi^{-1}\left(g_{0}\right)$ consists of connected $d_{\Sigma}$-dimensional nondegenerate critical submanifolds, manifolds of exactly the dimension forced by the symmetry. These submanifolds must be $G$-orbits of critical points. This finishes the proof of Proposition 3.1.

Remark 3.2. The argument presented in this section lacks a certain elegance in that the map $F$ we employed loses two derivatives. However, we can easily rephrase the argument in terms of a map that involves no loss of derivatives.

To do this, we first define a weak Riemannian metric $\langle\langle\cdot, \cdot\rangle\rangle_{1}$ on $L_{k}^{2}(\Sigma, M)$ by setting

$$
\langle\langle X, Y\rangle\rangle_{1}=\int_{\Sigma}[\langle D X, D Y\rangle+\langle X, Y\rangle] d A, \quad \text { for } X, Y \text { sections of } f^{*} T M,
$$

where $D X$ is the covariant differential of the section $X$ of $f^{*} T M$ and $\langle D X, D Y\rangle$ is calculated with respect to the Riemannian metrics on both $\Sigma$ and $M$. We can then define a Green's operator

$$
B:\left(L_{k-2}^{2} \text {-sections of } f^{*} T M\right) \rightarrow\left(L_{k}^{2} \text {-sections of } f^{*} T M\right)
$$

by

$$
\langle\langle B(X), Y\rangle\rangle_{1}=\int_{\Sigma}\langle X, Y\rangle d A .
$$


Note that $B$ is the inverse of an elliptic operator which does not contain zero in its spectrum, and hence is a linear topological vector space isomorphism. We now consider the composition

$$
A=B \circ F:\left(L_{k}^{2}\right)^{\prime}(\Sigma, M) \times \operatorname{Met}(M)_{k-1}^{2} \longrightarrow L_{k}^{2}(\Sigma, T M),
$$

which does not lose derivatives. If $f$ is an $\omega$-harmonic map, $\pi_{V} \circ F(f)=0$, so $D\left(\pi_{V} \circ A\right)=B \circ D\left(\pi_{V} \circ F\right)$ and we can rephrase the proofs of Lemmas 3.1 and 3.2 in terms of $A$. Moreover, if $f$ is an $\omega$-harmonic map,

$$
d^{2} E_{\omega}(f)(X, Y)=\langle\langle A(X), Y\rangle\rangle_{1}, \quad \text { whenever } X \text { and } Y \text { are sections of } f^{*} T M .
$$

Finally, we point out that the operator $A$ can be chosen to commute with inclusions

$$
\left\{L_{k}^{2} \text {-sections of } f^{*} T M\right\} \subset\left\{L_{k-1}^{2} \text {-sections of } f^{*} T M\right\} \text {. }
$$

\section{Self-intersections}

In this section, we will show that prime parametrized minimal surfaces are automatically somewhere injective. We will need this fact to generalize the argument from the preceding section to arbitrary prime parametrized minimal surfaces.

In fact, we will need a much more explicit description of the set of self-intersections, and for this we need to understand the behavior of minimal surfaces near branch points. Recall that in terms of a local holomorphic coordinate $z$ centered at a branch point of order $\nu$, we can write $(\partial f / \partial z)(z)=z^{\nu} g(z)$, where $g$ is a holomorphic section with $g(0) \neq 0$, and it follows directly from Taylor's theorem that in terms of suitable normal coordinates $\left(u_{1}, \ldots, u_{n}\right)$ centered at $f(p)$, we can write

$$
\left(u_{1}+i u_{2}\right)(z)=c z^{\nu+1}+o_{1}\left(z^{\nu+1}\right), \quad u_{r}(z)=o_{1}\left(z^{\nu+1}\right) \quad \text { for } \quad 3 \leq r \leq n,
$$

$c$ being a nonzero complex constant. Here $o_{1}\left(z^{k+1}\right)$ stands for a term which is $o\left(z^{k+1}\right)$, and which has a derivative that is $o\left(z^{k}\right)$.

Much stronger results can be established. Indeed, it follows from Theorem 1.4 of Micallef and White 27. that if $p$ is a branch point of a weakly conformal harmonic map $f: \Sigma \rightarrow M$, then there are normal coordinates $\left(u_{1}, \ldots, u_{n}\right)$ centered at $f(p)$ on the ambient Riemannian manifold $M$ and a $C^{2, \alpha}$ diffeomorphism $z \mapsto \xi(z)$ near $p$ on $\Sigma, z$ being a holomorphic coordinate centered at $p$, such that $\xi(z)=c z+O\left(|z|^{2}\right)$ for some nonzero complex constant $c$, and if we abbreviate the composition $u_{i} \circ f \circ \xi$ to $u_{i}$, then

$$
u_{1}(z)+i u_{2}(z)=\xi(z)^{\nu+1}, \quad u_{r}(z)=f_{r}(\xi(z)) \quad \text { for } \quad 3 \leq r \leq n,
$$

where each $f_{r}(\xi)$ is $o_{1}\left(|\xi|^{\nu+1}\right)$. Thus in terms of the nonconformal coordinate $\xi$, the error term in $u_{1}+i u_{2}$ vanishes. If we use $\xi$ as the local (nonconformal) parameter on $\Sigma$, we can write (31) as

$$
\xi \mapsto\left(\xi^{\nu+1}, \tilde{f}(\xi)\right), \quad \text { where } \tilde{f} \text { takes values in } \mathbb{R}^{n-2} .
$$

Theorem 1.4 of [27] goes on to state that if $\mu^{\nu+1}=1$, then either $\tilde{f}(\mu \xi)-\tilde{f}(\xi)$ is identically zero, or

$$
\tilde{f}(\mu \xi)-\tilde{f}(\xi)=a \xi^{\rho}+\bar{a} \bar{\xi}^{\rho}+o_{1}\left(|\xi|^{\rho}\right),
$$

where $a$ is a nonzero element of $\mathbb{C}^{n-2}$ and $\rho>\nu+1$.

We say that a branch point $p$ for a harmonic map $f$ is ramified of order $\sigma$ if $\sigma+1$ is the largest integer such that there is an open neighborhood $U$ of $p$ and a smooth 
map $\psi: U \rightarrow D$, where $D$ is the unit disk centered at 0 in the complex plane, such that

(1) $\psi(p)=0$

(2) $\psi \mid(U-\{p\})$ is a $(\sigma+1)$-sheeted cover of $D-\{0\}$, and

(3) if $q, r \in U-\{p\}$, then $f(q)=f(r) \Leftrightarrow \psi(q)=\psi(r)$.

If $\sigma=\nu$, the branching order of $p$, we say that $p$ is a false branch point. At the other extreme, we say that the branch point is primitive or unramified if the largest such integer $\sigma$ is zero.

Note that a branch point is primitive precisely when $\tilde{f}(\mu \xi)-\tilde{f}(\xi)$ is not identically zero for any nontrivial $(\nu+1)$-fold root of unity.

Lemma 4.1. If $f: \Sigma \rightarrow M$ is a prime parametrized minimal surface, all its branch points are primitive.

This follows quickly from the Aronszajn unique continuation theorem and the theory of branched immersions presented by Gulliver, Osserman and Royden in $\S 3$ of [17. For the convenience of the reader we sketch the idea behind the argument, referring to [17] for a detailed presentation.

We begin by defining an equivalence relation $\sim$ on points of $\Sigma$ by setting $p \sim q$ if there are open neighborhoods $U_{p}$ and $U_{q}$ of $p$ and $q$ respectively, and a conformal or anti-conformal diffeomorphism $\psi: U_{p} \rightarrow U_{q}$ such that $f \circ \psi=f$. (We make a minor modification to the construction in $\S 3$ of [17] by allowing $\psi$ to be orientationreversing so that we can allow for branched covers of nonorientable surfaces.) Using the argument in [17, which is based upon Aronsjazn's unique continuation theorem, one shows that $\sim$ is indeed an equivalence relation and that the quotient space $\Sigma_{0}$ is a topological manifold (possibly nonorientable) which is smooth except at the branch points. We can define $f_{0}: \Sigma_{0} \rightarrow M$ by $f_{0}([p])=f(p)$, where $[p]$ denotes the equivalence class of $p$, so that if $\pi: \Sigma \rightarrow \Sigma_{0}$ is the quotient map, $f_{0} \circ \pi=f$. We note that any point equivalent to a branch point is itself a branch point. The restriction of $f_{0}$ to $\Sigma_{0}$ minus the equivalence classes of the branch points is a harmonic map of finite energy. It therefore follows from the removeable singularity theorem of Sacks and Uhlenbeck (Theorem 3.6 of [31]) that the restriction of $f_{0}$ can be extended to the equivalence classes of the branch points so as to be a harmonic map, which is conformal so long as $f$ is conformal.

Thus $f$ is a branched cover of $f_{0}$. The hypothesis that $f$ is prime implies that $\Sigma_{0}$ must equal $\Sigma, \pi$ is the identity, and every branch point must be primitive, finishing our sketch of the proof.

A description of the intersection of two minimal surfaces was an important ingredient in the highly successful applications of minimal surface theory to the topology of three-manifolds developed by Meeks and Yau and others (see [25] and [16] for example), and we claim that a similar description can be established in the case in which the ambient space $M$ has arbitrary dimension $\geq 3$, using Lemma 4.1, the canonical form in a neighborhood of a branch point, and the following lemma, a special case of Lemma 2.4 of Cheng [11].

Lemma 4.2. Suppose that $f$ is a smooth real-valued function on an open neighborhood of the origin in $\mathbb{R}^{2}$ and $p$ is a nonzero homogeneous harmonic polynomial of degree $\rho \geq 2$ on $\mathbb{R}^{2}$ such that

$$
f(\xi)-p(\xi)=o_{1}\left(\left.\xi\right|^{\rho}\right), \quad \text { for some positive integer } \rho .
$$


Then there is a local $C^{1}$ diffeomorphism $\Phi$ of $\mathbb{R}^{2}$, fixing the origin, such that $f(\xi)=$ $p(\Phi(\xi))$.

We refer to [11], pages 48 and 49, for an elegant proof of this lemma.

With Lemmas 4.1 and 4.2 at our disposal, we are ready to present the main result of this section.

Lemma 4.3. Suppose that $f: \Sigma \rightarrow M$ is a prime compact connected parametrized minimal surface in a compact Riemannian manifold of dimension at least three. Let

$$
K=\left\{p \in \Sigma: f^{-1}(f(p)) \text { contains more than one point }\right\},
$$

a compact subset of $\Sigma$. Then each point within the self-intersection set $K$ belongs to one of two classes:

(1) an isolated point of self-intersection (of which there can be only finitely many),

(2) a point which lies in a neighborhood $U \subset \Sigma$ such that $K \cap U$ is a subset of a finite number of $C^{1}$ curve segments of finite length in $U$ intersecting at the point.

Remarks. Since $\Sigma$ is compact, the lemma implies that the self-intersection set $K$ itself is contained in a finite number of points and a finite number of $C^{1}$ curves of finite length. If $M$ has dimension three, Lemma 4.3 is a direct consequence of Lemma 2 in [25] or Lemma 1.4 in [16], and in fact the argument we present for Lemma 4.3 is just a modification of the argument given in [25].

To begin the proof, we first note that if $p \in K$, there are only finitely many points in $f^{-1}(f(p))$, or in other words only finitely many sheets of $f(\Sigma)$ which pass through $f(p)$. Indeed, if $f$ is minimal, the intersection of each sheet with a small geodesic ball of fixed radius around $f(p)$ has area bounded below by a fixed $\epsilon>0$ by (for example) Lemma 1, page 445 of [24], implying there can be only finitely many such sheets.

There is a simple idea which can be used to construct such an estimate, particularly if $f$ is an immersion. The key point is that (as mentioned in \$2) it follows directly from the Gauss equation for $f$ that the curvature of $f(\Sigma)$ in the induced metric is $\leq K_{0}$, where $K_{0}$ is the maximum value of the sectional curvatures of $M$. Moreover, a geodesic ball $B$ of radius $\delta>0$ about $f(p)$ in $M$ contains a geodesic ball of radius $\delta$ about $f(p)$ in $f(\Sigma)$. By using Jacobi fields along geodesics to compare with a space form of constant curvature $K_{0}$, one obtains a lower bound on the area of one sheet of $f(\Sigma) \cap B$ passing through $f(p)$. The case where $f$ is not an immersion is only a little more complicated; in this case, it is still possible to define geodesics emanating from $p$ (even though the induced metric is degenerate at $p$ ) and carry out the Jacobi field estimates. Thus a bound on the energy implies that there are only finitely many sheets passing through $f(p)$.

By an easy induction, we see that it suffices to consider the self-intersection set of two harmonic maps $f_{1}: D_{1} \rightarrow M$ and $f_{2}: D_{2} \rightarrow M$ from disks centered at $p_{1}$ and $p_{2}$ respectively such that $f_{1}\left(p_{1}\right)=f_{2}\left(p_{2}\right)=q$, as well as the self-intersection of a single such map near a branch point.

We start with the first case under the assumption that neither $p_{1}$ nor $p_{2}$ is a branch point. If $K$ is the intersection set of $f_{1}$ and $f_{2}$, we need to show that any point in $K$ lies in one of the two classes in the conclusion of the lemma. The tangent 
planes $\Pi_{1}$ and $\Pi_{2}$ at $q$ to $f_{1}\left(D_{1}\right)$ and $f_{2}\left(D_{2}\right)$, respectively, can intersect in a point or a line, or the two planes may coincide. If the planes intersect in a point, then $p_{1}$ and $p_{2}$ belong to the first class in the conclusion of the lemma.

If $\Pi_{1}$ and $\Pi_{2}$ intersect in a line, the planes make an angle $\theta$ with each other, where $0<\theta<\pi$. In this case there is a plane $\Pi$ which makes an acute angle with both $\Pi_{1}$ and $\Pi_{2}$. On the other hand, if $\Pi_{1}=\Pi_{2}$, we simply set $\Pi=\Pi_{1}=\Pi_{2}$. In either case, each of the harmonic surfaces can be regarded as a graph over $\Pi$ near $q$, in terms of normal coordinates on $M$ centered at $q$.

In more detail, we choose normal coordinates $\left(u_{1}, \ldots, u_{n}\right)$ on an open neighborhood of $q$ in $M$ with $u_{i}(q)=0$, so that the plane spanned by the coordinate vectors $\left(\partial / \partial u_{1}\right)$ and $\left(\partial / \partial u_{2}\right)$ at $q$ is $\Pi$. Let $B_{\epsilon}$ be a ball of radius $\epsilon$ about 0 in the $\left(u_{1}, u_{2}\right)$-plane. We can consider the two surfaces as graphs of functions

$$
h_{i}: B_{\epsilon} \rightarrow \mathbb{R}^{n-2}, \quad i=1,2,
$$

which satisfy an explicit nonlinear partial differential equation. Our convention is to denote the Riemannian metric on $M$ and the metric on $B_{\epsilon}$ induced by the immersion $\left(u_{1}, u_{2}\right) \mapsto\left(u_{1}, u_{2}, h_{i}\left(u_{1}, u_{2}\right)\right)$ by

$$
\sum_{i, j=1}^{n} g_{i j} d u_{i} d u_{j} \quad \text { and } \quad \sum_{a, b=1}^{2} \eta_{a b} d u_{a} d u_{a}
$$

respectively. With this convention, it follows from a relatively straightforward calculation (presented in the Appendix to [27]) that each $h_{i}$ is an $\mathbb{R}^{n-2}$-valued solution to an elliptic system in "nonparametric form",

$$
\sum_{a, b=1}^{2} \eta^{a b}\left(u_{1}, u_{2}, h, D h\right) \frac{\partial^{2} h}{\partial u_{a} \partial u_{b}}+\phi\left(u_{1}, u_{2}, h, D h\right)=0,
$$

where $\left(\eta^{a b}\right)$ is the matrix inverse of $\left(\eta_{a b}\right)$, the operator on the left once again having scalar symbol.

It is readily checked that the difference $h=h_{2}-h_{1}$ satisfies an equation of the same form,

$$
\sum_{a, b=1}^{2} \eta^{a b}\left(u_{1}, u_{2}, h, D h\right) \frac{\partial^{2} h}{\partial u_{a} \partial u_{b}}+\phi\left(u_{1}, u_{2}, h, D h\right)=0,
$$

in which the coefficients depend upon $h_{1}$, which is now regarded as a known function of $u_{1}$ and $u_{2}$, with $h$ or equivalently $h_{2}$ being regarded as unknown. Thus by Corollary 1 of Theorem 1.1 in [27] (a corollary of a theorem of Hartman and Wintner), we see that either $h \equiv 0$ or

$$
\begin{aligned}
& \left|h\left(u_{1}, u_{2}\right)\right|=O\left(\left|\left(u_{1}, u_{2}\right)\right|^{m}\right) \quad \text { for some positive integer } m, \text { and } \\
& \quad \Rightarrow \quad h\left(u_{1}, u_{2}\right)=p\left(u_{1}, u_{2}\right)+o_{1}\left(\left|\left(u_{1}, u_{2}\right)\right|^{m}\right),
\end{aligned}
$$

where $p\left(u_{1}, u_{2}\right)$ is a nonzero homogeneous vector-valued harmonic polynomial of degree $m$. We recall that stating that $h-p$ is $o_{1}\left(|z|^{m}\right)$ means that $h-p$ is $o\left(|z|^{m}\right)$ and $D(h-p)$ is $o\left(|z|^{m-1}\right)$. It cannot be the case that $h \equiv 0$ by Lemma 4.1, since $f$ is assumed to be prime.

Since the domain is only two-dimensional, such harmonic polynomials are easily described; indeed,

$$
p\left(u_{1}, u_{2}\right)=\operatorname{Re}\left(a\left(u_{1}+i u_{2}\right)^{m}\right), \quad \text { where } a \text { is a nonzero element of } \mathbb{C}^{n-2} .
$$


If $m=1$, the two surfaces intersect in a smooth curve near $q$. If $m>1$, this vector-valued harmonic polynomial vanishes at a single point or on a $C^{1}$ collection of curves. In the former case, the two surfaces intersect in an isolated point and we are done. In the latter case, we can choose a one-dimensional linear subspace $V$ of $\mathbb{R}^{n-2}$ such that if $\pi_{V}$ denotes the orthogonal projection into this subspace, $\pi_{V} \circ p$ is not identically zero. The zero set of the $\mathbb{R}^{n-2}$-valued function $h$ is contained in the zero set of the scalar function $\pi_{V} \circ h$, so it suffices to show that the zero set of $\pi_{V} \circ h$ is a finite collection of $C^{1}$ curves passing through the origin.

But now, following 25, we can apply Lemma 4.2 to conclude that there is a $C^{1}$ local diffeomorphism $\Phi$ near the origin in $\mathbb{R}^{2}$ such that $\pi_{V} \circ h=\pi_{V} \circ p \circ \Phi$. Thus $\pi_{V} \circ h$ vanishes on the intersection of a finite number of $C^{1}$ curves in terms of the parameter $\xi$, and by the canonical form described at the beginning of the section, also in terms of the conformal parameter $z$. This establishes the desired conclusion in this case.

To finish the proof in the case that branch points are present, recall that we need to consider:

(1) the self-intersection set of a single small minimal disk $f: D \rightarrow M$ in which $D$ is centered at a branch point $p$, and

(2) the intersection of two minimal disks $f_{1}: D_{1} \rightarrow M$ and $f_{2}: D_{2} \rightarrow M$ centered at $p_{1}$ and $p_{2}$, with $f\left(p_{1}\right)=f\left(p_{2}\right)=q$, in which one or both of the points $p_{1}$ and $p_{2}$ are branch points.

If we were to make the assumption that $f$ is not only minimal, but also locally area minimizing, we could apply Theorem 4.2 of [27] to show that the selfintersection set of the restriction of $f$ to a neighborhood of the branch point $p$ is empty when the neighborhood is sufficiently small, completing the first of these steps.

In general, the self-intersections near a branch point $p$ on a single minimal disk $f: D \rightarrow M$ can be treated by the canonical form (31). We note that for particular points $\xi_{1}$ and $\xi_{2}$ of $D, f\left(\xi_{1}\right)$ can equal $f\left(\xi_{2}\right)$ only if $\xi_{2}=\mu \xi_{1}$, where $\mu$ is a $(\nu+1)$ fold root of unity, and $\tilde{f}\left(\mu \xi_{1}\right)=\tilde{f}\left(\xi_{2}\right)$, where $\tilde{f}$ is defined by (32). According to Lemma 4.1 , since $f$ is prime, it is primitive, and hence $\tilde{f}(\mu \xi)-\tilde{f}(\xi)$ is not identically zero for any nontrivial $(\nu+1)$-root of unity. Thus we can apply (33) to conclude that

$$
\pi_{V} \circ \tilde{f}(\mu \xi)-\pi_{V} \circ \tilde{f}(\xi)=\pi_{V}\left(a \xi^{\rho}+\bar{a} \bar{\xi}^{\rho}\right)+o_{1}\left(|\xi|^{\rho}\right),
$$

where $\pi_{V}$ is the projection into a three-dimensional subspace of $\mathbb{R}^{n}$ containing the tangent plane at $p$ such that $\pi_{V}(a+\bar{a}) \neq 0$. Applying Lemma 4.2 once again shows that $\pi_{V} \circ \tilde{f}(\mu \xi)-\pi_{V} \tilde{f}(\xi)$ vanishes on a finite number of $C^{1}$ curves (in terms of the nonconformal parameter $\xi$ ) passing through $p$. Once again, since the transformation from the conformal parameter $z$ to $\xi$ is $C^{2, \alpha}$, the self-intersection set of $f \mid D$ lies within a finite collection of $C^{1}$ curves of finite length, finishing the argument in this case.

We next consider the case in which two minimal disks $f_{1}: D_{1} \rightarrow M$ and $f_{2}$ : $D_{2} \rightarrow M$ intersect at the centers $p_{1}$ and $p_{2}$ of the disks. If the tangent planes $\Pi_{1}$ and $\Pi_{2}$ to $f_{1}\left(D_{1}\right)$ and $f_{2}\left(D_{2}\right)$ at $q=f\left(p_{1}\right)=f\left(p_{2}\right)$ intersect at a point, then the two minimal disks also intersect at a point, and $p_{1}$ and $p_{2}$ are isolated points of self-intersection.

Suppose next that the two planes $\Pi_{1}$ and $\Pi_{2}$ coincide and $p_{1}$ is a branch point of order $\nu$, while $p_{2}$ is not a branch point. In this case, we replace $f_{2}: D_{2} \rightarrow M$ by 
a new disk $f_{2}^{\prime}: D_{2}^{\prime} \rightarrow M$ centered at $p_{2}^{\prime}$ such that $f_{2}^{\prime}(z)=f_{2}\left(z^{\nu+1}\right)$. Thus $f_{2}^{\prime}$ is a branched cover of $f_{2}$, and $f_{2}^{\prime}$ has a false branch point at its center $p_{2}^{\prime}$ with exactly the same order as the branch point at $p_{1}$. Both $f_{1}$ and $f_{2}^{\prime}$ have nonconformal parametrizations

$$
\xi \mapsto\left(\xi^{\nu+1}, \tilde{f}_{1}(\xi)\right), \quad \xi \mapsto\left(\xi^{\nu+1}, \tilde{f}_{2}^{\prime}(\xi)\right),
$$

in which $\xi$ is related to conformal parameters $z_{1}$ on $D_{1}$ and $z_{2}^{\prime}$ on $D_{2}^{\prime}$ by $C^{2, \alpha}$ diffeomorphisms. It cannot be the case that $\tilde{f}_{1}-\tilde{f}_{2}^{\prime}$ is identically zero, because this would contradict unique continuation, since $f$ is prime. Therefore it follows from Remark 1.6 of [27] that if $\mu$ is any nontrivial $(\nu+1)$-fold root of unity, then

$$
\tilde{f}_{1}(\mu \xi)-\tilde{f}_{2}^{\prime}(\xi)=a \xi^{\rho}+\bar{a} \bar{\xi}^{\rho}+o_{1}\left(|\xi|^{\rho}\right),
$$

where $a$ is a nonzero element of $\mathbb{C}^{n-2}$ and $\rho>\nu+1$. An application of Lemma 4.2 again shows that $f_{1}$ and $f_{2}^{\prime}$ intersect in a finite number of $C^{1}$ curves of finite length intersecting at $p_{1}$ or $p_{2}^{\prime}$, in terms of the parameter $\xi$. This holds also in terms of conformal parameters $z_{1}$ for $f_{1}$ and $z_{2}^{\prime}$ for $f_{2}^{\prime}$, just as before. The images of these curves in the disk $D_{2}$ are $C^{1}$ curves intersecting at $p_{2}$.

If $\Pi_{1}$ and $\Pi_{2}$ coincide and both $p_{1}$ and $p_{2}$ are branch points of branching order $\nu_{1}$ and $\nu_{2}$, respectively, we let $\nu+1$ be the lowest common multiple of $\nu_{1}+1$ and $\nu_{2}+1$. We choose positive integers $\sigma_{1}$ and $\sigma_{2}$ so that $\nu+1=\sigma_{1}\left(\nu_{1}+1\right)$ and $\nu+1=\sigma_{2}\left(\nu_{2}+1\right)$. For $i=1,2$, we then replace $f_{i}: D_{i} \rightarrow M$ by a new disk $f_{i}^{\prime}: D_{i}^{\prime} \rightarrow M$ such that $f_{i}^{\prime}(z)=f_{i}\left(z^{\sigma_{i}}\right)$. The two disks $f_{1}^{\prime}$ and $f_{2}^{\prime}$ are no longer necessarily primitive, but we can still apply Remark 1.6 of [27 to show that if $\mu$ is any $(\nu+1)$-root of unity, either

$$
\tilde{f}_{1}^{\prime}(\mu \xi)-\tilde{f}_{2}^{\prime}(\xi) \equiv 0 \quad \text { or } \quad \tilde{f}_{1}^{\prime}(\mu \xi)-\tilde{f}_{2}^{\prime}(\xi)=a \xi^{\rho}+\bar{a} \bar{\xi}^{\rho}+o_{1}\left(|\xi|^{\rho}\right),
$$

where $a$ is a nonzero element of $\mathbb{C}^{n-2}$ and $\rho>\nu+1$. Once again the first case would contradict unique continuation, since $f$ is prime, so we can apply Lemma 4.2 yet again to conclude that $f_{1}^{\prime}$ and $f_{2}^{\prime}$ intersect in a finite collection of $C^{1}$ curves of finite length, and once again we argue that $f_{1}$ and $f_{2}$ themselves intersect in a finite number of $C^{1}$ curves of finite length.

Finally, we need to consider the case in which the two planes $\Pi_{1}$ and $\Pi_{2}$ intersect in a line. As before, if $p_{1}$ is a branch point of order $\nu$, while $p_{2}$ is not a branch point, we replace $f_{2}$ by a branched cover $f_{2}^{\prime}$ so that $f_{1}^{\prime}$ and $f_{2}^{\prime}$ both have branch points of order $\nu$. If $p_{1}$ and $p_{2}$ are branch points of order $\nu_{1}$ and $\nu_{2}$ we let $\nu+1$ be the lowest common multiple of $\nu_{1}+1$ and $\nu_{2}+1$, and replace both $f_{1}$ and $f_{2}$ by branched covers $f_{1}^{\prime}$ and $f_{2}^{\prime}$ so that $f_{1}^{\prime}$ and $f_{2}^{\prime}$ both have branching order $\nu$.

Each of the maps $f_{1}^{\prime}$ and $f_{2}^{\prime}$ has a canonical form (30), but since the tangent planes make an angle with each other, the normal coordinates in $M$ that are used to realize these forms are also at an angle. Thus we can choose two normal coordinate systems

$$
\left(u_{1}, u_{2}, u_{3}, u_{4}, \ldots, u_{n}\right) \quad \text { and } \quad\left(u_{1}, v_{2}, v_{3}, u_{4}, \ldots, u_{n}\right)
$$

related to each other by a rotation,

$$
u_{2}=a v_{2}-b v_{3}, \quad u_{3}=b v_{2}+a v_{3}, \quad a^{2}+b^{2}=1,
$$


where $b \neq 0$, so that by (31),

$$
\begin{aligned}
& u_{1}\left(\xi_{1}\right)+i u_{2}\left(\xi_{i}\right)=\xi_{1}^{\nu+1}, \quad u_{r}\left(\xi_{1}\right)=o_{1}\left(\left|\xi_{1}\right|^{\nu+1}\right) \quad \text { for } \quad 3 \leq r \leq n, \\
& u_{1}\left(\xi_{2}\right)+i v_{2}\left(\xi_{2}\right)=\xi_{2}^{\nu+1}, \quad v_{3}\left(\xi_{2}\right)=o_{1}\left(\left|\xi_{2}\right|^{\nu+1}\right), \quad u_{s}\left(\xi_{2}\right)=o_{1}\left(\left|\xi_{2}\right|^{\nu+1}\right) \\
& \text { for } 4 \leq s \leq n \text {, }
\end{aligned}
$$

where $\xi_{1}$ and $\xi_{2}$ are nonconformal parameters on $D_{1}$ and $D_{2}$ respectively. Note that $f_{1}^{\prime}$ and $f_{2}^{\prime}$ can intersect only where $\operatorname{Re}\left(\xi_{1}\right)=\operatorname{Re}\left(\xi_{2}\right)$. Since both $\xi_{1}$ and $\xi_{2}$ give Euclidean parametrizations of $\Pi_{1}$ and $\Pi_{2}$ respectively, we can arrange that $\xi_{1}$ and $\xi_{2}$ are related by a local diffeomorphism from a neighborhood of 0 in $D_{1}$ to a neighborhood of 0 in $D_{2}$ under which $\xi_{1} \mapsto \xi_{2}$ with $u_{1}\left(\xi_{1}\right)=u_{1}\left(\xi_{2}\right)$.

It follows from (34) that

$$
\begin{gathered}
u_{3}\left(\xi_{2}\right)=b \operatorname{Im}\left(\xi_{1}^{\nu+1}\right)+o_{1}\left(\left|\xi_{1}\right|^{\nu+1}\right)=b \operatorname{Im}\left(\xi_{2}^{\nu+1}\right)+o_{1}\left(\left|\xi_{2}\right|^{\nu+1}\right), \\
v_{3}\left(\xi_{1}\right)=-b \operatorname{Im}\left(\xi_{2}^{\nu+1}\right)+o_{1}\left(\left|\xi_{2}\right|^{\nu+1}\right)=-b \operatorname{Im}\left(\xi_{1}^{\nu+1}\right)+o_{1}\left(\left|\xi_{1}\right|^{\nu+1}\right) .
\end{gathered}
$$

According to Lemma 4.2, we can find local $C^{1}$ diffeomorphisms $\Phi_{1}$ and $\Phi_{2}$ of $D_{1}$ and $D_{2}$ so that if $\Phi_{i}\left(\eta_{i}\right)=\xi_{i}$, then

$$
v_{3} \circ \Phi_{1}\left(\eta_{1}\right)=-b \operatorname{Im}\left(\eta_{1}^{\nu+1}\right), \quad u_{3} \circ \Phi_{2}\left(\eta_{2}\right)=b \operatorname{Im}\left(\eta_{2}^{\nu+1}\right) .
$$

The set of points in $D_{1}$ taken by $f_{1}^{\prime}: D_{1} \rightarrow M$ to points of $f_{2}^{\prime}\left(D_{2}\right)$ are points such that $\operatorname{Im}\left(\eta_{1}^{\nu+1}\right)=0$, while the points of $D_{2}$ taken by $f_{2}^{\prime}: D_{2} \rightarrow M$ to points of $f_{1}^{\prime}\left(D_{1}\right)$ are points such that $\operatorname{Im}\left(\eta_{2}^{\nu+1}\right)=0$. Thus we find that the points of $K$ contributed in a neighborhood of a self-intersection of two disks whose tangent planes intersect in a line lie on finitely many $C^{1}$ curves of finite length.

Thus we have covered all cases and Lemma 4.3 is proven.

\section{SECOND VARIATION OF ENERGY}

So far we have discussed critical points for the function $E_{\omega}$ in which the conformal structure is fixed. For the theory of parametrized minimal surfaces in a given Riemannian manifold $M$, however, the function of primary importance is the twovariable energy

$$
E: \operatorname{Map}(\Sigma, M) \times \mathcal{T} \longrightarrow \mathbb{R}
$$

whose critical points are minimal surfaces, which may contain branch points. The second variation formula is slightly different in this case, since we must consider variations also in the $\mathcal{T}$-direction, where $\mathcal{T}$ is the Teichmüller space for compact Riemann surfaces of genus $g$.

From the formal point of view, the first derivative of the energy $E$ is given by the first variation formula

$$
d E(f, \omega)((X, \dot{\omega}))=\int_{\Sigma}\langle F(f, \omega, g),(X, \dot{\omega})\rangle d A
$$

where $F(\cdot, \cdot, g)=0$ is the Euler-Lagrange equation for the variational problem, $\langle\cdot, \cdot\rangle$ is an appropriate inner product on the tangent space to $\operatorname{Map}(\Sigma, M) \times \mathcal{T}$ and $(X, \dot{\omega})$ is an element of this tangent space. If $(f, \omega)$ is a critical point for $E$, we can 
differentiate once again, obtaining the second variation formula,

$$
\begin{aligned}
d^{2} E(f)\left(\left(X, \dot{\omega}_{1}\right),\left(Y, \dot{\omega}_{2}\right)\right)=\int_{\Sigma}\left\langle D_{1} F(f, \omega, g)\left(X, \dot{\omega}_{1}\right),\left(Y, \dot{\omega}_{2}\right)\right\rangle d A \\
=\int_{\Sigma}\left\langle L_{(f, \omega, g)}\left(X, \dot{\omega}_{1}\right),\left(Y, \dot{\omega}_{2}\right)\right\rangle d A
\end{aligned}
$$

where $D_{1} F$ denotes the derivative with respect to the variable

$$
(f, \omega) \in \operatorname{Map}(\Sigma, M) \times \mathcal{T} .
$$

If we let $L_{k}^{2}(\Sigma, M)$ denote the completion of $\operatorname{Map}(\Sigma, M)$ with respect to the $L_{k}^{2}$ norm, the Jacobi operator $L_{(f, \omega, g)}$ can be regarded as a Fredholm map,

$$
L_{(f, \omega, g)}: T_{(f, \omega)}\left(L_{k}^{2}(\Sigma, M) \times \mathcal{T}\right) \rightarrow T_{(f, \omega)}\left(L_{k-2}^{2}(\Sigma, M) \times \mathcal{T}\right) .
$$

Elements $(X, \dot{\omega})$ which satisfy the Jacobi equation $L_{(f, \omega, g)}(X, \dot{\omega})=0$ are the Jacobi fields at $(f, \omega)$ for $E$. Note that the Jacobi equation is the linearization of the Euler-Lagrange equation, since $L_{(f, \omega, g)}=D_{1} F(f, \omega, g)$.

As preparation for an analysis of first and second variation, we recall the definition of Teichmüller space when $g \geq 1$, as presented in Chapter 2 of [34], earlier presentations along similar lines being given in 12 and 14. We begin with the space $\operatorname{Met}(\Sigma)$ of smooth Riemannian metrics on the compact oriented surface $\Sigma$ of genus $g$, and consider the subspace $\operatorname{Met}_{0}(\Sigma)$ of metrics that have constant curvature and total area one. Since there is a unique such metric in each conformal equivalence class, we can regard $\operatorname{Met}_{0}(\Sigma)$ as being isomorphic to the space $\operatorname{Conf}(\Sigma)$ of conformal structures on $\Sigma$. There is an obvious action of the group $\operatorname{Diff}^{+}(\Sigma)$ of orientation-preserving diffeomorphisms on $\operatorname{Met}(\Sigma)$, given by

$$
(\eta, \phi) \in \operatorname{Met}(\Sigma) \times \operatorname{Diff}^{+}(\Sigma) \mapsto \phi^{*} \eta \in \operatorname{Met}(\Sigma)
$$

which restricts to an action of the normal subgroup

$$
\operatorname{Diff}_{0}(\Sigma)=\{\text { diffeomorphisms of } \Sigma \text { isotopic to the identity }\} \text {. }
$$

The Teichmüller space $\mathcal{T}$ of marked conformal structures for compact surfaces of genus $g$ is simply the quotient of the subspace $\operatorname{Met}_{0}(\Sigma)$ by this action:

$$
\mathcal{T}=\operatorname{Met}_{0}(\Sigma) / \operatorname{Diff}_{0}(\Sigma)=\operatorname{Conf}(\Sigma) / \operatorname{Diff}_{0}(\Sigma) .
$$

The quotient by the full group $\operatorname{Diff}^{+}(\Sigma)$ gives the moduli space $\mathcal{R}$ of conformal structures on $\Sigma$ (without marking), while the quotient $\Gamma=\operatorname{Diff}^{+}(\Sigma) / \operatorname{Diff}_{0}(\Sigma)$ is the mapping class group referred to in the Introduction.

We can now give a more precise description of the two-variable energy function $E$. First, we write a typical element $\eta \in \operatorname{Met}_{0}(\Sigma)$ in terms of fixed coordinates $\left(x_{1}, x_{2}\right)$ on $\Sigma$ as

$$
\eta=\sum_{a, b=1}^{2} \eta_{a b} d x_{a} d x_{b}, \quad \text { and let } \quad\left(\eta^{a b}\right)=\left(\eta_{a b}\right)^{-1} .
$$

We can then define $E: \operatorname{Map}(\Sigma, M) \times \operatorname{Met}(\Sigma) \rightarrow \mathbb{R}$ by

$$
E(f, \eta)=\frac{1}{2} \int_{\Sigma} \sum_{a, b} \eta^{a b} \sqrt{\operatorname{det}\left(\eta_{a b}\right)}\left\langle\frac{\partial f}{\partial x_{a}}, \frac{\partial f}{\partial x_{b}}\right\rangle d x_{1} d x_{2} .
$$

The group $\operatorname{Diff}^{+}(\Sigma)$ acts on $\operatorname{Map}(\Sigma, M)$ by

$$
(f, \phi) \in \operatorname{Map}(\Sigma, M) \times \operatorname{Diff}^{+}(\Sigma) \mapsto f \circ \phi,
$$


and the energy $E$ is invariant under the corresponding product action of Diff ${ }^{+}(\Sigma)$ on $\operatorname{Map}(\Sigma, M) \times \operatorname{Met}_{0}(\Sigma)$. The function $E$ thus descends to a map on the quotient

$$
\mathcal{M}(\Sigma, M)=\frac{\operatorname{Map}(\Sigma, M) \times \operatorname{Met}_{0}(\Sigma)}{\operatorname{Diff}^{+}(\Sigma)} .
$$

As mentioned in the Introduction, this space is the most natural domain for developing a Morse theory for the energy $E$.

However, it is much simpler at present to regard the energy as a function on the space

$$
\widetilde{\mathcal{M}}(\Sigma, M)=\frac{\operatorname{Map}(\Sigma, M) \times \operatorname{Met}_{0}(\Sigma)}{\operatorname{Diff}_{0}(\Sigma)} .
$$

The advantage is that the projection on the second factor

$$
\pi: \operatorname{Map}(\Sigma, M) \times \operatorname{Met}_{0}(\Sigma) \rightarrow \operatorname{Met}_{0}(\Sigma)
$$

descends to a fiber bundle projection from $\widetilde{\mathcal{M}}(\Sigma, M)$ to $\mathcal{T}$, the latter space being diffeomorphic to a ball by a well-known theorem from Teichmüller theory. An explicit diffeomorphism is given in Chapter 2, $\S 3$ of [34, and using it, we can construct a trivialization of the bundle; see also [12. Thus we can also regard the energy $E$ as a function on the product $\operatorname{space} \operatorname{Map}(\Sigma, M) \times \mathcal{T}$, in agreement with (35), and if $[f, \eta]$ denotes the equivalence class of $(f, \eta) \in \operatorname{Map}(\Sigma, M) \times \operatorname{Met}_{0}(\Sigma)$, we can identify

$$
[f, \eta] \in \widetilde{\mathcal{M}}(\Sigma, M) \quad \text { with } \quad(f, \omega) \in \operatorname{Map}(\Sigma, M) \times \mathcal{T}
$$

$\omega$ being the conformal class of the metric $\eta$.

To calculate the first derivative of $E$ on $\widetilde{\mathcal{M}}(\Sigma, M)$, we can first calculate the derivative of

$$
E: \operatorname{Map}(\Sigma, M) \times \operatorname{Met}(\Sigma) \rightarrow \mathbb{R}
$$

then restrict to the tangent space to $\operatorname{Map}(\Sigma, M) \times \operatorname{Met}_{0}(\Sigma)$, and finally restrict to the tangent space to a slice for the action of $\operatorname{Diff}_{0}(\Sigma)$ which is transversal to the orbits. (Throughout the calculation we assume, of course, that the genus of $\Sigma$ is at least one.) This slice is similar to the slice for the action on $\operatorname{Met}_{0}(\Sigma)$ which is described in Chapter 2, $\S 2$ of [34]. In our case, the slice which passes through a representative

$$
(f, \eta) \in \operatorname{Map}(\Sigma, M) \times \operatorname{Met}_{0}(\Sigma) \quad \text { for } \quad[f, \eta] \in \widetilde{\mathcal{M}}(\Sigma, M)
$$

allows us to make the identification

$$
T_{[f, \eta]} \widetilde{\mathcal{M}}(\Sigma, M) \cong T_{f} \operatorname{Map}(\Sigma, M) \oplus T_{\omega} \mathcal{T} .
$$

In the metric direction, the slice is chosen to be tangent to the one-parameter families of metrics of the form $\eta_{i j}(t)=\eta_{i j}(0)+t \dot{\eta}_{i j}$, with $\eta_{i j}(0)=\lambda^{2} \delta_{i j}$ for some conformal factor $\lambda^{2}$, the $\left(\dot{\eta}_{i j}\right)$ being required to be $L^{2}$-perpendicular to the orbits of $\operatorname{Diff}_{0}(\Sigma)$ at $(f, \eta)$. When the genus is at least two, the argument in [34 shows that for this choice of slice, $\dot{\eta}$ satisfies the zero-trace condition $\dot{\eta}_{11}+\dot{\eta}_{22}=0$, and the condition that

$$
h d z^{2}=\left(\dot{\eta}_{11}-i \dot{\eta}_{12}\right) d z^{2}
$$

be a holomorphic quadratic differential. If the genus is one, our normalization of the area forces the trace-free condition, and an argument similar to that in [34] 
shows that $h d z^{2}$ is once again holomorphic. The calculation will yield a formula for

$$
d E([f, \eta]): T_{[f, \eta]} \widetilde{\mathcal{M}}(\Sigma, M) \longrightarrow \mathbb{R}
$$

which is called the first variation of $E$ at $[f, \eta]$. At a critical point, this differential must vanish when restricted to $T_{f} \operatorname{Map}(\Sigma, M) \oplus\{0\}$, and hence $f$ must be a harmonic map.

We want to calculate the derivative in the other direction, without necessarily making the assumption that $f$ is harmonic. We will use the symbol $\eta$ for both the metric on $\Sigma$ itself and for $\operatorname{det}\left(\eta_{a b}\right)$, the context hopefully making clear which is meant. We differentiate (38), taking a perturbation given by the formula

$$
\eta_{a b}(t)=\eta_{a b}+t \dot{\eta}_{a b}
$$

where the variation $\dot{\eta}_{a b}$ in the metric is trace-free $\left(\dot{\eta}_{11}+\dot{\eta}_{22}=0\right)$ and the initial metric is given by the formula

$$
\eta_{a b}=\delta_{a b} \lambda^{2}, \quad \sqrt{\eta}=\sqrt{\operatorname{det}\left(\eta_{a b}\right)}=\lambda^{2},
$$

for some conformal factor $\lambda^{2}$. Then

$$
\frac{d}{d t} \eta_{a b}(t)=\dot{\eta}_{a b},\left.\quad \frac{d}{d t} \eta(t)\right|_{t=0}=\lambda^{2}\left(\dot{\eta}_{11}+\dot{\eta}_{22}\right)=0 .
$$

From these formulae, we can easily conclude that

$$
\left.\frac{d}{d t}\left(\begin{array}{cc}
\sqrt{\eta} \eta^{11} & \sqrt{\eta} \eta^{12} \\
\sqrt{\eta} \eta^{21} & \sqrt{\eta} \eta^{22}
\end{array}\right)\right|_{t=0}=\lambda^{-2}\left(\begin{array}{cc}
\dot{\eta}_{22} & -\dot{\eta}_{12} \\
-\dot{\eta}_{21} & \dot{\eta}_{11}
\end{array}\right) .
$$

Thus we find that

$$
\begin{aligned}
&\left.\frac{d}{d t} E\left(f, \eta_{a b}(t)\right)\right|_{t=0}=\left.\frac{1}{2} \int_{\Sigma} \sum_{a, b} \frac{d}{d t} \sqrt{\eta} \eta^{a b}\right|_{t=0}\left\langle\frac{\partial f}{\partial x_{a}}, \frac{\partial f}{\partial x_{b}}\right\rangle d x_{1} d x_{2} \\
&=-\frac{1}{2} \int_{\Sigma}\left[\frac{\dot{\eta}_{11}}{\lambda^{2}}\left(\left\langle\frac{\partial f}{\partial x_{1}}, \frac{\partial f}{\partial x_{1}}\right\rangle-\left\langle\frac{\partial f}{\partial x_{2}}, \frac{\partial f}{\partial x_{2}}\right\rangle\right)+\frac{2 \dot{\eta}_{12}}{\lambda^{2}}\left\langle\frac{\partial f}{\partial x_{1}}, \frac{\partial f}{\partial x_{2}}\right\rangle\right] d x_{1} d x_{2} \\
&=-2 \int_{\Sigma} \frac{1}{\lambda^{2}} \operatorname{Re}\left[\left(\dot{\eta}_{11}+i \dot{\eta}_{12}\right)\left\langle\frac{\partial f}{\partial z}, \frac{\partial f}{\partial z}\right\rangle\right] d x_{1} d x_{2} .
\end{aligned}
$$

We need to understand (42) in terms of the tangent space $T_{\omega} \mathcal{T}$ to Teichmüller space, which can be regarded as the space of trace-free metric deformations $\left(\dot{\eta}_{a b}\right)$ such that $\left(\dot{\eta}_{11}-i \dot{\eta}_{12}\right) d z^{2}$ is a holomorphic quadratic differential. It is customary to regard the space of holomorphic quadratic differentials as the the cotangent space to $\mathcal{T}$. Given the canonical metric, a holomorphic quadratic differential $h d z^{2}$ on $\Sigma$ determines the corresponding extremal Beltrami differential by raising the index

$$
\left(h d z^{2}\right)^{\sharp}=\mu \frac{d \bar{z}}{d z}=\frac{\bar{h} d \bar{z}^{2}}{\lambda^{2} d z d \bar{z}}=\frac{\bar{h}}{\lambda^{2}} \frac{d \bar{z}}{d z},
$$

while the extremal Beltrami differential $\mu(d \bar{z} / d z)$ determines a corresponding holomorphic quadratic differential

$$
\left(\mu \frac{d \bar{z}}{d z}\right)^{b}=\bar{\mu} \lambda^{2} d z^{2}
$$

It is customary to regard the tangent space $T \mathcal{T}$ to Teichmüller space as being the space of extremal Beltrami differentials. (In the classical Teichmüller theory [7, 
the extremal Beltrami differential in the tangent space corresponds to an extremal quasiconformal map.)

We define an $L^{2}$ Hermitian inner product $\langle\langle\cdot, \cdot\rangle\rangle$ on the space of all quadratic differentials by

$$
\left\langle\left\langle h d z^{2}, k d z^{2}\right\rangle\right\rangle=2 \int_{\Sigma} \frac{1}{\lambda^{4}} h \bar{k} d A,
$$

and when restricted to holomorphic quadratic differentials, this determines a Hermitian inner product on the holomorphic cotangent space to Teichmüller space, as well as a corresponding Hermitian inner product on the holomorphic tangent space, called the Weil-Petersson metric.

Using this Hermitian inner product, we can rewrite (42) as

$$
\left.\frac{d}{d t} E\left(f, \eta_{a b}(t)\right)\right|_{t=0}=-\operatorname{Re}\left\langle\left\langle\Omega_{f},\left(\dot{\eta}_{11}-i \dot{\eta}_{12}\right) d z^{2}\right\rangle\right\rangle
$$

where $\Omega_{f}$ is the Hopf differential, which can be defined of course even if $f$ is not harmonic. Thus the vanishing of the restriction of $d E([f, \eta])$ to $\{0\} \oplus T_{\omega} \mathcal{I}_{g}$ is equivalent to the condition

$$
P\left(\Omega_{f}\right)=0, \text { where } P=\text { projection to holomorphic quadratic differentials, }
$$

the projection being $L^{2}$-orthogonal. Conformal maps satisfy this condition even if $f$ is not harmonic.

At a critical point for $E$ itself, the Hopf differential $\Omega_{f}$, which is now holomorphic, must vanish. We thus recover the familiar fact that critical points for $E$ are indeed conformal harmonic maps, possibly with branch points. More generally, the above discussion is summarized by:

Proposition 5.1. The first variation for energy is given by the formula

$$
\begin{gathered}
d E(f, \omega)((X, \dot{\omega}))=\int_{\Sigma}\left[\left\langle\frac{\partial f}{\partial x}, \frac{D X}{\partial x}\right\rangle+\left\langle\frac{\partial f}{\partial y}, \frac{D X}{\partial y}\right\rangle\right] d x d y-\operatorname{Re}\left\langle\left\langle\Omega_{f},(\dot{\omega})^{b}\right\rangle\right\rangle \\
=-\int_{\Sigma}\left[\left\langle\frac{D}{\partial x}\left(\frac{\partial f}{\partial x}\right)+\frac{D}{\partial y}\left(\frac{\partial f}{\partial y}\right), X\right\rangle\right] d x d y-\operatorname{Re}\left\langle\left\langle\Omega_{f},(\dot{\omega})^{b}\right\rangle\right\rangle .
\end{gathered}
$$

Here $\dot{\omega}$ is an extremal Beltrami differential, regarded as an element of the holomorphic tangent space to Teichmüller space $\mathcal{T}$, which corresponds to the holomorphic quadratic differential $(\dot{\omega})^{b}$.

We now turn to the main goal of this section, and calculate the second variation at a critical point $[f, \eta]=(f, \omega)$ for $E$ using the trace-free metric variation (41). A direct calculation shows that

$$
\begin{gathered}
\frac{d}{d t} \eta_{a b}(t)=\dot{\eta}_{a b}, \quad \frac{d^{2}}{d t^{2}} \eta_{a b}=0 \\
\frac{d}{d t} \eta(t)=\dot{\eta}_{11} \eta_{22}(t)+\eta_{11}(t) \dot{\eta}_{22}-2 \eta_{12}(t) \dot{\eta}_{12}, \quad \frac{d^{2}}{d t^{2}} \eta(t)=2 \dot{\eta}_{11} \dot{\eta}_{22}-2 \dot{\eta}_{12} \dot{\eta}_{12} .
\end{gathered}
$$

Using the formulae

$$
\frac{d}{d t}\left(\eta^{-1 / 2}\right)=-\frac{1}{2} \eta^{-3 / 2} \frac{d \eta}{d t}, \quad \frac{d^{2}}{d t^{2}}\left(\eta^{-1 / 2}\right)=-\frac{1}{2} \eta^{-3 / 2} \frac{d^{2} \eta}{d t^{2}}+\frac{3}{4} \eta^{-5 / 2}\left(\frac{d \eta}{d t}\right)^{2}
$$


it is possible to differentiate

$$
\left(\begin{array}{cc}
\sqrt{\eta} \eta^{11} & \sqrt{\eta} \eta^{12} \\
\sqrt{\eta} \eta^{21} & \sqrt{\eta} \eta^{22}
\end{array}\right)=\eta^{-1 / 2}\left(\begin{array}{cc}
\eta_{22} & -\eta_{12} \\
-\eta_{21} & \eta_{11}
\end{array}\right)
$$

A calculation yields the results

$$
\frac{d}{d t}\left(\begin{array}{cc}
\sqrt{\eta} \eta^{11} & \sqrt{\eta} \eta^{12} \\
\sqrt{\eta} \eta^{21} & \sqrt{\eta} \eta^{22}
\end{array}\right)=\frac{d}{d t}\left(\eta^{-1 / 2}\right)\left(\begin{array}{cc}
\eta_{22} & -\eta_{12} \\
-\eta_{21} & \eta_{11}
\end{array}\right)+\eta^{-1 / 2}\left(\begin{array}{cc}
\dot{\eta}_{22} & -\dot{\eta}_{12} \\
-\dot{\eta}_{21} & \dot{\eta}_{11}
\end{array}\right)
$$

and

$$
\begin{aligned}
\frac{d^{2}}{d t^{2}}\left(\begin{array}{cc}
\sqrt{\eta} \eta^{11} & \sqrt{\eta} \eta^{12} \\
\sqrt{\eta} \eta^{21} & \sqrt{\eta} \eta^{22}
\end{array}\right) & =\frac{d^{2}}{d t^{2}}\left(\eta^{-1 / 2}\right)\left(\begin{array}{cc}
\eta_{22} & -\eta_{12} \\
-\eta_{21} & \eta_{11}
\end{array}\right)+2 \frac{d}{d t}\left(\eta^{-1 / 2}\right)\left(\begin{array}{cc}
\dot{\eta}_{22} & -\dot{\eta}_{12} \\
-\dot{\eta}_{21} & \dot{\eta}_{11}
\end{array}\right) .
\end{aligned}
$$

At $t=0$, we have

$$
\left(\begin{array}{ll}
\eta_{11} & \eta_{12} \\
\eta_{21} & \eta_{22}
\end{array}\right)=\left(\begin{array}{cc}
\lambda^{2} & 0 \\
0 & \lambda^{2}
\end{array}\right), \quad \eta=\lambda^{4}
$$

Moreover, since $\dot{\eta}_{11}+\dot{\eta}_{22}=0$, we readily verify that

$$
\left.\frac{d}{d t}(\eta)\right|_{t=0}=0,\left.\quad \frac{d^{2}}{d t^{2}}(\eta)\right|_{t=0}=-2\left(\dot{\eta}_{11}^{2}+\dot{\eta}_{12}^{2}\right)
$$

and our formulae for the derivatives of $\sqrt{\eta} \eta^{a b}$ simplify:

$$
\begin{gathered}
\left.\frac{d}{d t}\left(\begin{array}{cc}
\sqrt{\eta} \eta^{11} & \sqrt{\eta} \eta^{12} \\
\sqrt{\eta} \eta^{21} & \sqrt{\eta} \eta^{22}
\end{array}\right)\right|_{t=0}=\lambda^{-2}\left(\begin{array}{ll}
-\dot{\eta}_{11} & -\dot{\eta}_{12} \\
-\dot{\eta}_{21} & -\dot{\eta}_{22}
\end{array}\right) \\
\left.\frac{d^{2}}{d t^{2}}\left(\begin{array}{cc}
\sqrt{\eta} \eta^{11} & \sqrt{\eta} \eta^{12} \\
\sqrt{\eta} \eta^{21} & \sqrt{\eta} \eta^{22}
\end{array}\right)\right|_{t=0}=\lambda^{-4}\left(\dot{\eta}_{11}^{2}+\dot{\eta}_{12}^{2}\right)\left(\begin{array}{ll}
1 & 0 \\
0 & 1
\end{array}\right) .
\end{gathered}
$$

Finally, at a critical point for energy, we must have

$$
\left\langle\frac{\partial f}{\partial x_{a}}, \frac{\partial f}{\partial x_{b}}\right\rangle=\delta_{a b}\left\langle\frac{\partial f}{\partial x_{1}}, \frac{\partial f}{\partial x_{1}}\right\rangle=\delta_{a b} \sigma^{2}
$$

where the Riemannian metric induced by $f$ on $\Sigma$ is $\sigma^{2}\left(d x_{1}^{2}+d x_{2}^{2}\right)$.

With these preliminaries out of the way, we can now calculate the second derivative of energy, and find that it is given by a sum of three terms:

$$
\begin{aligned}
d^{2} E(f, \omega)((X, \dot{\eta}),(X, \dot{\eta})) & =d^{2} E_{\omega}(f)(X, X) \\
+2 \int_{\Sigma} \sum_{a, b} \frac{d}{d t} & \left.\left(\eta^{a b} \sqrt{\eta}\right)\right|_{t=0}\left\langle\frac{D X}{\partial x_{a}}, \frac{\partial f}{\partial x_{b}}\right\rangle d x_{1} d x_{2} \\
& +\left.\frac{1}{2} \int_{\Sigma} \sum_{a, b} \frac{d^{2}}{d t^{2}}\left(\eta^{a b} \sqrt{\eta}\right)\right|_{t=0}\left\langle\frac{\partial f}{\partial x_{a}}, \frac{\partial f}{\partial x_{b}}\right\rangle d x_{1} d x_{2},
\end{aligned}
$$

the first term being the usual second variation of $\omega$-energy, $\omega$ being the conformal equivalence class of the metric $\eta$. Here $X$ is a section of $f^{*} T M$ and $\dot{\eta}$ is a variation in the constant curvature metric tangent to our slice. We can think of these three terms as second-order partial derivatives in the three directions $\operatorname{Map}(\Sigma, M) \times \operatorname{Map}(\Sigma, M)$, $\operatorname{Map}(\Sigma, M) \times \mathcal{T}$ and $\mathcal{T} \times \mathcal{T}$. Using (48), (49) and (50), together with polarization, we obtain the following. 
Proposition 5.2. The second variation for energy is given by the formula

$$
\begin{aligned}
& d^{2} E(f, \omega)((X, \dot{\eta}),(Y, \dot{\zeta}))=d^{2} E_{\omega}(f)(X, Y) \\
& -\int_{\Sigma} \sum_{a, b}\left[\frac{\dot{\eta}_{a b}}{\lambda^{2}}\left\langle\frac{D Y}{\partial x_{a}}, \frac{\partial f}{\partial x_{b}}\right\rangle d x_{1} d x_{2}+\sum_{a, b} \frac{\dot{\zeta}_{a b}}{\lambda^{2}}\left\langle\frac{D X}{\partial x_{a}}, \frac{\partial f}{\partial x_{b}}\right\rangle\right] d x_{1} d x_{2} \\
& +\int_{\Sigma} \frac{1}{\lambda^{4}}\left(\dot{\eta}_{11} \dot{\zeta}_{11}+\dot{\eta}_{12} \dot{\zeta}_{12}\right) \sigma^{2} d x_{1} d x_{2},
\end{aligned}
$$

where $X$ and $Y$ are sections of $f^{*} T M$ and $\dot{\eta}$ and $\dot{\zeta}$ are variations in the metric tangent to the slice for the $\operatorname{Diff}_{0}(\Sigma)$ action on $\operatorname{Met}_{0}(\Sigma)$.

We could use the second variation formula to determine an expression for the corresponding Jacobi operator $L_{f, \omega, g}$ defined by (37). The inner product that we use in (37) is the direct sum of the pullback Riemannian metric on $f^{*} T M$ and the Weil-Petersson metric on Teichmüller space.

Of course, we can complexify the second variation formula (52) and consider the corresponding complex linear extension of the Jacobi operator, also denoted by $L_{f, \omega, g}$, and defined by

$$
\begin{aligned}
d^{2} E(f, \omega)\left(\left(Z_{1}, \dot{\omega}_{1}\right),\left(\bar{Z}_{2}, \bar{\omega}_{2}\right)\right)=\int_{\Sigma}\left\langle L_{f, \omega, g}\left(Z_{1}, \dot{\omega}_{1}\right),\left(\bar{Z}_{2}, \overline{\dot{\omega}_{2}}\right)\right\rangle d A \\
\quad=\int_{\Sigma}\left\langle\left\langle L_{f, \omega, g}\left(Z_{1}, \dot{\omega}_{1}\right),\left(Z_{2}, \dot{\omega}_{2}\right)\right\rangle\right\rangle d A
\end{aligned}
$$

Here $\langle\cdot, \cdot\rangle$ denotes the complex bilinear extension of the inner product used in (37), $\langle\langle\cdot, \cdot\rangle\rangle$ is the corresponding Hermitian inner product, and $\omega_{1}$ and $\omega_{2}$ are extremal Beltrami differentials. The extended Jacobi operator $L_{f, \omega, g}$ has two components

$$
L_{0}: \Gamma(\mathbf{E}) \oplus\left(T_{\omega} \mathcal{T} \otimes \mathbb{C}\right) \rightarrow \Gamma(\mathbf{E}), \quad L_{1}: \Gamma(\mathbf{E}) \oplus\left(T_{\omega} \mathcal{T} \otimes \mathbb{C}\right) \rightarrow T_{\omega} \mathcal{T} \otimes \mathbb{C},
$$

where $\mathbf{E}=f^{*} T M \otimes \mathbb{C}$.

Tangential Jacobi fields will play a key role in the proof of the Main Theorem. If $X$ is a section of $\left(f^{*} T M\right)^{\top}$, we claim that the first term in the second variation formula (51) can be written as

$$
d^{2} E_{\omega}(f)(X, X)=\int_{\Sigma}\left\langle\frac{D X}{\partial x_{1}}+J \frac{D X}{\partial x_{2}}, \frac{D X}{\partial x_{1}}+J \frac{D X}{\partial x_{2}}\right\rangle d x_{1} d x_{2},
$$

where $J$ is the endomorphism of $\left(f^{*} T M\right)^{\top}$ defined by

$$
J\left(\frac{\partial f}{\partial x}\right)=\frac{\partial f}{\partial y}, \quad J\left(\frac{\partial f}{\partial y}\right)=-\frac{\partial f}{\partial x} .
$$

Indeed, under the isomorphism $\tau:\left(f^{*} T M\right)^{\top} \rightarrow \mathbf{L}$ defined by (9), $J$ gets taken to multiplication by $i$, and

$$
\frac{D \tau(X)}{\partial \bar{z}}=\frac{1}{2} \tau\left(\frac{D X}{\partial x_{1}}+J \frac{D X}{\partial x_{2}}\right) .
$$

Thus if $Z=\tau(X)$, (54) follows from (16) which states that

$$
d^{2} E_{\omega}(f)(Z, \bar{Z})=4 \int_{\Sigma}\left|\frac{D Z}{\partial \bar{z}}\right|^{2} d x d y
$$


since the curvature term vanishes. The second term in (51) takes the form

$$
\begin{aligned}
-2 \int_{\Sigma}\left\{\frac { \dot { \eta } _ { 1 1 } } { \lambda ^ { 2 } } \left[\left\langle\frac{D X}{\partial x_{1}}, \frac{\partial f}{\partial x_{1}}\right\rangle\right.\right. & \left.-\left\langle\frac{D X}{\partial x_{2}}, \frac{\partial f}{\partial x_{2}}\right\rangle\right] \\
& \left.+\frac{\dot{\eta}_{12}}{\lambda^{2}}\left[\left\langle\frac{D X}{\partial x_{2}}, \frac{\partial f}{\partial x_{1}}\right\rangle+\left\langle\frac{D X}{\partial x_{1}}, \frac{\partial f}{\partial x_{2}}\right\rangle\right]\right\} d x_{1} d x_{2},
\end{aligned}
$$

which can be rewritten as

$$
-2 \int_{\Sigma} \frac{1}{\lambda^{2}}\left[\left\langle\frac{D X}{\partial x_{1}},\left(\dot{\eta}_{11}+J \dot{\eta}_{12}\right) \frac{\partial f}{\partial x_{1}}\right\rangle-\left\langle\frac{D X}{\partial x_{2}},\left(J \dot{\eta}_{11}-\dot{\eta}_{12}\right) \frac{\partial f}{\partial x_{1}}\right\rangle\right] d x_{1} d x_{2}
$$

or

$$
-2 \int_{\Sigma} \frac{1}{\lambda^{2}}\left\langle\frac{D X}{\partial x_{1}}+J \frac{D X}{\partial x_{2}},\left(\dot{\eta}_{11}+J \dot{\eta}_{12}\right) \frac{\partial f}{\partial x_{1}}\right\rangle d x_{1} d x_{2} .
$$

Finally, the third term is

$$
\int_{\Sigma}\left(\frac{\dot{\eta}_{11}^{2}}{\lambda^{4}}+\frac{\dot{\eta}_{12}^{2}}{\lambda^{4}}\right)\left\langle\frac{\partial f}{\partial x_{1}}, \frac{\partial f}{\partial x_{1}}\right\rangle d x_{1} d x_{2}
$$

Adding the three terms together yields

$$
\begin{aligned}
& d^{2} E(f, \omega)((X, \dot{\eta}),(X, \dot{\eta})) \\
& \quad=\int_{\Sigma}\left\|\frac{D X}{\partial x_{1}}+J \frac{D X}{\partial x_{2}}-\frac{\dot{\eta}_{11}}{\lambda^{2}} \frac{\partial f}{\partial x_{1}}-\frac{\dot{\eta}_{12}}{\lambda^{2}} J \frac{\partial f}{\partial x_{1}}\right\|^{2} d x_{1} d x_{2} .
\end{aligned}
$$

If $Z=\tau(X)$, we can write the right-hand side of this equation as

$$
4 \int_{\Sigma}\left\|\frac{D Z}{\partial \bar{z}}-\frac{\dot{\eta}_{11}+i \dot{\eta}_{12}}{\lambda^{2}} \frac{\partial f}{\partial z}\right\|^{2} d x_{1} d x_{2}
$$

Suppose now that we consider $d^{2} E(f, \omega)((X, \dot{\eta}),(Y, 0))$, where $X$ is a section of $\left(f^{*} T M\right)^{\top}$ and $Y$ is an arbitrary section of $f^{*} T M$. By the argument in the preceding paragraph, it then follows from (16) that the first term in (52) can be written as

$$
\int_{\Sigma}\left\langle\frac{D X}{\partial x_{1}}+J \frac{D X}{\partial x_{2}}, \frac{D Y}{\partial x_{1}}+J \frac{D Y}{\partial x_{2}}\right\rangle d x_{1} d x_{2}
$$

The second term in (52) is

$$
\begin{aligned}
-\int_{\Sigma}\left\{\frac { \dot { \eta } _ { 1 1 } } { \lambda ^ { 2 } } \left[\left\langle\frac{D Y}{\partial x_{1}}, \frac{\partial f}{\partial x_{1}}\right\rangle-\right.\right. & \left.\left\langle\frac{D Y}{\partial x_{2}}, \frac{\partial f}{\partial x_{2}}\right\rangle\right] \\
& \left.+\frac{\dot{\eta}_{12}}{\lambda^{2}}\left[\left\langle\frac{D Y}{\partial x_{2}}, \frac{\partial f}{\partial x_{1}}\right\rangle+\left\langle\frac{D Y}{\partial x_{1}}, \frac{\partial f}{\partial x_{2}}\right\rangle\right]\right\} d x_{1} d x_{2},
\end{aligned}
$$

and the third term vanishes. Thus in this case, we obtain

$$
\begin{aligned}
& d^{2} E(f, \omega)((X, \dot{\eta}),(Y, 0)) \\
& =\int_{\Sigma}\left\langle\frac{D X}{\partial x_{1}}+J \frac{D X}{\partial x_{2}}-\frac{\dot{\eta}_{11}}{\lambda^{2}} \frac{\partial f}{\partial x_{1}}-\frac{\dot{\eta}_{12}}{\lambda^{2}} \frac{\partial f}{\partial x_{2}}, \frac{D Y}{\partial x_{1}}+J \frac{D Y}{\partial x_{2}}\right\rangle d x_{1} d x_{2}
\end{aligned}
$$

Since any variation is the sum of a variation $\left(X, \dot{\eta}_{a b}\right)$, where $X$ is tangent and a variation $(Y, 0)$, it follows that the Jacobi fields $\left(X, \dot{\eta}_{a b}\right)$ for which $X$ is tangent are exactly the solutions to the equation

$$
\frac{D X}{\partial x_{1}}+J \frac{D X}{\partial x_{2}}-\frac{\dot{\eta}_{11}}{\lambda^{2}} \frac{\partial f}{\partial x_{1}}-\frac{\dot{\eta}_{12}}{\lambda^{2}} \frac{\partial f}{\partial x_{2}}=0 .
$$


We can express this in terms of sections of the line bundle $\mathbf{L}$ and holomorphic quadratic differentials as

$$
\frac{D Z}{\partial \bar{z}}-\frac{\bar{h}}{\lambda^{2}} \frac{\partial f}{\partial z}=0, \quad \text { where } \quad h d z^{2}=\left(\dot{\eta}_{11}-i \dot{\eta}_{12}\right) d z^{2}
$$

is a holomorphic quadratic differential.

Proposition 5.3. The Jacobi fields $(X, \dot{\omega})$, where $X$ is a section of $\left(f^{*} T M\right)^{\top}$, correspond to pairs $\left(Z, h d z^{2}\right)$, where $Z$ is a section of $\mathbf{L}$ and $h d z^{2}$ is a holomorphic quadratic differential which together satisfy the equation (58).

Note that

$$
\bar{\partial}(Z)=\frac{D Z}{\partial \bar{z}} d \bar{z}=\frac{\bar{h}}{\lambda^{2}} \frac{\partial f}{\partial z} d \bar{z}=\left(\frac{\bar{h}(d \bar{z})^{2}}{\lambda^{2} d z d \bar{z}}\right)\left(\frac{\partial f}{\partial z} d z\right) .
$$

The first factor within large parentheses on the right of (59) is just the extremal Beltrami differential corresponding to $h d z^{2}$ under the mapping (43). This Beltrami differential can be regarded as a section of $\mathbf{K}^{-1} \otimes \overline{\mathbf{K}}$, while multiplication by $(\partial f / \partial z) d z$ yields a holomorphic map from $\mathbf{K}^{-1}$ to $\mathbf{L}$. Thus both sides of the left equation of (59) are sections of $\mathbf{L} \otimes \overline{\mathbf{K}}$.

To estimate the size of the space of tangential Jacobi fields, we can apply the Riemann-Roch theorem to the operator $\bar{\partial}: \mathbf{L} \rightarrow \mathbf{L} \otimes \overline{\mathbf{K}}$ appearing in (59) to obtain

$$
\operatorname{dim} \mathcal{O}(\mathbf{L})-\operatorname{dim} \mathcal{O}(\overline{\mathbf{L}} \otimes \mathbf{K})=\left\langle c_{1}(\mathbf{L}),[\Sigma]\right\rangle+1-g,
$$

where $\mathcal{O}(\mathbf{L})$ is the space of holomorphic sections of $\mathbf{L}$. If the conformal harmonic map $f$ has no branch points, $\mathbf{L}=\overline{\mathbf{K}}$ and the cokernel can be identified with the space of holomorphic quadratic differentials $\mathcal{O}\left(\mathbf{K}^{2}\right)$, while the number of solutions to (59) equals the dimension of the space of holomorphic sections of the tangent bundle $\mathbf{K}^{-1} \cong \overline{\mathbf{K}}$.

Now imagine that the degree of $\mathbf{L}$ increases by one, say $\mathbf{L}=\overline{\mathbf{K}} \otimes \zeta_{p}$. Either the dimension of $\mathcal{O}(\mathbf{L})$ is one larger than the dimension of $\mathcal{O}\left(\mathbf{K}^{-1}\right)$, or the dimension of $\mathcal{O}\left(\mathbf{L}^{-1} \otimes \mathbf{K}\right)$ is one smaller than the dimension of the space of holomorphic quadratic differentials. In the latter case, since $\mathbf{L}^{-1} \otimes \mathbf{K}=\mathbf{K} \otimes \mathbf{K} \otimes \zeta_{p}^{-1}$, the dimension of the space of holomorphic quadratic differentials vanishing at $p$ to order one is one less than the space of holomorphic quadratic differentials. Of course $\mathcal{O}\left(\mathbf{K} \otimes \mathbf{K} \otimes \zeta_{p}^{-1}\right)$ is the kernel of the adjoint operator, and thus $\bar{\partial}: \mathbf{L} \rightarrow \mathbf{L} \otimes \overline{\mathbf{K}}$ must map onto a complement of the antiholomorphic sections of $\overline{\mathbf{K}} \otimes \overline{\mathbf{K}} \otimes \zeta_{p}$. Hence at least one nonzero antiholomorphic quadratic differential is covered by $\bar{\partial}$. In either case, we get a new solution to (59).

If $\Sigma$ has genus $\geq 2$, then $\operatorname{dim} \mathcal{O}(\overline{\mathbf{K}})=0$, since $\overline{\mathbf{K}}$ has negative first Chern class, and the cokernel of $\bar{\partial}$ on the holomorphic tangent bundle to $\Sigma$ is isomorphic to $\mathcal{O}(\mathbf{K} \otimes \mathbf{K})$. Thus if

$$
\begin{aligned}
& \mathcal{J}(\mathbf{L})=\left\{\text { sections } Z \text { of } \mathbf{L} \text { such that }\left(Z, h d z^{2}\right)\right. \\
& \text { solves (58), for some choice of holomorphic } \\
& \text { quadratic differential } \left.h d z^{2}=\left(\dot{\eta}_{11}-i \dot{\eta}_{12}\right) d z^{2}\right\},
\end{aligned}
$$

we must have $\mathcal{J}(\mathbf{L})=0$ when $\mathbf{L}$ is isomorphic to $\overline{\mathbf{K}}$ (no branch points). We can perform an induction using the process described in the preceding paragraph. At each stage tensoring $\mathbf{L}$ with a point bundle will either yield a new holomorphic section of $\mathbf{L}$ or a new solution to (59) with nonzero holomorphic differential, in 
either case yielding a new element of $\mathcal{J}(\mathbf{L})$. The induction yields the following lemma.

Lemma 5.1. If $\Sigma$ has genus at least two, the complex dimension of $\mathcal{J}(\mathbf{L})$ is the total branching order $\nu$ of $f$. Similarly, when $\Sigma$ has genus one the dimension of $\mathcal{J}(\mathbf{L})$ is $\nu+1$, and when $\Sigma$ has genus zero the dimension of $\mathcal{J}(\mathbf{L})$ is $\nu+3$.

Remark 5.1. Notice that an element $Z$ of $\mathcal{J}(\mathbf{L})$ determines the holomorphic quadratic differential $h d z^{2}$ such that (158) is satisfied. Thus the space $\mathcal{J}(\mathbf{L})$ is isomorphic to the space

$$
\begin{aligned}
\mathcal{J}\left(\left(f^{*} T M\right)^{\top}\right)=\left\{\text { pairs }(X, \dot{\omega}), \text { where } X \text { is a section of }\left(f^{*} T M\right)^{\top}\right. \text { and } \\
\begin{array}{r}
\dot{\omega}=\left(\dot{\eta}_{11}+i \dot{\eta}_{12}\right)\left(d \bar{z} / \lambda^{2} d z\right) \text { is an extremal } \\
\text { Beltrami differential which solve (57) }\} .
\end{array}
\end{aligned}
$$

We will use this isomorphism repeatedly in subsequent arguments.

Remark 5.2. In a neighborhood $U$ of any branch point, any element of $\mathcal{J}(\mathbf{L})$ can be divided into the sum of a meromorphic section of the holomorphic tangent bundle $\mathbf{K}^{-1}$ restricted to $U$ and a smooth bounded section of $\mathbf{K}^{-1}$ over $U$, and therefore any element of $\mathcal{J}(\mathbf{L})$ has a well-defined principal part at each branch point. An induction on the degree of $\mathbf{L}$ similar to the one described above shows that there is an element of $\mathcal{J}(\mathbf{L})$ which assumes any prescribed principal parts at the branch points of $f$, an important fact to be used later.

Remark 5.3. By conjugating the first equation in (59) and taking the inner product of the result with $(\partial f / \partial z) d z$, we obtain

$$
\left\langle\frac{D \bar{Z}}{\partial z}, \frac{\partial f}{\partial z}\right\rangle d z^{2}=\frac{1}{2} h d z^{2} .
$$

Since $Z$ determines the pair $\left(Z, h d z^{2}\right), \mathcal{J}(\mathbf{L})$ is the conjugate of the preimage of the space of holomorphic quadratic differentials under the map

$$
\bar{Z} \mapsto\left\langle\frac{D \bar{Z}}{\partial z}, \frac{\partial f}{\partial z}\right\rangle d z^{2} .
$$

\section{Minimal surfaces Without Branch points}

In 93 we proved that when a generic Riemannian metric is chosen on $M$, somewhere injective harmonic surfaces without branch points must be as nondegenerate as allowed by the group of symmetries. Now that we have the second variation formula for parametrized minimal surfaces at our disposal, it is not surprising that we can modify the proof schema presented in $\$ 3$ to give the corresponding result for minimal surfaces.

Proposition 6.1. Suppose that $M$ is a compact connected manifold of dimension at least three. For a generic choice of Riemannian metric on $M$, every prime oriented compact parametrized minimal surface $f: \Sigma \rightarrow M$ with no branch points is as nondegenerate as allowed by its connected group $G$ of symmetries. If $G$ is trivial, it is Morse nondegenerate for the two-variable energy $E$ in the usual sense. If $G=S^{1} \times S^{1}$ or $P S L(2, \mathbb{C})$, then all such minimal surfaces lie on nondegenerate critical submanifolds for $E$ which are orbits for the $G$-action. 
To prove this, we replace the Euler-Lagrange map $F$ utilized in $₫ 3$ by the corresponding map

$$
F: \operatorname{Map}(\Sigma, M) \times \mathcal{T} \times \operatorname{Met}(M) \longrightarrow \operatorname{Map}(\Sigma, T M) \times T \mathcal{T}
$$

obtained by first variation of $E$, as described in Proposition 5.1, where $\mathcal{T}$ is the Teichmüller space of $\Sigma$. This map can be divided into two components

$$
\begin{gathered}
F_{0}: \operatorname{Map}(\Sigma, M) \times \mathcal{T} \times \operatorname{Met}(M) \longrightarrow \operatorname{Map}(\Sigma, T M), \\
F_{1}: \operatorname{Map}(\Sigma, M) \times \mathcal{T} \times \operatorname{Met}(M) \longrightarrow T \mathcal{T},
\end{gathered}
$$

the first of which is the familiar tension map

$$
F_{0}(f, \omega, g)=-\frac{1}{\lambda^{2}}\left[\frac{D^{g}}{\partial x_{1}}\left(\frac{\partial f}{\partial x_{1}}\right)+\frac{D^{g}}{\partial x_{2}}\left(\frac{\partial f}{\partial x_{2}}\right)\right],
$$

where $\left(x_{1}, x_{2}\right)$ are isothermal coordinates with respect to $\omega$. In accordance with (44) and (45), the second component is simply the map

$$
F_{1}(f, \omega, g)=-P\left(\left\langle\frac{\partial f}{\partial z}, \frac{\partial f}{\partial z}\right\rangle d z^{2}\right) \in T_{\omega} \mathcal{T}
$$

where $P$ is the $L^{2}$ orthogonal projection to the space of holomorphic quadratic differentials (46). The critical points for the energy $E$ are the points $(f, \omega, g)$ such that $F(f, \omega, g)$ is a zero-section of the tangent bundle to $\operatorname{Map}(\Sigma, M) \times \mathcal{T}$.

Recall that the derivative of $F$ at a critical point $(f, \omega, g)$ for $E$ in the direction of $\operatorname{Map}(\Sigma, M) \times \mathcal{T}$, which we denote by $D_{1} F$, can be determined from the second variation formula for $E$ worked out in $\$ 5$, This determines the Jacobi operator,

$$
\left(\pi_{V} \circ D_{1} F\right)_{(f, \omega, g)}=L_{f, \omega, g}: \Gamma\left(f^{*} T M\right) \oplus T_{\omega} \mathcal{T} \rightarrow \Gamma\left(f^{*} T M\right) \oplus T_{\omega} \mathcal{T},
$$

which divides into two components $L_{0}$ and $L_{1}$, taking values in $\Gamma\left(f^{*} T M\right)$ and $T_{\omega} \mathcal{T}$ respectively.

To calculate the derivative $D_{2} F$ with respect to the metric, we also need to divide into components $D_{2} F_{0}$ and $D_{2} F_{1}$. The component $D_{2} F_{0}$ is treated by the methods of $\$ 3$. In particular, if a tangential variation $\dot{g}$ in the metric on $M$ is represented by the (not necessarily holomorphic) quadratic differential

$$
k d z^{2}=\left(\dot{g}_{11}-i \dot{g}_{22}\right) d z^{2}, \quad \text { with } \quad \dot{g}_{11}+\dot{g}_{22}=0,
$$

it follows from (28) that

$$
\int_{\Sigma}\left\langle\pi_{V} \circ\left(D_{2} F_{0}\right)_{(f, \omega, g)}(\dot{g}), Z\right\rangle d A=\int_{\Sigma} \frac{2}{\lambda^{2}}\left\langle\frac{D Z}{\partial \bar{z}}, \frac{k}{\sigma^{2}} \frac{\partial f}{\partial \bar{z}}\right\rangle d A .
$$

With these preparations in place, the proof of Proposition 6.1 follows the same pattern as that used for Proposition 3.1. We let

$$
\begin{array}{r}
\mathcal{P}_{\emptyset}=\{(f, \omega, g) \in \operatorname{Map}(\Sigma, M) \times \mathcal{T} \times \operatorname{Met}(M): f \text { is a prime conformal } \\
\omega \text {-harmonic immersion for } g \text { with no branch points }\},
\end{array}
$$

with the subscript $\emptyset$ denoting that the branch locus is empty. We note that by the discussion in $\$$, elements of $\mathcal{P}_{\emptyset}$ are automatically somewhere injective, and in fact, each element of $\mathcal{P}_{\emptyset}$ will be injective on an open dense subset of $\Sigma$.

As before, we let $\mathcal{E}$ be the subbundle of $T \operatorname{Map}(\Sigma, M)$ of finite codimension whose sections are orthogonal to the Jacobi fields forced by the $G$-action, and we let $p$ 
denote the bundle projection onto $\mathcal{E}$. To show that $\mathcal{P}_{\emptyset}$ is a submanifold, we need only show that $\left(p \circ F_{0}, F_{1}\right)$ is transverse to the zero section of the subbundle

$$
\mathcal{E} \times T \mathcal{T} \subset T(\operatorname{Map}(\Sigma, M) \times \mathcal{T}) .
$$

For a given choice of $(f, \omega, g)$ in $\mathcal{P}_{\emptyset}$, we let

$$
\begin{aligned}
\mathcal{V}=\{(X, \dot{\omega}): & X \text { is a section of the fiber of } \mathcal{E} \text { over } f, \dot{\omega} \in T_{\omega} \mathcal{T} \\
& \text { and } \left.(X, \dot{\omega}) \text { is perpendicular to the image of } \pi_{V} \circ D F_{(f, \omega, g)}\right\} .
\end{aligned}
$$

Working in a neighborhood of an injective point, we can construct local coordinates $\left(u_{1}, \ldots, u_{n}\right)$ on $M$ and isothermal coordinates $\left(x_{1}, x_{2}\right)$ on $\Sigma$, related via the exponential map on the normal bundle as in $\$ 3$ so that $u_{1} \circ f=x_{1}$ and $u_{2} \circ f=x_{2}$, $\left(u_{3}, \ldots, u_{n}\right)$ vanish on the image of $\Sigma$, and the metric coefficients for $\left(u_{1}, \ldots, u_{n}\right)$ satisfy $g_{i r}=\delta_{i r}$, for $1 \leq i \leq n$ and $3 \leq r \leq n$. Then since $f$ is somewhere injective, the first part of the argument for Lemma 3.1 shows that elements of $\mathcal{V}$ must be tangent to $\Sigma$.

We need to modify the second part of the argument to allow for variations in the Teichmüller direction. To calculate $D_{2} F_{1}$, we let

$$
\dot{\omega}=h d z^{2}=\left(\dot{\eta}_{11}-i \dot{\eta}_{12}\right) d z^{2}
$$

be a holomorphic quadratic differential and set $\dot{\eta}_{22}=-\dot{\eta}_{11}$. We then consider a smooth family of metrics $t \mapsto g(t)$, and using the inner product (44), find that

$$
\begin{aligned}
\operatorname{Re}\left\langle\left\langle F_{1}(f, \omega, g(t)), \dot{\omega}\right\rangle\right\rangle= & -\operatorname{Re}\left\langle\left\langle\Omega_{f}(t), \dot{\omega}\right\rangle\right\rangle \\
& =-\int_{\Sigma} \frac{1}{2 \lambda^{2}}\left[\dot{\eta}_{11}\left(g_{11}(t)-g_{22}(t)\right)+2 \dot{\eta}_{12} g_{12}(t)\right] d x_{1} d x_{2} .
\end{aligned}
$$

We differentiate this with respect to $t$, letting $\dot{g}$ denote the derivative of $g(t)$ at $t=0$, obtaining the result

$$
\begin{aligned}
& \operatorname{Re}\left\langle\left\langle D_{2} F_{1}(f, \omega, g)(\dot{g}), \dot{\omega}\right\rangle\right\rangle=-\int_{\Sigma} \frac{1}{2 \lambda^{2}}\left[\dot{\eta}_{11}\left(\dot{g}_{11}-\dot{g}_{22}\right)+2 \dot{\eta}_{12} \dot{g}_{12}\right] d x_{1} d x_{2} \\
& =-\int_{\Sigma} \frac{1}{2 \lambda^{2}}\left[\sum_{a, b=1}^{2} \dot{\eta}_{a b} \dot{g}_{a b}\right] d x_{1} d x_{2}=-\operatorname{Re}\left\langle\left\langle\left(\dot{g}_{11}-i \dot{g}_{12}\right) d z^{2}, h d z^{2}\right\rangle\right\rangle .
\end{aligned}
$$

From this we can infer that

$$
\left\langle\left\langle D_{2} F_{1}(f, \omega, g)(\dot{g}), \dot{\omega}\right\rangle\right\rangle=-\int_{\Sigma} \frac{2}{\sigma^{2}}\left\langle\frac{\bar{h}}{\lambda^{2}} \frac{\partial f}{\partial z}, \frac{k}{\lambda^{2}} \frac{\partial f}{\partial \bar{z}}\right\rangle d A,
$$

where

$$
k=\left(\dot{g}_{11}-i \dot{g}_{12}\right) \quad \text { and } \quad f^{*}\langle\cdot, \cdot\rangle=\sigma^{2}\left(d x_{1}^{2}+d x_{2}^{2}\right),
$$

From (64) and (67) we conclude that if $Z$ is a section of $\mathbf{L}$, then

$$
\left\langle\left\langle D_{2} F(f, \omega, g)(\dot{g}),(Z, \dot{\omega})\right\rangle\right\rangle=2 \int_{\Sigma}\left\langle\frac{D Z}{\partial \bar{z}}-\frac{\bar{h}}{\lambda^{2}} \frac{\partial f}{\partial z}, \frac{k}{\lambda^{2} \sigma^{2}} \frac{\partial f}{\partial \bar{z}}\right\rangle d A,
$$

when the variation in the metric $\dot{g}$ is represented by the quadratic differential $k d z^{2}$. Thus if $X$ is tangential to $\Sigma$, and hence the real part of a section $Z$ of $\mathbf{L}$, and $\dot{\omega}$ is an extremal Beltrami differential, then $(X, \dot{\omega})$ is perpendicular to all variations in the metric if and only if

$$
\frac{D Z}{\partial \bar{z}}=\frac{\bar{h}}{\lambda^{2}} \frac{\partial f}{\partial z}
$$


which is the condition that $(X, \dot{\omega})$ be an element of $\mathcal{J}\left(\left(f^{*} T M\right)^{\top}\right)$, the space of tangential Jacobi fields.

Thus we see that $\mathcal{V}$ consists of tangential Jacobi fields $(X, \dot{\omega})$, elements of the space $\mathcal{J}\left(\left(f^{*} T M\right)^{\top}\right)$. Here $X$ is the real part of an element $Z \in \mathcal{J}(\mathbf{L})$ and $\dot{\omega}$ corresponds to a holomorphic quadratic differential $h d z^{2}$, which together satisfy (68).

This yields the transversality needed to finish the proof of the analog of Lemma 3.1:

Lemma 6.1. If $(f, \omega)$ is a critical point for $E$ with $f$ somewhere injective, the only elements of $T_{(f, \omega)}(\operatorname{Map}(\Sigma, M) \times \mathcal{T})$ that can be perpendicular to the image of every variation in the metric are the tangential Jacobi fields $(X, \dot{\omega})$, which correspond to elements of $\mathcal{J}(\mathbf{L})$.

Up to this point we have not used the hypothesis that $f$ has no branch points. We now use it to conclude that the only tangential Jacobi fields are those generated by the $G$-action. It then follows from Lemma 6.1 that $\mathcal{V}=0$, and hence $\pi_{V} \circ D F_{(f, \omega, g)}$ maps onto the space

$$
\left\{(X, \dot{\omega}): X \text { is a section of the fiber of } \mathcal{E} \text { over } f \text { and } \dot{\omega} \in T_{\omega} \mathcal{T}\right\} .
$$

Thus $\mathcal{S}^{\prime}$ is a smooth submanifold of $\operatorname{Map}^{\prime}(\Sigma, M) \times \mathcal{T} \times \operatorname{Met}(M)$.

To complete the argument for Proposition 6.1, we need the following analog of Lemma 3.2.

Lemma 6.2. The projection on the second factor $\pi: \mathcal{P}_{\emptyset} \rightarrow \operatorname{Met}(M)$ is a Fredholm map of Fredholm index $d_{\Sigma}$, where $d_{\Sigma}$ is the real dimension of the group $G$ of automorphisms of $\Sigma$.

As in the proof of Lemma 3.2, we start by determining the tangent space to $\mathcal{P}_{\emptyset}$ at $(f, \omega, g)$, obtaining:

$$
\begin{aligned}
T_{(f, \omega, g)} \mathcal{P}_{\emptyset}=\left\{(X, \dot{\omega}, h) \in T_{f} \operatorname{Map}(\Sigma, M) \oplus T_{\omega} \mathcal{T} \oplus T_{g} \operatorname{Met}(M):\right. \\
\left.L(X, \dot{\omega})+\pi_{V} \circ\left(D_{2} F\right)_{(f, \omega, g)}(h)=0\right\},
\end{aligned}
$$

$X$ being real-valued and $L$ being the two-component Jacobi operator described in $\$ 5$. With this as a starting point, the rest of the proof of Lemma 6.2 is a straightforward modification of that given in 3 ,

Indeed, we can calculate the kernel and the range of the differential of the projection on the second factor,

We find that

$$
d \pi_{(f, \omega, g)}: T_{(f, \omega, g)} \mathcal{P}_{\emptyset} \rightarrow T_{g} \operatorname{Met}(M) .
$$

Kernel of $d \pi_{(f, \omega, g)}=\left\{(X, \dot{\omega}, h) \in T_{f} \operatorname{Map}(\Sigma, M) \oplus T_{\omega} \mathcal{T} \oplus T_{g} \operatorname{Met}(M)\right.$ :

$$
\left.h=0, L_{0}(X, \dot{\omega})=0 \text { and } L_{1}(X, \dot{\omega})=0\right\},
$$

from which we conclude that the kernel of $d \pi_{(f, \omega, g)}$ is isomorphic to the kernel of $L_{f, \omega, g}$, which is just the space of Jacobi fields for the energy $E$.

On the other hand,

$$
\begin{aligned}
& \text { Range of } d \pi_{(f, \omega, g)}=\left\{h \in T_{g} \operatorname{Met}(M):\right. \\
& \qquad \begin{array}{r}
\pi_{V} \circ\left(D_{2} F\right)_{(f, \omega, g)}(h)+L_{0}(X, \dot{\omega})=0, L_{1}(X, \dot{\omega})=0, \\
\left.\quad \text { for some }(X, \dot{\omega}) \in T_{(f, \omega)}(\operatorname{Map}(\Sigma, M) \times \mathcal{T})\right\} .
\end{array}
\end{aligned}
$$


Since variations in the metric map via $D_{2} F$ onto the $L^{2}$-orthogonal complement of the Jacobi fields, we can show, just as in 3 , that the cokernel of $d \pi_{(f, \omega, g)}$ has the same dimension as the orthogonal complement to the space $\mathcal{F}$ of Jacobi fields forced by the $G$-action in the full space of Jacobi fields. Thus the Fredholm index of $d \pi$ is the real dimension of $G$, finishing the proof of Lemma 6.2.

To finish the proof of Proposition 6.1, we simply apply the Sard-Smale Theorem as before.

Remark 6.1. We can modify Remark 3.1 by replacing $F$ by $F_{0}$ so that (25) is replaced by

$$
B(\dot{\omega}, Z)=\int_{\Sigma} D_{2}\left\langle F_{0}(f, \omega, g), Z\right\rangle(\dot{g}) d A,
$$

and once again we find that if $\dot{\omega}=h d z^{2}=\left(\dot{g}_{11}-i \dot{g}_{22}\right) d z^{2}$, then

$$
B(\dot{\omega}, Z)=-\frac{i}{2} \int_{\Sigma}\left(\frac{\partial h}{\partial \bar{z}} d z(Z)\right) d z \wedge d \bar{z}=-\frac{i}{2} \int_{\Sigma} \bar{\partial}(\dot{\omega})(Z) .
$$

We will use this definition for the lemma in $₫ 7$

\section{ELIMINATING BRANCH POINTS}

We would like to extend the argument of $₫\left[6\right.$ from the space $\mathcal{P}_{\emptyset}$ of prime minimal surfaces without branch points to the space $\mathcal{P}$ of all prime minimal surfaces. The most natural strategy for accomplishing this would be to show that the EulerLagrange map

$$
F: \operatorname{Map}(\Sigma, M) \times \mathcal{T} \times \operatorname{Met}(M) \longrightarrow T(\operatorname{Map}(\Sigma, M) \times \mathcal{T})
$$

is as transversal to the zero section of the tangent bundle at every point $(f, \omega, g)$ of $\mathcal{P}$ as allowed by the group $G$ of symmetries. By Lemma 6.1 , it would suffice to find variations $\dot{g}$ in the metric such that the image of the map

$$
\dot{g} \quad \mapsto \quad \pi_{V} \circ D_{2} F(f, \omega, g)(\dot{g})
$$

covers all the tangential Jacobi fields provided by the branch points. In this case $\mathcal{P}$ would be a submanifold, and the differential of the projection to the space $\operatorname{Met}(M)$ of metrics would fail to be surjective at minimal surfaces with branch points, implying that such minimal surfaces do not exist for generic metrics.

It is the goal of this section to construct a family of metric variations whose image under (71) covers a large portion of the space of tangential Jacobi fields (but not the entire space when $f$ has branch points of branching order $\geq 2$ ). In a very rough sense, we might think of these metric variations as "killing" the Jacobi fields, or "perturbing away" branch points on minimal surfaces.

Where might such metric variations come from? Since the Jacobi fields we want to eliminate are tangential, we expect that the variations in the metric should also be tangent to $\Sigma$. Moreover, we expect that the global geometry of the Riemann surface will be needed for their construction, since the fact that the minimal surfaces are closed must be essential to the argument.

Suppose that $f: \Sigma \rightarrow M$ is a prime parametrized minimal surface with branch points, and hence $f^{-1}(f(p))=\{p\}$, for $p$ belonging to an open dense subset of $\Sigma$ as described in Lemma 4.3. There must then be a wide variety of tangential variations in the metric on $M$, supported away from the branch points and the self-intersection set. Indeed, we can prescribe the metric variation at will except 
at the branch points and points of self-intersection. It is natural to expect that some of these will change the conformal structure induced by $f$ on the punctured Riemann surface $\Sigma-\left\{p_{1}, \ldots, p_{n}\right\}$, where $\left\{p_{1}, \ldots, p_{n}\right\}$ are the branch points. It is this change in conformal structure that we exploit in our construction of the metric variations.

Recall that a Riemann surface is said to be conformally finite if it is conformally equivalent to a compact Riemann surface with finitely many points (called punctures) removed. Suppose that $\Sigma_{0}$ is constructed from a compact oriented surface $\Sigma$ of genus $g$ by deleting $n$ points $\left\{p_{1}, \ldots, p_{n}\right\}$, where $n>0$ and $n \geq 3$ if $g=0$. Well-known arguments (34], Chapter II, as modified by passing to branched covers as in 11, Chapter II, §1) show that the Teichmüller space $\mathcal{T}_{g, n}$ of conformally finite conformal structures on $\Sigma_{0}$ is diffeomorphic to an open ball in Euclidean space of dimension $6 g-6+2 n$ so long as this number is positive. (There is a single conformal structure on the sphere minus three points.) The extra variations ( $2 n$ when $\Sigma$ has genus at least two) can be thought of as follows: If we consider a fixed conformal structure on the compact surface $\Sigma$ and allow the punctures $\left\{p_{1}, \ldots, p_{n}\right\}$ to vary, we generally obtain a family of varying conformal structures on the punctured surface. This family of conformal structures can be realized by a family of diffeomorphisms of $\Sigma$, isotopic to the identity, which do not leave given punctures fixed.

When $6 g-6+2 n \geq 0$, the space $\mathcal{T}_{g, n}$ can be described as follows: For a given divisor $D=\nu_{1} p_{1}+\cdots+\nu_{n} p_{n}$, let

$$
\operatorname{Diff}_{0, D}=\left\{\phi \in \operatorname{Diff}_{0}: \phi\left(p_{i}\right)=p_{i} \text { for each } i\right\} .
$$

Then $\mathcal{T}_{g, n}$ is defined as the quotient space

$$
\mathcal{T}_{g, n}=\operatorname{Met}_{0}\left(\Sigma_{0}\right) / \operatorname{Diff}_{0, D}(\Sigma)=\operatorname{Conf}\left(\Sigma_{0}\right) / \operatorname{Diff}_{0, D}(\Sigma) .
$$

The larger group $\operatorname{Diff}_{0}(\Sigma)$, which allows the branch points to move, does not preserve the conformal structure on the punctured surface.

Each conformal equivalence class in $\mathcal{T}_{g, n}$ has a unique complete Riemannian metric of constant negative curvature and total area one, the punctures being infinitely far away in terms of the metric. It is well known ([1], Chapter 2, 1 , or [7]) that the cotangent space to $\mathcal{T}_{g, n}$ consists of meromorphic quadratic differentials on the compact surface $\Sigma$ which have the possibility of simple poles only at the punctures.

The preceding discussion indicates that we should consider meromorphic quadratic differentials on $\Sigma$ with poles at the branch points when constructing the tangential variations.

For the actual construction of the tangential variations, we utilize a canonical form for a minimal surface in the neighborhood of a branch point similar to those described at the beginning of \$4. Recall that by (30), if $p$ is a branch point for $f$, then there are normal coordinates $\left(u_{1}, \ldots, u_{n}\right)$ centered at $f(p)$ on the ambient Riemannian manifold $M$ such that in terms of a complex parameter $z$ on $\Sigma$,

$$
\left(u_{1}+i u_{2}\right)(z)=c z^{\nu+1}+o_{1}\left(|z|^{\nu+1}\right), \quad u_{r}(z)=o_{1}\left(|z|^{\nu+1}\right) \quad \text { for } \quad 3 \leq r \leq n
$$

where $c$ is a nonzero complex constant. As mentioned before, substantial refinements of this normal form are presented in [27]. Indeed, it follows from Theorem 1.3 of [27] that if we set $U=\left(u_{1}, \ldots, u_{n}\right)$, then

$$
U(z)=H_{\nu+1}(z)+H_{\nu+2}(z)+o_{2}\left(|z|^{\nu+2}\right),
$$


where $H_{\nu+1}$ and $H_{\nu+2}$ are harmonic polynomials, and hence (following the first part of the proof of Theorem 1.4 of [27]) of the form

$$
H_{\nu+k}(z)=\beta_{\nu+k} z^{\nu+k}+\bar{\beta}_{\nu+k} \bar{z}^{\nu+k}, \quad \text { for } k=1,2,
$$

where $\beta_{\nu+k}$ is an element of $\mathbb{C}^{n}$. Since the coordinates $\left(u_{1}, \ldots, u_{n}\right)$ are normal and hence the Christoffel symbols vanish at $p$,

$$
\left\langle\frac{\partial f}{\partial z}, \frac{\partial f}{\partial z}\right\rangle=0 \Rightarrow \frac{\partial U}{\partial z} \cdot \frac{\partial U}{\partial z}=o_{1}\left(|z|^{2 \nu+1}\right)
$$

and hence

$$
(\nu+1)^{2} \beta_{\nu+1} \cdot \beta_{\nu+1} z^{2 \nu}+(\nu+1)(\nu+2) \beta_{\nu+1} \cdot \beta_{\nu+2} z^{2 \nu+1}=o_{1}\left(|z|^{2 \nu+1}\right),
$$

it follows that

$$
\beta_{\nu+1} \cdot \beta_{\nu+1}=0=\beta_{\nu+1} \cdot \beta_{\nu+2} .
$$

The first of these conditions implies that we can improve the first part of the canonical form to

$$
\left(u_{1}+i u_{2}\right)(z)=z^{\nu+1}+c_{1} z^{\nu+2}+c_{2} \bar{z}^{\nu+2}+o_{2}\left(|z|^{\nu+2}\right),
$$

while the second shows that we can improve it to

$$
\left(u_{1}+i u_{2}\right)(z)=z^{\nu+1}+c z^{\nu+2}+o_{2}\left(|z|^{\nu+2}\right) .
$$

This implies that if we set $w=u_{1}+i u_{2}$, then

$$
d w=\left[(\nu+1) z^{\nu+1}+c(\nu+2) z^{\nu+1}+o_{1}\left(\left|z^{\nu+1}\right|\right)\right] d z+o_{1}\left(|z|^{\nu+1}\right) d \bar{z},
$$

from which by a straightforward calculation, we derive the formula

$$
\frac{\partial}{\partial w}=\left[\frac{1}{(\nu+1) z^{\nu}}+\frac{a}{z^{\nu-1}}+o_{1}\left(|z|^{-\nu+1}\right)\right] \frac{\partial}{\partial z}+o_{1}\left(|z|^{-\nu+1}\right) \frac{\partial}{\partial \bar{z}},
$$

where $a$ is a complex constant, an important estimate to be used later.

As in the proof of Proposition 6.1, our goal is to investigate the space $\mathcal{V}$ defined by (66):

$\mathcal{V}=\left\{(X, \dot{\omega}): X\right.$ is a section of $f^{*} T M, \dot{\omega} \in T_{\omega} \mathcal{T}$ and $(X, \dot{\omega})$ is perpendicular to the image of $\left.\pi_{V} \circ D F_{(f, \omega, g)}\right\}$,

and we know that the derivative $\pi_{V} \circ D_{1} F_{(f, \omega, g)}$ with respect to the variable $(f, \omega)$ covers a complement to the space $\mathcal{J}\left(\left(f^{*} T M\right)^{\top}\right)$ of tangential Jacobi fields $(X, \dot{\omega})$, $X$ being the real part of an element of $\mathcal{J}(\mathbf{L})$, a subspace of $\Gamma(\mathbf{L})$. Recall that $F$ divides into two components, $F_{0}$ and $F_{1}$, with values in $T \operatorname{Map}(\Sigma, M)$ and $T \mathcal{T}$ respectively, where $F_{0}$ is the familiar map (63) such that $F_{0}(f, \omega, g)=0$ is the equation that $f$ be harmonic with respect to $\omega$ and $g$. It follows from Lemma 6.1 that it suffices to find new variations $\dot{g}$ in the metric which make

$$
B(\dot{\omega}, Z)=\int_{\Sigma} D_{2}\left\langle F_{0}(f, \omega, g), Z\right\rangle(\dot{g}) d A \neq 0,
$$

for appropriate elements $\left(Z, h d z^{2}\right)$ of $\mathcal{J}(\mathbf{L})$ (see Remark 6.1).

Recall from $\$ 3$ that in terms of the complex coordinate $z=x_{1}+i x_{2}$,

$$
\pi_{V} \circ\left(D_{2} F_{0}\right)_{(f, \omega, g)}(h)=-\frac{1}{\lambda^{2}} \sum_{i, j, k=1}^{n} g^{k l} \dot{\Gamma}_{l, i j}\left(\frac{\partial u_{i}}{\partial x_{1}} \frac{\partial u_{j}}{\partial x_{1}}+\frac{\partial u_{i}}{\partial x_{2}} \frac{\partial u_{j}}{\partial x_{2}}\right) \frac{\partial}{\partial x_{k}} .
$$


If $z$ is centered at the branch point $p$, the canonical form implies that

$$
\left(\frac{\partial u_{i}}{\partial x_{1}} \frac{\partial u_{j}}{\partial x_{1}}+\frac{\partial u_{i}}{\partial x_{2}} \frac{\partial u_{j}}{\partial x_{2}}\right)= \begin{cases}(\nu+1)^{2} r^{2 \nu}+o\left(r^{2 \nu}\right) & \text { if } i=j=1 \text { or } i=j=2, \\ o\left(r^{2 \nu}\right) & \text { otherwise, }\end{cases}
$$

where $r=|z|$, and hence

$$
\begin{aligned}
\left\langle\pi_{V} \circ\left(D_{2} F\right)_{(f, g)}\right. & \left.(h), \sum f^{i} \frac{\partial}{\partial x_{i}}\right\rangle d A \\
& =-\sum_{k=1}^{2}\left((\nu+1)^{2} r^{2 \nu}\left(\dot{\Gamma}_{k, 11}+\dot{\Gamma}_{k, 22}\right)+o\left(r^{2 \nu}\right)\right) f^{k} d x_{1} d x_{2} .
\end{aligned}
$$

We want to perturb away sections $Z$ which lie in $\mathcal{J}(\mathbf{L})$. We can write any such section as

$$
Z=\zeta(z) \frac{\partial f}{\partial z}, \quad \text { where } \quad \zeta(z)=a_{-\nu} \frac{1}{z^{\nu}}+\cdots+a_{-1} \frac{1}{z}+\text { (regular function), }
$$

with the $a_{i}$ 's being complex constants. We say that

$$
\text { (the principal part of } \zeta)=a_{-\nu} \frac{1}{z^{\nu}}+\cdots+a_{-1} \frac{1}{z} .
$$

Since $\partial f / \partial z$ vanishes to order $\nu$ at $p, Z(p)$ is nonzero as an element of the fiber of $\mathbf{L}$ at $p$ precisely when $a_{-\nu} \neq 0$. Recall that by Remark 5.2, any combination of principal parts at the branch points can be realized by a section $Z$ of $\mathbf{L}$, the first component of an element $\left(Z, h d z^{2}\right) \in \mathcal{J}(\mathbf{L})$.

For the statement of the next lemma, we let $\mathcal{Q}(\Sigma)$ denote the space of all quadratic differentials on $\Sigma$, and $\mathcal{H}(\Sigma)$ the subspace of holomorphic quadratic differentials.

Lemma 7.1. Suppose that $f: \Sigma \rightarrow M$ is a weakly conformal harmonic map with divisor of branch points

$$
D(f)=\lambda_{1} p_{1}+\cdots+\lambda_{n} p_{n}
$$

and that $f$ is one-to-one except for finitely many points and a subset of a finite number of $C^{1}$ curves of self-intersection of finite length. Then there is a collection $Z_{1}, \ldots, Z_{n}$ of elements of $\mathcal{J}(\mathbf{L})$ and a collection $h_{1} d z^{2}, \ldots, h_{n} d z^{2}$ of quadratic differentials on $\Sigma$, with linearly independent projections in $\mathcal{Q}(\Sigma) / \mathcal{H}(\Sigma)$, such that

$$
B\left(h_{j} d z^{2}, Z_{k}\right)=\delta_{j k}= \begin{cases}1, & \text { if } j=k, \\ 0, & \text { if } j \neq k,\end{cases}
$$

where $B$ is defined by (69). Moreover, if $\Sigma=S^{2}$, we can choose $h_{j} d z^{2}$ and $Z_{k}$ so that $Z_{1}, \ldots, Z_{n}$ vanish at three prescribed points not belonging to the branch locus, while if $\Sigma=T^{2}$, we can arrange that $Z_{1}, \ldots, Z_{n}$ vanish at one prescribed point which is not in the branch locus.

Remark 7.1. For a prime harmonic map, the hypothesis on the self-intersection set is guaranteed by Lemma 4.3. As we will see in $\$ 8$ and $\$ 10$, the above lemma implies that when a prime parametrized minimal surface $f$ has $n$ branch points, it possesses a real $(2 n)$-dimensional space of tangential Jacobi fields $(X, \dot{\omega})$ which can be eliminated by a real $(2 n)$-dimensional space of nonholomorphic quadratic differentials corresponding to tangential variations in the metric. In particular, if $f$ 
has only simple branch points, all the tangential Jacobi fields perpendicular to the $G$-orbits can be covered by metric variations.

Part I of the proof of Lemma 7.1. We first prove the lemma under the assumptions $\Sigma$ has genus at least two and $f$ has no points of self-intersection.

We begin by choosing one of the branch points $p_{j}$ and constructing a meromorphic quadratic differential $h_{j}^{\prime} d z^{2}$ with a pole of order one at $p_{j}$ and no other poles. (The existence of such a meromorphic quadratic differential, under the assumption that $\Sigma$ has genus at least two, follows from the Riemann-Roch theorem.) We will determine the nonzero coefficient of the highest order term in the principal part later.

We need to multiply this quadratic differential by a cutoff function near the poles so that it determines a well-defined deformation of the metric. To do this, we first choose a local conformal coordinate $z=x_{1}+i x_{2}$ centered at a given branch point $p_{i}$ and let $r=\sqrt{x_{1}^{2}+x_{2}^{2}}$ so that we can define the $\epsilon$-ball $B_{\epsilon}\left(p_{i}\right)$ about $p_{i}$ as the set of points satisfying $r \leq \epsilon$, for a small $\epsilon, 0<\epsilon<1$. We construct a smooth cutoff function $\phi: \Sigma \rightarrow[0,1]$ which is identically one outside the $\epsilon$-ball about each $p_{i}$, identically zero on an $\epsilon^{2}$-ball about $p_{i}$, and depends only on the radial coordinate $r$ on each of the remaining annuli within $\Sigma$.

We then let $h_{j}=\phi h_{j}^{\prime}$, thereby obtaining a new quadratic differential $h_{j} d z^{2}$ which is holomorphic except in small neighborhoods of the branch points. Since we are assuming that $f$ is an imbedding on the support of $\phi$, the quadratic differential $h_{j} d z^{2}$ can indeed be realized by a deformation of the metric on the ambient manifold $M$. Locally, such a deformation is defined by (22), when $h_{j} d z^{2}=(M-i N) d z^{2}$.

We can also choose a branch point $p_{k}$ and a section $Z_{k} \in \mathcal{J}(\mathbf{L})$ so that if $z_{i}$ is the complex coordinate centered at $p_{i}$, then near $p_{i}$,

$$
\begin{aligned}
& Z_{k}=\zeta_{k i}(z) \frac{\partial f}{\partial z_{i}}, \\
& \text { where } \zeta_{k i}(z)= \begin{cases}\left(1 / z_{i}^{\nu_{i}}\right)+\left(\text { function regular at } p_{i}\right), & \text { if } i=k, \\
\left(\text { function regular at } p_{i}\right), & \text { if } i \neq k,\end{cases}
\end{aligned}
$$

where $\nu_{i}$ is the order of the branch point $p_{i}$. It follows from (70) that the only contributions to

$$
B\left(h_{j} d z^{2}, Z_{k}\right)=\int_{\Sigma}\left\langle\pi_{V} \circ\left(D_{2} F_{0}\right)_{(f, \omega, g)}\left(h_{j}\right), Z_{k}\right\rangle d A
$$

come from the small neighborhoods of the branch points on which the derivative of the cutoff function $\phi$ is nonzero, and in fact we will see that the only contribution that does not approach zero as $\epsilon \rightarrow 0$ comes from $p_{j}$, since this is where $h_{j}^{\prime} d z^{2}$ is blowing up.

We focus on a neighborhood of $p_{j}$ and write $h_{j}^{\prime}=\left(M^{\prime}-i N^{\prime}\right)$ as in $₫ 3$. In terms of the coordinate $z$ centered at $p_{j}$ on $\Sigma$ and the corresponding coordinate $w=u_{1}+i u_{2}$ on $M$ which approximates $z^{\nu+1}$, we can apply (72), or rather, its conjugate. Since 
$h^{\prime} d z^{2}$ is a holomorphic differential, we find that

$$
\begin{aligned}
\frac{\partial}{\partial \bar{w}}( & \left.M^{\prime}-i N^{\prime}\right) \\
=\left[\left(\frac{1}{(\nu+1) \bar{z}^{\nu}}+\frac{a}{\bar{z}^{\nu-1}}+o\left(|z|^{-\nu+1}\right)\right) \frac{\partial}{\partial \bar{z}}+o\left(|z|^{-\nu+1}\right) \frac{\partial}{\partial z}\right] & \left(M^{\prime}-i N^{\prime}\right) \\
& =o\left(|z|^{-\nu-1}\right),
\end{aligned}
$$

where the extra factor $|z|^{-2}$ comes from the fact that $\left(M^{\prime}-i N^{\prime}\right)=b / z+o\left(|z|^{-1}\right)$ for some complex constant $b$, while its derivative with respect to $z$ is $-b / z^{2}+o\left(|z|^{-2}\right)$. Hence

$$
\frac{1}{2}\left[\left(\frac{\partial M^{\prime}}{\partial u_{1}}+\frac{\partial N^{\prime}}{\partial u_{2}}\right)-i\left(\frac{\partial N^{\prime}}{\partial u_{1}}-\frac{\partial M^{\prime}}{\partial u_{2}}\right)\right]=o\left(|z|^{-\nu-1}\right) .
$$

But we are interested in the variation defined by $h=\phi h^{\prime}=M-i N=\phi M^{\prime}-$ $i \phi N^{\prime}$, and in this case the calculation yields

$$
\begin{aligned}
\frac{\partial}{\partial \bar{w}}(M-i N)=\left(M^{\prime}-i N^{\prime}\right) & \frac{1}{(\nu+1) \bar{z}^{\nu}} \frac{\partial \phi}{\partial \bar{z}}+\phi(r) o\left(|z|^{-\nu-1}\right) \\
& =\left(M^{\prime}-i N^{\prime}\right) \phi^{\prime}(r) \frac{1}{(\nu+1) \bar{z}^{\nu}} \frac{\partial r}{\partial \bar{z}}+\phi(r) o\left(|z|^{-\nu-1}\right) .
\end{aligned}
$$

In terms of polar coordinates $z=r e^{i \theta}$,

$$
\begin{aligned}
\frac{\partial r}{\partial \bar{z}}=\frac{1}{2}\left(\frac{\partial}{\partial x_{1}}+i \frac{\partial}{\partial x_{2}}\right)\left(\sqrt{x_{1}^{2}+x_{2}^{2}}\right) & \\
& =\frac{1}{2}\left(\frac{x}{r}+i \frac{y}{r}\right)=\frac{1}{2}(\cos \theta+i \sin \theta)=\frac{1}{2} e^{i \theta},
\end{aligned}
$$

while

$$
\left(M^{\prime}-i N^{\prime}\right)=b \frac{1}{z}+O(1)=\frac{b e^{-i \theta}}{r}+O(1)
$$

where $b$ is a nonzero complex constant. Hence

$$
\frac{1}{2}\left[\left(\frac{\partial M}{\partial u_{1}}+\frac{\partial N}{\partial u_{2}}\right)-i\left(\frac{\partial N}{\partial u_{1}}-\frac{\partial M}{\partial u_{2}}\right)\right]=\frac{b \phi^{\prime}(r)}{2 r} \frac{1}{(\nu+1) \bar{z}^{\nu}}+\phi(r) o\left(|z|^{-\nu-1}\right),
$$

or equivalently,

$$
\left(\dot{\Gamma}_{1,11}+\dot{\Gamma}_{1,22}\right)-i\left(\dot{\Gamma}_{2,11}+\dot{\Gamma}_{2,22}\right)=\frac{b \phi^{\prime}(r)}{r} \frac{1}{(\nu+1) \bar{z}^{\nu}}+\phi(r) o\left(|z|^{-\nu-1}\right) .
$$

It follows that

$$
\begin{aligned}
& {\left[\sum_{a=1}^{2}\left(\dot{\Gamma}_{1, a a}-i \dot{\Gamma}_{2, a a}\right)\left(\zeta_{j k}(z)\right)\right]} \\
& =\frac{b \phi^{\prime}(r)}{r} \frac{1}{(\nu+1) \bar{z}^{\nu}} \zeta_{j k}(z)+\phi(r) o\left(|z|^{-2 \nu-1}\right) \\
& \quad=\frac{b \phi^{\prime}(r)}{r} \frac{1}{(\nu+1) \bar{z}^{\nu}} \zeta_{j k}(z)+\phi(r) o\left(|z|^{-2 \nu-1}\right),
\end{aligned}
$$


an equation which can be regarded as complex linear in both $\zeta$ and $b$. The real part of the last expression is the integrand in (73), so substitution into (73) yields

$$
\begin{aligned}
\int_{B_{\epsilon}\left(p_{j}\right)}\left\langle\pi_{V} \circ\left(D_{2} F\right)_{(f, \omega, g)}\left(h_{j}\right), Z_{k}\right\rangle d A & \\
=-(\nu+1) \int_{B_{\epsilon}\left(p_{j}\right)} & {\left[\left(\delta_{j k} b\right) \frac{\phi^{\prime}(r)}{r} r+\phi(r) o(1)\right] d r d \theta } \\
= & -(\nu+1) \int_{B_{\epsilon}\left(p_{j}\right)}\left(\delta_{j k} b\right) \frac{\phi^{\prime}(r)}{r} r d r d \theta+\operatorname{Error}(\epsilon),
\end{aligned}
$$

an expression linear in both $h$ and $Z$, where $\operatorname{Error}(\epsilon) \rightarrow 0$ as $\epsilon \rightarrow 0$. Thus

$$
\begin{aligned}
\int_{0}^{\epsilon} \phi^{\prime}(r) d r=\phi(\epsilon)-\phi(0)=1 & \Rightarrow \\
& \lim _{\epsilon \rightarrow 0} \int_{B_{\epsilon}\left(p_{j}\right)}\left\langle\pi_{V} \circ\left(D_{2} F_{0}\right)_{(f, \omega, g)}\left(h_{j}\right), Z_{k}\right\rangle d A=-2 \pi \delta_{j k}(\nu+1) b .
\end{aligned}
$$

Finally, we choose $b$ so that $2 \pi(\nu+1) b=-1$. A similar calculation shows that

$$
\lim _{\epsilon \rightarrow 0} \int_{B_{\epsilon}\left(p_{i}\right)}\left\langle\pi_{V} \circ\left(D_{2} F_{0}\right)_{(f, \omega, g)}\left(h_{j}\right), Z_{k}\right\rangle d A=0, \quad \text { when } i \neq j .
$$

These calculations show that our collection $Z_{1}, \ldots, Z_{n}$ of holomorphic sections of $\mathbf{L}$ and our collection $h_{1} d z^{2}, \ldots, h_{n} d z^{2}$ of quadratic differentials approximate (175) better and better as $\epsilon \rightarrow 0$. Choosing $\epsilon>0$ sufficiently small and replacing $Z_{1}, \ldots, Z_{n}$ by linear combinations of $Z_{1}, \ldots, Z_{n}$ enables us to establish (75) on the nose.

Part II of the proof of Lemma 7.1. We next treat the case that $\Sigma$ has genus zero or one and $f$ has no points of self-intersection.

If $\Sigma=S^{2}$, we introduce three additional points $\{q, r, s\}$ not in the branch locus and allow the meromorphic differential $h_{j}^{\prime} d z^{2}$ to have simple poles at these points. We must then replace each $Z_{k}$ by $Z_{k}^{\prime}=Z_{k}+W_{k}$, where $W_{k}$ is a forced Jacobi field chosen so that $Z_{k}^{\prime}(q), Z_{k}^{\prime}(r)$ and $Z_{k}^{\prime}(s)$ all vanish. We multiply by cutoff functions near each of the points $q, r$ and $s$ and carry through the calculation we presented in Part I. Assuming that $h_{j}^{\prime} d z^{2}$ is not regular, but has a simple pole at $q$, the calculation yields a zero contribution at $q$ since $Z_{k}^{\prime}(q)=0$. If it should happen that $h_{j}^{\prime} d z^{2}$ is regular at $q$, the integrand goes to zero at $q$, so a much simpler cutoff argument can be applied. Since $Z_{k}^{\prime}(r)$ and $Z_{k}^{\prime}(s)$ also vanish, we find that the contributions at $r$ and $s$ are also zero.

When $\Sigma=T^{2}$ the argument is similar, except we need only let the meromorphic differential $h^{\prime} d z^{2}$ have an additional simple pole at a single predetermined point $q$. Once again, we replace each $Z_{k}$ by $Z_{k}^{\prime}=Z_{k}+W_{k}$, where $W_{k}$ is a forced Jacobi field chosen so that $Z_{k}^{\prime}(q)=0$, and find that this forces the contribution at $q$ to be zero.

Part III of the proof of Lemma 7.1. Of course, if $f$ has self-intersections, these might interfere with realizing the quadratic differential $h_{j} d z^{2}$ by a deformation of the metric on the ambient manifold $M$. (In the overall argument, it is here that we use the special nature of the self-intersection set $K$ for prime minimal surfaces $f: \Sigma \rightarrow M$, as established by Lemma 4.3.) 
Isolated points of self-intersection, as well as the finite number of points where $C^{1}$ curves within $K$ intersect, can be treated by exactly the same procedure (multiplying by a suitable cutoff function) that we used to treat branch points at which $Z_{k}$ is regular. When these points are excised from $K$ we are left with a self-intersection set which lies within a finite number of $C^{1}$ arcs of finite length.

Therefore it only remains to check that along one of these arcs, a $C^{1}$ curve $C$ of self-intersection, we can multiply by a cutoff function $\psi: \Sigma \rightarrow[0,1]$ which vanishes on $C$ but alters (76) by an arbitrarily small amount. In a tubular neighborhood $U$ of $C$, we can choose a local coordinate system $(s, t)$ such that $s$ is arclength along $C$ and $|t|$ measures distance from $C$ in the metric on $\Sigma$ (so $C$ is defined by $t=0$ ). We then choose a $C^{1}$ function $\psi(t)$ with values in $[0,1]$, depending only on $t$, which is one when $|t| \geq \epsilon$ and zero for $|t| \leq \epsilon / 2$, and consider the quadratic differential $\psi h_{j} d z^{2}$ near $C$.

In terms of a complex parameter on $\Sigma$ near part of $C$, we find that

$$
\frac{\partial}{\partial \bar{z}}\left(\psi h_{j}\right)(s, t)=\psi^{\prime}(t) \frac{\partial t}{\partial \bar{z}}\left(M_{j}-i N_{j}\right)
$$

since $h_{j} d z^{2}$ is holomorphic along $C$. The change in the integral (76) near $C$ is approximately

$$
\left[\int_{-\epsilon}^{\epsilon} \psi^{\prime}(t) d t\right]\left[\int\left(\frac{\partial t}{\partial \bar{z}}\left(M_{j}-i N_{j}\right)\left(\zeta_{k}\right)\right) d s\right]
$$

which vanishes modulo terms of lower order, since the first factor is just $\psi(\epsilon)-$ $\psi(-\epsilon)=0$.

Thus we can replace the differential $h_{j} d z^{2}$ with its product with a cutoff function depending on $\epsilon>0$, so that the matrix whose components are given by (76) is nonsingular when $\epsilon$ is sufficiently close to zero. Once again, replacing $Z_{1}, \ldots, Z_{n}$ by linear combinations enables us to establish (75) exactly. This finishes the proof of the lemma.

\section{Simple BRANCH POINTS}

We now have the means to prove the Main Theorem for prime minimal surfaces with at worst only simple branch points. This will follow from Proposition 6.1 and

Proposition 8.1. Suppose that $M$ is a compact connected manifold of dimension at least three. For a generic choice of Riemannian metric on $M$, there are no prime minimal surfaces that have nontrivial branch points, all of which are simple.

Once again we use the pattern of proof adopted in $₫ 3$. We replace the space $\mathcal{P}_{\emptyset}$ defined by (65) and used in the proof of Proposition 6.1, by

$$
\begin{aligned}
& \mathcal{P}_{s}=\{(f, \omega, g) \in \operatorname{Map}(\Sigma, M) \times \mathcal{T} \times \operatorname{Met}(M): \\
& f \text { is a prime conformal } \omega \text {-harmonic map } \\
&\text { for } g \text { which has only simple branch points }\},
\end{aligned}
$$

an open subset of the space of all prime harmonic maps. As before, we study $\mathcal{P}_{s}$ by means of the Euler-Lagrange map

$$
F: \operatorname{Map}(\Sigma, M) \times \mathcal{T} \times \operatorname{Met}(M) \longrightarrow \operatorname{Map}(\Sigma, T M) \times T \mathcal{T},
$$

obtained by first variation of $E$. As we learned in $\$ 5$, $F$ can be divided into two components $F_{0}$ and $F_{1}$ which take values in the two factors, the first component 
being

$$
F_{0}(f, \omega, g)=-\frac{1}{\lambda^{2}}\left[\frac{D^{g}}{\partial x_{1}}\left(\frac{\partial f}{\partial x_{1}}\right)+\frac{D^{g}}{\partial x_{2}}\left(\frac{\partial f}{\partial x_{2}}\right)\right],
$$

where $\left(x_{1}, x_{2}\right)$ are isothermal coordinates with respect to $\omega$, the second being

$$
F_{1}(f, \omega, g)=-P\left(\left\langle\frac{\partial f}{\partial z}, \frac{\partial f}{\partial z}\right\rangle d z^{2}\right) \in T_{\omega} \mathcal{T},
$$

where $P$ is the orthogonal projection into the space of holomorphic quadratic differentials for $(f, \omega)$. The space $\mathcal{P}_{s}$ consists of critical points for the energy $E$, which are exactly the points $(f, \omega, g)$ such that $F(f, \omega, g)$ is a zero-section.

Once again our overall strategy is to show that $F$ is as transversal as possible to the space of zero sections. However, when $\Sigma$ has genus zero or one, we do not project into a subbundle $\mathcal{E}$ of finite codimension complementary to the space of forced Jacobi fields, but utilize instead another approach, which although slightly more complicated, fits in better with Lemma 7.1.

Suppose first that $\Sigma$ is the two-sphere. If $N$ is a compact codimension two submanifold of $M$ with boundary $\partial N$, we let

$$
\begin{aligned}
\mathcal{U}(N)=\left\{f \in \operatorname{Map}\left(S^{2}, M\right): f \text { does not intersect } \partial N,\right. \text { has nonempty } \\
\text { transversal intersection with the interior of } N, \text { and } \\
\text { in particular has no branch points which lie within } N\},
\end{aligned}
$$

which is an open subset of $\operatorname{Map}\left(S^{2}, M\right)$. Given three disjoint compact codimension two submanifolds with boundary, say $Q, R$ and $S$, we let

$$
\mathcal{U}(Q, R, S)=\mathcal{U}(Q) \cap \mathcal{U}(R) \cap \mathcal{U}(S),
$$

also an open subset of $\operatorname{Map}\left(S^{2}, M\right)$.

Next we cover $\operatorname{Map}\left(S^{2}, M\right)$ with a countable collection of sets $\mathcal{U}\left(Q_{i}, R_{i}, S_{i}\right)$ defined by a sequence $i \mapsto\left(Q_{i}, R_{i}, S_{i}\right)$ of triples of such codimension two submanifolds. We choose three points $q, r$ and $s$ in $S^{2}$ and let

$$
\mathcal{F}_{i}\left(S^{2}, M\right)=\left\{f \in \mathcal{U}\left(Q_{i}, R_{i}, S_{i}\right): f(q) \in Q_{i}, f(r) \in R_{i}, f(s) \in S_{i}\right\},
$$

noting that $\mathcal{F}_{i}\left(S^{2}, M\right)$ meets each $\operatorname{PSL}(2, \mathbb{C})$-orbit in $\mathcal{U}\left(Q_{i}, R_{i}, S_{i}\right)$ in a finite number of points. It follows from the Sobolev imbedding theorem and smoothness of the evaluation map on the space of $C^{k-2}$ maps (Proposition 2.4.17 of [4]) that the evaluation map

$$
\text { ev : } L_{k}^{2}\left(S^{2}, M\right) \times S^{2} \longrightarrow M, \quad \text { defined by } \quad \operatorname{ev}(f, p)=f(p) \text {, }
$$

is $C^{k-2}$. Thus when $k$ is large, we can regard $\mathcal{F}_{i}\left(S^{2}, M\right)$ as a submanifold of $\operatorname{Map}\left(S^{2}, M\right)$ of codimension six with tangent space

$$
\begin{aligned}
& T_{f} \mathcal{F}_{i}\left(S^{2}, M\right)=\left\{\text { sections } X \text { of } f^{*} T M:\right. \\
& \left.\qquad X(q) \in T_{f(q)} Q_{i}, X(r) \in T_{f(r)} R_{i}, X(s) \in T_{f(s)} S_{i}\right\} .
\end{aligned}
$$

Finally, we let

$$
\mathcal{A}_{i}\left(S^{2}, M\right)=\mathcal{F}_{i}\left(S^{2}, M\right) \times \operatorname{Met}(M), \quad \mathcal{P}_{s, i}=\mathcal{P}_{s} \cap \mathcal{A}_{i}\left(S^{2}, M\right) .
$$

By abuse of language, we denote the restriction of $F$ to $\mathcal{A}_{i}\left(S^{2}, M\right)$ by $F$ once again, and note that we have now broken the $P S L(2, \mathbb{C})$ symmetry.

In the case where $\Sigma$ is the torus, we once again have a nontrivial group of symmetries. This time, however, we need fix only one point to break the symmetry. 
We define $\mathcal{U}(N)$ by (78) with $S^{2}$ replaced by $T^{2}$ and choose a sequence $i \mapsto N_{i}$ of smooth compact codimension two submanifolds of $M$ such that $\mathcal{U}\left(N_{i}\right)$ cover $\operatorname{Map}\left(T^{2}, M\right)$. We choose a base point $q \in T^{2}$ and let

$$
\mathcal{F}_{i}\left(T^{2}, M\right)=\left\{f \in \mathcal{U}\left(N_{i}\right): f(q) \in N_{i}\right\} .
$$

As before, we let

$$
\mathcal{A}_{i}\left(T^{2}, M\right)=\mathcal{F}_{i}\left(T^{2}, M\right) \times \mathcal{T} \times \operatorname{Met}(M), \quad \mathcal{P}_{s, i}=\mathcal{P}_{s} \cap \mathcal{A}_{i}\left(T^{2}, M\right),
$$

with $\mathcal{T}$ being the Teichmüller space of $T^{2}$, and restrict $F$ to $\mathcal{A}_{i}\left(T^{2}, M\right)$.

If $(f, \omega, g)$ is an element of $\mathcal{P}_{s, i}$, when $\Sigma$ has genus zero or one, then just as in $\S 6$ we let

$$
\begin{aligned}
\mathcal{V}_{i}=\left\{(X, \dot{\omega}): X \in T \mathcal{F}_{i}(\Sigma, M), \dot{\omega} \in T_{\omega} \mathcal{T} \text { and }(X, \dot{\omega})\right. \\
\text { is perpendicular to the image of } \left.\pi_{V} \circ D F_{(f, \omega, g)}\right\} .
\end{aligned}
$$

If $(f, \omega, g)$ is an element of $\mathcal{P}_{s}$, when $\Sigma$ has genus at least two, we define $\mathcal{V}$ in the same way, except that $T \mathcal{F}_{i}(\Sigma, M)$ is replaced by $T \operatorname{Map}(\Sigma, M)$, the index $i$ being unnecessary in this case.

Recall that the derivative $D_{1} F$ of $F$ at a critical point $(f, \omega, g)$ for $E$ in the direction of $\operatorname{Map}(\Sigma, M) \times \mathcal{T}$ can be determined from the second variation formula for $E$ as in $\S 5$, a calculation which yields the Jacobi operator

$$
\left(\pi_{V} \circ D_{1} F\right)_{(f, \omega, g)}=L_{f, \omega, g}: T_{(f, \omega)}(\operatorname{Map}(\Sigma, M) \times \mathcal{T}) \rightarrow T_{(f, \omega)}(\operatorname{Map}(\Sigma, M) \times \mathcal{T}) .
$$

Just like $F$, this operator divides into two components $L_{0}$ and $L_{1}$, which take values in $T_{f} \operatorname{Map}(\Sigma, M)$ and $T_{\omega} \mathcal{T}$ respectively. If $(X, \dot{\omega})$ is an element of $\mathcal{V}$, it must satisfy $L(X, \dot{\omega})=0$, that is, it must be a Jacobi field.

Lemma 8.1. If $\Sigma$ has genus at least two, then $\mathcal{V}=0$ and $\mathcal{P}_{s}$ is a smooth submanifold of $\mathcal{A}(\Sigma, M)$. If $\Sigma$ has genus zero or one, then each $\mathcal{V}_{i}=0$ and each $\mathcal{P}_{s, i}$ is a smooth submanifold of $\mathcal{A}_{i}(\Sigma, M)$.

To prove this, we need to modify the arguments presented in $₫ 3$ and $₫ 6$ to handle the case in which $f$ may have simple branch points.

We first note that Lemma 6.1 applies directly, and shows that the only elements $(X, \dot{\omega})$ of $\mathcal{V}_{i}$ (when the genus of $\Sigma$ is $\leq 1$ ) or $\mathcal{V}$ (when the genus of $\Sigma$ is $\geq 2$ ) are elements of the space $\mathcal{J}\left(\left(f^{*} T M\right)^{\top}\right)$ of tangential Jacobi fields.

Recall from Remark 5.1 that this space consists of pairs $(X, \dot{\omega})$, where $X$ is the real part of a section $Z$ of $\mathbf{L}$ and $\dot{\omega} \in T_{\omega} \mathcal{T}$ corresponds to a holomorphic quadratic differential $h d z^{2}$ such that $\left(Z, h d z^{2}\right)$ satisfies (68). In the case where $\Sigma=S^{2}$, we let

$$
\mathcal{J}_{0}\left(\left(f^{*} T M\right)^{\top}\right)=\left\{(X, \dot{\omega}) \in \mathcal{J}\left(\left(f^{*} T M\right)^{\top}\right): X(q)=X(r)=X(s)=0\right\},
$$

where $q, r$ and $s$ are the distinguished points of $S^{2}$ chosen before. In the case where $\Sigma=T^{2}$, we let

$$
\mathcal{J}_{0}\left(\left(f^{*} T M\right)^{\top}\right)=\left\{(X, \dot{\omega}) \in \mathcal{J}\left(\left(f^{*} T M\right)^{\top}\right): X(q)=0\right\} .
$$

When $\Sigma$ has genus at least two, we simply let $\mathcal{J}_{0}\left(\left(f^{*} T M\right)^{\top}\right)=\mathcal{J}\left(\left(f^{*} T M\right)^{\top}\right)$. (Note that since the vector fields $X$ are tangential to $\Sigma$, requiring that they be tangential to the codimension two submanifolds $Q_{i}, R_{i}, S_{i}$ or $N_{i}$ is equivalent to requiring that they vanish at the corresponding points.) Finally, we let $\mathcal{J}_{0}(\mathbf{L})$ denote the corresponding space of sections of $\mathbf{L}$, in the various cases. 
We claim that under our assumption that $f$ has only simple branch points, it follows from Lemma 7.1 that the derivative $D_{2} F$ with respect to the metric on the ambient space $M$ maps onto $\mathcal{J}_{0}\left(\left(f^{*} T M\right)^{\top}\right)$. Indeed, it follows from (67) in $\$ 6$ that $D_{2} F_{1}$ is given by

$$
\left\langle\left\langle D_{2} F_{1}(f, \omega, g)\left(\dot{g}_{j}\right), \dot{\omega}\right\rangle\right\rangle=-\int_{\Sigma} \frac{2}{\sigma^{2}}\left\langle\frac{\bar{k}}{\lambda^{2}} \frac{\partial f}{\partial z}, \frac{h_{j}}{\lambda^{2}} \frac{\partial f}{\partial \bar{z}}\right\rangle d A,
$$

when $\dot{g}_{j}$ is the metric deformation corresponding to the quadratic differential $h_{j} d z^{2}$, and $\dot{\omega}$ is the element of the tangent space $T_{\omega} \mathcal{T}$ to the Teichmüller space of $\Sigma$ corresponding to the holomorphic quadratic differential $k d z^{2}$. We want to apply this formula to the case where $h_{j} d z^{2}$ is a quadratic differential corresponding to one of the deformations $\dot{g}_{j}$ in the metric constructed in the proof of Lemma 7.1.

We observe, however, that there is some freedom in constructing the quadratic differential $h_{j} d z^{2}$; recall that it was constructed from a meromorphic differential $h_{j}^{\prime}$ with a simple pole at $p_{j}$ and no other poles. To $h_{j}^{\prime}$ we could have added an arbitrary holomorphic quadratic differential. Indeed, it follows from Lemma 7.1 that we can add such a holomorphic quadratic differential to $h_{j}^{\prime}$ which makes (83) vanish for every choice of holomorphic quadratic differential $k d z^{2}$. It is then the case that

$$
\left\langle\left\langle D_{2} F(f, \omega, g)\left(\dot{g}_{j}\right),(X, \dot{\omega})\right\rangle\right\rangle=\left\langle\left\langle D_{2} F_{0}(f, \omega, g)\left(\dot{g}_{j}\right), X\right\rangle\right\rangle=\operatorname{Re}\left(B\left(h_{j}, Z\right)\right),
$$

when $(X, \dot{\omega})$ is an element of $\mathcal{J}_{0}\left(\left(f^{*} T M\right)^{\top}\right)$ which corresponds to the element $Z \in$ $\mathcal{J}_{0}(\mathbf{L})$. Hence the family of variations $\left\{\dot{g}_{j}\right\}$ in the metric that was constructed in Lemma 7.1 perturbs away all elements of $\mathcal{J}_{0}\left(\left(f^{*} T M\right)^{\top}\right)$, and hence $\mathcal{V}_{i}=0$ or $\mathcal{V}=0$, depending on the genus of $\Sigma$.

Thus we see that the restriction of $F$ to $\mathcal{P}_{s}$ when $\Sigma$ has genus at least two, or to each $\mathcal{P}_{s, i}$ when $\Sigma$ has genus zero or one, is transverse to the zero section. The implicit function theorem shows once again that $\mathcal{P}_{s}$ or each of the $\mathcal{P}_{s, i}$ 's is a submanifold of $\mathcal{A}(\Sigma, M)$ or $\mathcal{A}_{i}(\Sigma, M)$, finishing the proof of Lemma 8.1.

Lemma 8.2. If $\Sigma$ has genus at least two, then the projection $\pi: \mathcal{P}_{s} \rightarrow \operatorname{Met}(M)$ is a Fredholm map of Fredholm index zero. If $\Sigma$ has genus zero or one, then the projection $\pi: \mathcal{P}_{s, i} \rightarrow \operatorname{Met}(M)$ is a Fredholm map of Fredholm index zero, for each $i$.

To prove this, we start by noting that when $\Sigma$ has genus at least two, the tangent space to $\mathcal{P}_{s}$ at $(f, \omega, g)$ is

$$
\begin{aligned}
T_{(f, \omega, g)} \mathcal{P}_{s}=\left\{(X, \dot{\omega}, h) \in T_{f} \operatorname{Map}(\Sigma, M) \oplus T_{\omega} \mathcal{T} \oplus T_{g} \operatorname{Met}(M):\right. \\
\left.L(X, \dot{\omega})+\pi_{V} \circ\left(D_{2} F\right)_{(f, \omega, g)}(h)=0\right\},
\end{aligned}
$$

a formula just like that used in 86 . When $\Sigma$ has genus zero or one, the tangent space to $\mathcal{P}_{s, i}$ is the same except for the additional stipulation that $X$ vanish at the distinguished points.

From here the proof proceeds in exactly the same way as the proofs in 93 and $\sqrt[6]{6}$ with one simplification. Since we have destroyed the $G$-symmetry, both projections

$$
\pi: \mathcal{P}_{s} \rightarrow \operatorname{Met}(M) \quad \text { and } \pi: \mathcal{P}_{s, i} \rightarrow \operatorname{Met}(M)
$$

are Fredholm maps of Fredholm index zero.

To finish the proof of Proposition 8.1, we note that if $(f, \omega, g)$ is a critical point for the energy $E$ and $f$ has a nontrivial divisor of simple branch points, then Lemma 7.1 
provides a variation $h$ in the metric on $M$ such that $\pi_{V} \circ\left(D_{2} F\right)_{(f, \omega, g)}(h)$ does not lie in the image of the Jacobi operator $L$. It follows that the metric variation $h$ cannot lie in the image of $d \pi$ at $(f, \omega, g)$ and perturbation of the metric in the direction of $h$ will take us out of the submanifold $\mathcal{P}_{s}$. In particular, if $g$ admits parametrized minimal surfaces with a nontrivial divisor of simple branch points, then $g$ cannot be a regular value. Therefore such minimal surfaces cannot occur for generic choice of metric.

\section{StratificAtion ACCORDING TO BRANCH TYPE}

In this section, as preparation for considering conformal harmonic maps which may have branch points of order two or greater, we construct a submanifold of finite codimension in

$$
\operatorname{Map}(\Sigma, M) \times \mathcal{T} \times \operatorname{Met}(\Sigma)
$$

which consists of conformal maps of a given "branch type", as described below.

Throughout this section, we take $\operatorname{Map}(\Sigma, M)$ to be the completion of the smooth maps with respect to the $L_{k}^{2}$ topology and $\operatorname{Met}(M)$ the completion of the smooth metrics with respect to the $L_{k-1}^{2}$ topology, where $k$ is chosen to be sufficiently large (depending on the order of the branch points considered). We take the diffeomorphisms of $\Sigma$ to be $C^{\infty}$.

By a branch type for total branching order $\nu$, we mean a sequence of positive integers $\left(\nu_{1}, \ldots, \nu_{n}\right)$, such that

$$
\nu_{1} \geq \nu_{2} \geq \cdots \geq \nu_{n} \quad \text { and } \quad \sum_{i=1}^{n} \nu_{i}=\nu .
$$

We sometimes denote the total branching order (which will also be called the degree) of $\Lambda$ by $|\Lambda|$ and note that there are only finitely many branch types $\Lambda$ such that $|\Lambda|=\nu$. We define a partial order on branch types by demanding that if $\Lambda=$ $\left(\nu_{1}, \ldots, \nu_{n}\right)$ and $\Lambda^{\prime}=\left(\nu_{1}^{\prime}, \ldots, \nu_{n^{\prime}}^{\prime}\right)$, then

$$
\Lambda^{\prime} \leq \Lambda \quad \Leftrightarrow \quad n \leq n^{\prime} \quad \text { and } \quad \nu_{i} \leq \nu_{i}^{\prime} \quad \text { for } 1 \leq i \leq n .
$$

We write $\Lambda^{\prime}<\Lambda$ if $\Lambda^{\prime} \leq \Lambda$ and $\Lambda^{\prime} \neq \Lambda$. There is a unique branch type $\emptyset$ of total branching order 0 , and $\Lambda \leq \emptyset$ for every branch type $\Lambda$. We say that a divisor $D$ on $\Sigma$ has branch type $\Lambda=\left(\nu_{1}, \ldots, \nu_{n}\right)$ if there is an ordered collection of distinct points $\left(p_{1}, \ldots, p_{n}\right)$ such that $D=\sum \nu_{i} p_{i}$, and that a harmonic map $f: \Sigma \rightarrow M$ has branch type $\Lambda$ if its divisor of branch points $D(f)$ has branch type $\Lambda$.

We now describe submanifolds of $\operatorname{Map}(\Sigma, M)$, of finite codimension, corresponding to each branch type. In the case $\Lambda=\emptyset$, we set

$$
\operatorname{Map}_{\emptyset}(\Sigma, M)=\{f \in \operatorname{Map}(\Sigma, M): f \text { is an immersion }\} .
$$

Suppose that $\Lambda \neq \emptyset$. Given a divisor $D=\sum \nu_{i} p_{i}$ of branch type $\Lambda$, we let

$$
\begin{aligned}
& \operatorname{Map}_{\Lambda, D}(\Sigma, M)=\{f \in \operatorname{Map}(\Sigma, M): f \text { is an immersion except at } \\
& \text { each point } p_{i} \text { where the } \nu_{i} \text {-jet of } f \text { vanishes } \\
& \text { but the } \left.\left(\nu_{i}+1\right) \text {-jet does not, for } 1 \leq i \leq n\right\} .
\end{aligned}
$$

(By saying that the $\nu$-jet of $f$ vanishes at $p$, we simply mean that all covariant derivatives of $f$ of order between one and $\nu$ vanish at $p$.) Using the Sobolev imbedding theorem and the smoothness of the $\nu_{i}$-jet evaluation map described in [5], 
Theorem 10.4, for manifolds of $C^{k}$ maps, one checks that this is a $C^{k-\nu_{0}-2}$ submanifold of finite codimension

$$
\sum_{i=1}^{n} \sum_{j=1}^{\lambda_{i}}(j+1) \operatorname{dim} M
$$

in $\operatorname{Map}(\Sigma, M)$, where $\nu_{0}$ is the maximum of $\nu_{1}, \ldots, \nu_{n}$. In the special case $\Lambda=$ $(1, \ldots, 1)$ and $D=p_{1}+\cdots+p_{n}$, the tangent space is given by

$$
T_{f}\left(\operatorname{Map}_{\Lambda, D}(\Sigma, M)\right)=\left\{X \in T_{f}(\operatorname{Map}(\Sigma, M)): \nabla X\left(p_{i}\right)=0 \text {, for } 1 \leq i \leq n\right\} .
$$

Note that $X\left(p_{i}\right)$ itself is not necessarily zero. In the general case,

$$
\begin{aligned}
& T_{f}\left(\operatorname{Map}_{\Lambda, D}(\Sigma, M)\right) \\
& \quad=\left\{X \in T_{f}(\operatorname{Map}(\Sigma, M)): \nabla^{j} X\left(p_{i}\right)=0, \text { for } 1 \leq j \leq \lambda_{i} \text { and } 1 \leq i \leq n\right\},
\end{aligned}
$$

where $\nabla^{j} X$ denotes the $j$-th covariant differential of $X$. If $f \in\left(\operatorname{Map}_{\Lambda, D}(\Sigma, M)\right)$, we call $D$ the divisor of $f$ and write $D(f)=D$.

However, the space that really interests us is the space of maps of branch type $\Lambda$, in which the points are unspecified,

$$
\begin{aligned}
\operatorname{Map}_{\Lambda}(\Sigma, M)=\left\{f \in \operatorname{Map}(\Sigma, M): f \in \operatorname{Map}_{\Lambda, D}(\Sigma, M)\right. & \\
& \text { for some divisor } D \text { of branch type } \Lambda\} .
\end{aligned}
$$

If we let $\mathcal{C}_{n}$ denote the configuration space of ordered $n$-tuples of distinct points in $\Sigma$, we have a fibration

$$
\pi: \operatorname{Map}_{\Lambda}(\Sigma, M) \rightarrow \mathcal{C}_{n}
$$

in which the fibers are the spaces $\operatorname{Map}_{\Lambda, D}(\Sigma, M)$ considered before, each of which is mapped to $\left(p_{1}, \ldots, p_{n}\right)$ when $D=\sum \nu_{i} p_{i}$.

We can determine the tangent space to $\operatorname{Map}_{\Lambda}(\Sigma, M)$ at a point $f$ in the case where $\Lambda=(1,1, \ldots, 1)$ as follows: Suppose that $D(f)=p_{1}+\cdots+p_{n}$, so that $d f\left(p_{i}\right)=0$ for $1 \leq i \leq n$. About a given point $p_{i}$ we choose local coordinates $\left(x_{1}, x_{2}\right)$ such that $x_{1}\left(p_{i}\right)=x_{2}\left(p_{i}\right)=0$. Moreover, for $t \in(-\epsilon, \epsilon)$, suppose that

$$
\begin{gathered}
t \mapsto f_{t} \quad \text { is a variation in } \operatorname{Map}_{\Lambda}(\Sigma, M) \text { with } f_{0}=f \\
\text { and } \quad t \mapsto \gamma(t) \quad \text { is a curve in } M \text { with } \gamma(0)=p
\end{gathered}
$$

such that $d f_{t}(\gamma(t))=0$. If $x_{a}(t)=x_{a} \circ \gamma(t)$, for $1 \leq a \leq 2$, then

$$
\frac{\partial^{2} f}{\partial x_{a} \partial t}(p)+\sum_{b=1}^{2} \frac{\partial^{2} f}{\partial x_{a} \partial x_{b}}(p) x_{b}^{\prime}(t)=0 .
$$

In more invariant notation, we can write this as

$$
\nabla X\left(p_{i}\right)+d^{2} f\left(p_{k}\right)\left(v_{i}, \cdot\right)=0, \quad \text { where } \quad X=\frac{\partial f_{t}}{\partial t} \quad \text { and } \quad v_{i}=\gamma^{\prime}(0) .
$$

This condition could also be obtained from the explicit derivatives of the one-jet evaluation map as described in Abraham and Robbin [5, page 27. 
Thus to allow for motion of each point $p_{i}$ in the direction of some vector $v_{i} \in T_{p_{i}} \Sigma$ requires that we modify (86) to

$$
\begin{aligned}
& T_{f}\left(\operatorname{Map}_{\Lambda}(\Sigma, M)\right)=\left\{X \in T_{f}(\operatorname{Map}(\Sigma, M))\right. \\
& \left.\nabla X\left(p_{i}\right)=-d^{2} f\left(p_{i}\right)\left(v_{i}, \cdot\right), \text { for some } v_{i} \in T_{p_{i}} \Sigma, \text { for } 1 \leq i \leq n\right\} .
\end{aligned}
$$

Note that $\nabla X\left(p_{i}\right)$ and $d^{2} f\left(p_{i}\right)\left(v_{i}, \cdot\right)$ are both linear maps from $T_{p_{i}} \Sigma$ to $T_{f\left(p_{i}\right)} M$. In the general case, the tangent space is

$$
\begin{aligned}
& \left.T_{f} \operatorname{Map}_{\Lambda}(\Sigma, M)\right) \\
& =\left\{X \in T_{f}(\operatorname{Map}(\Sigma, M)): \nabla^{j} X\left(p_{i}\right)=0, \text { for } 1 \leq j \leq \lambda_{i-1},\right. \text { and } \\
& \left.\quad \nabla^{\nu_{i}} X\left(p_{i}\right)=-\left(d^{\nu_{i+1}} f\right)\left(p_{i}\right)\left(v_{i}, \cdot, \ldots, \cdot\right), \text { for } v_{i} \in T_{p_{i}} \Sigma, 1 \leq i \leq n\right\} .
\end{aligned}
$$

Once again, it is relatively straightforward (using smoothness of the $\nu_{i}$-jet evaluation maps) to show that $\operatorname{Map}_{\Lambda}(\Sigma, M)$ is a smooth manifold (of finite codimension) with this tangent space.

Unfortunately, the space $\operatorname{Map}_{\Lambda}(\Sigma, M)$ contains maps which cannot be conformal with respect to any choice of metrics on $\Sigma$ and $M$. For our application to conformal harmonic maps, we need to consider an open subset of $\operatorname{Map}_{\Lambda}(\Sigma, M)$ defined by a nondegeneracy condition at the points with nonzero coefficient in the divisor. Let $p$ be one of these and suppose that its coefficient is $\nu$. Then

$$
d^{\nu+1} f: T_{p} \Sigma \times \cdots \times T_{p} \Sigma \longrightarrow T_{f(p)} M
$$

is a polynomial approximation to $f$ near $p$, which we can write as

$$
u=\left(u_{1}, u_{2}, \ldots, u_{n}\right)=P\left(x_{1}, x_{2}\right)
$$

in terms of local coordinates, with $P$ a vector-valued homogeneous polynomial. Indeed, we can choose Riemannian metrics on $\Sigma$ and $M$ and regard $\left(x_{1}, x_{2}\right)$ as normal coordinates on a ball $B_{r}(p)$ of radius $r$ centered at $p \in \Sigma$ and $\left(u_{1}, \ldots, u_{n}\right)$ as normal coordinates centered at $f(p) \in M$. We can then take $P$ to be an $\mathbb{R}^{n_{-}}$ valued homogeneous polynomial of degree $\nu+1$ in the variables $\left(x_{1}, x_{2}\right)$. Consider the following condition on $d^{\nu+1} f$ :

$$
\left|\frac{\partial P}{\partial x_{1}} \wedge \frac{\partial P}{\partial x_{2}}\right| \neq 0 \quad \text { unless } \quad x_{1}=x_{2}=0
$$

a condition which is invariant under change of metrics and coordinates. We let

$$
\begin{aligned}
& \operatorname{Map}_{\Lambda}^{\prime}(\Sigma, M)=\left\{f \in \operatorname{Map}_{\Lambda}(\Sigma, M):\right. \\
& f \text { satisfies condition (87) at every point } p \text { such that } \\
& \text { the } \nu \text {-jet of } f \text { at } p \text { vanishes for some } \nu \geq 1\} .
\end{aligned}
$$

Then $\operatorname{Map}_{\Lambda}^{\prime}(\Sigma, M)$ is an open subset of $\operatorname{Map}_{\Lambda}(\Sigma, M)$ which contains all conformal harmonic maps $f: \Sigma \rightarrow M$ for whatever metric is chosen on $M$ and whatever conformal structure is chosen on $\Sigma$.

Let $p$ be a potential branch point of order $\nu$ for an element $f \in \operatorname{Map}_{\Lambda}^{\prime}(\Sigma, M)$, and let $\left(x_{1}, x_{2}\right)$ be normal coordinates as described above. Denote the metric induced by $f$ on a neighborhood of $p$ in $\Sigma$ by $\eta=\sum \eta_{i j} d x_{1} d x_{j}$. Then condition (87) implies that there exist positive constants $c_{1}$ and $c_{2}$ such that

$$
c_{1} r^{2 \nu}\left(d x_{1}^{2}+d x_{2}^{2}\right) \prec \sum \eta_{i j} d x_{1} d x_{j} \prec c_{2} r^{2 \nu}\left(d x_{1}^{2}+d x_{2}^{2}\right),
$$


where $h_{1} \prec h_{2}$ means that $h_{2}-h_{1}$ is positive definite and $r=\sqrt{x_{1}^{2}+x_{2}^{2}}$.

Lemma 9.1. Suppose that $f \in \operatorname{Map}_{\Lambda}^{\prime}(\Sigma, M)$. Then any Riemannian metric on $M$ induces a conformal structure on $\Sigma$ with respect to which $f$ is conformal.

To prove this, note first that $f$ clearly induces a conformal structure on the surface $\Sigma-\left\{p_{1}, \ldots, p_{n}\right\}$, where $D(f)=\nu_{1} p_{1}+\cdots+\nu_{n} p_{n}$. We claim that the nondegeneracy condition in the definition of $\operatorname{Map}_{\Lambda}^{\prime}(\Sigma, M)$ implies that the conformal structure extends to all of $\Sigma$.

Let $p$ be one of the points with nonzero coefficient in the divisor, and use polar coordinates $(r, \theta)$ related to normal coordinates $\left(x_{1}, x_{2}\right)$ centered at $p$ by $x_{1}=$ $r \cos \theta, x_{2}=r \sin \theta$. Then we can rewrite (88) as

$$
c_{1} r^{2 \nu}\left(d r^{2}+r^{2} d \theta^{2}\right) \prec \eta \prec c_{2} r^{2 \nu}\left(d r^{2}+r^{2} d \theta^{2}\right),
$$

and making the substitution $r=e^{-u}$ yields

$$
c_{1} e^{-(2 \nu+2) u}\left(d u^{2}+d \theta^{2}\right) \prec \eta \prec c_{2} e^{-(2 \nu+2) u}\left(d u^{2}+d \theta^{2}\right) .
$$

Thus the metric on the deleted disk is quasiconformal to the standard metric $e^{-(2 \nu+2) u}\left(d u^{2}+d \theta^{2}\right)$ on the punctured disk, a metric which contains arbitrarily long annuli, that is, annuli conformal to

$$
A=\left\{z \in \mathbb{C}: r_{1}<|z|<r_{2}\right\}, \quad \text { for which the invariant } \mathfrak{m}(A)=\frac{1}{2 \pi} \log \left(\frac{r_{2}}{r_{1}}\right)
$$

is arbitrarily large. $(\mathfrak{m}(A)$ classifies conformal structures on annuli.) It follows (for example from Lemma 2, page 51 of [1], or from the theory presented in $\S 1$ of Chapter I in 22]) that the metric $\eta$ on $B_{r}(p)-\{p\}$ also contains arbitrarily long conformal cylinders, and hence that in the conformal structure for the metric $\eta$, $B_{r}(p)-\{p\}$ is a punctured disk. This implies that the conformal structure can be extended to $p$.

Since this conclusion holds for all points with nonzero coefficient in the divisor, the conformal structure can be extended to $\Sigma$ and the lemma is proven.

We will need to consider the space of maps from $\Sigma$ to $M$ which are conformal with respect to some choice of metrics on domain and range, but not necessarily harmonic, maps which are allowed to have isolated singularities similar to branch points. To define the space of such maps, we first suppose that $\omega$ is a conformal structure on the oriented closed surface $\Sigma$ and that $g=\langle\cdot, \cdot\rangle$ is a Riemannian metric on the ambient manifold $M$. We say that $f \in \operatorname{Map}_{\Lambda}^{\prime}(\Sigma, M)$ is conformal with respect to $\omega$ and $g$ if whenever $z=x+i y$ is a parameter on $\Sigma$ which is conformal for $\omega$,

$$
\left\langle\frac{\partial f}{\partial z}, \frac{\partial f}{\partial z}\right\rangle \equiv 0
$$

If $\Lambda=\left(\nu_{1}, \ldots, \nu_{n}\right)$, and $\partial f / \partial z$ vanishes to order $\nu_{i}$ at $p_{i}$, for $1 \leq i \leq n$, we say that $f$ has divisor

$$
D(f)=\nu_{1} p_{1}+\nu_{2} p_{2}+\cdots+\nu_{n} p_{n}
$$

and that $f$ has a branch point of order $\nu_{i}$ at each $p_{i}$.

Lemma 9.2. If $f \in \operatorname{Map}_{\Lambda}^{\prime}(\Sigma, M)$ is conformal and has a branch point of order $\nu$ at $p$, there is a conformal coordinate $z$ centered at $p$ such that

$$
\frac{\partial f}{\partial z}=z^{\nu} g, \quad \text { with } \quad g(p) \neq 0, \quad\langle g, g\rangle=0 .
$$


To prove this lemma, we note that differentiation of (89) shows that if $\partial f / \partial z$ vanishes at a point $p$, then

$$
\left\langle\frac{\partial^{2} f}{\partial z^{2}}, \frac{\partial^{2} f}{\partial z^{2}}\right\rangle(p)=0, \quad\left\langle\frac{\partial^{2} f}{\partial \bar{z} \partial z}, \frac{\partial^{2} f}{\partial \bar{z} \partial z}\right\rangle(p)=0,
$$

where we have used the notation for partial derivatives instead of covariant derivatives since the first order derivatives vanish at $p$, and hence the second order covariant derivatives at $p$ are independent of connection. The second of these conditions implies that

$$
\left\langle\frac{\partial^{2} f}{\partial x^{2}}+\frac{\partial^{2} f}{\partial y^{2}}, \frac{\partial^{2} f}{\partial x^{2}}+\frac{\partial^{2} f}{\partial y^{2}}\right\rangle(p)=0
$$

and since the metric $g$ is positive definite,

$$
\frac{\partial^{2} f}{\partial x^{2}}(p)=-\frac{\partial^{2} f}{\partial y^{2}}(p)
$$

Thus the space spanned by the second-order partial derivatives of $f$ is two-dimensional, spanned by

$$
\frac{\partial^{2} f}{\partial x^{2}}(p) \quad \text { and } \quad \frac{\partial^{2} f}{\partial x \partial y}(p)
$$

We can also express this as follows: if $\partial f / \partial z$ vanishes to order one at $p$, then

$$
\frac{\partial^{2} f}{\partial \bar{z} \partial z}(p)=0, \quad\left\langle\frac{\partial^{2} f}{\partial z^{2}}, \frac{\partial^{2} f}{\partial z^{2}}\right\rangle(p)=0 .
$$

If $\partial f / \partial z$ vanishes to order two at $p$, then all second order partial derivatives must vanish, and by an argument similar to that of the previous paragraph, we see that

$$
\frac{\partial^{3} f}{\partial \bar{z}^{2} \partial z}(p)=\frac{\partial^{3} f}{\partial \bar{z} \partial z^{2}}(p)=0, \quad\left\langle\frac{\partial^{3} f}{\partial z^{3}}, \frac{\partial^{3} f}{\partial z^{3}}\right\rangle(p)=0 .
$$

Similarly, if $\partial f / \partial z$ vanishes to order $\nu$ at $p$, where $\nu \geq 3$, then all partial derivatives of $f$ of order $\leq \nu+1$ vanish at $p$ except for

$$
\frac{\partial^{\nu+1} f}{\partial z^{\nu+1}} \quad \text { and } \quad \frac{\partial^{\nu+1} f}{\partial \bar{z}^{\nu+1}}, \quad \text { and } \quad\left\langle\frac{\partial^{\nu+1} f}{\partial z^{\nu+1}}, \frac{\partial^{\nu+1} f}{\partial z^{\nu+1}}\right\rangle(p)=0 .
$$

If $\partial f / \partial z$ vanishes to order $\nu$ at $p$ and $z(p)=0$, Taylor's theorem and the above conditions on derivatives imply that we can perform a factorization

$$
\frac{\partial f}{\partial z}=z^{\nu} g, \quad \text { where } \quad g: \Sigma \rightarrow f^{*} T M \otimes \mathbb{C}
$$

is a smooth section satisfying the conditions that $g(p) \neq 0$ and $\langle g, g\rangle=0$, finishing the proof of Lemma 9.2.

The importance of this lemma is that it allows us to carry out the construction of the line bundle $\mathbf{L}$ described in $\oint 2$ for maps $f$ which lie in $\operatorname{Map}_{\Lambda}^{\prime}(\Sigma, M)$. When we give $\Sigma$ the induced conformal structure, we can describe $\mathbf{L}$ as the line bundle summand lying within the complexified tangent bundle $f^{*} T M \otimes \mathbb{C}$ such that for each choice of conformal parameter $z, \partial f / \partial z$ is a section of $\mathbf{L}$. Note that all sections of $\mathbf{L}$ are isotropic with respect to the complex bilinear extension of the Riemannian metric on $M$. The line bundle $\mathbf{L}$ inherits a metric and connection from $f^{*} T M \otimes \mathbb{C}$, as well as a Koszul-Malgrange holomorphic structure. Of course, $\mathbf{L}$ will not be a holomorphic subbundle of $f^{*} T M \otimes \mathbb{C}$ unless $f$ is not only conformal, but also harmonic. 
However, we can define the space of holomorphic sections $\mathcal{O}(\mathbf{L})$ of $\mathbf{L}$, as well as the larger space $\mathcal{J}(\mathbf{L})$ of sections $Z$ described in (60), sections for which the equation (58) has a solution for suitable choice of holomorphic quadratic differential. In accordance with (61), we can also define the isomorphic space $\mathcal{J}\left(\left(f^{*} T M\right)^{\top}\right)$ of pairs $(X, \dot{\omega})$, where $X$ is the real part of $Z$ and $\dot{\omega}$ is an element of the tangent space to the Teichmüller space $\mathcal{T}$. Note that these spaces can be defined even at points which are not critical for $E$, and can be regarded as "potential" tangential Jacobi fields. It is readily verified that Lemma 5.1 still holds for conformal maps which are not harmonic, so the isomorphic spaces $\mathcal{J}(\mathbf{L})$ and $\mathcal{J}\left(\left(f^{*} T M\right)^{\top}\right)$ have dimension which depends only on the total branching order $\nu=\sum \nu_{i}$ of a conformal map with divisor given by (90).

We can now consider the restriction of the energy function,

$$
E: \widetilde{\mathcal{B}}_{\Lambda}(\Sigma, M) \longrightarrow \mathbb{R}, \quad \text { where } \widetilde{\mathcal{B}}_{\Lambda}(\Sigma, M)=\operatorname{Map}_{\Lambda}^{\prime}(\Sigma, M) \times \operatorname{Met}_{0}(\Sigma) \times \operatorname{Met}(M) .
$$

The first variation formula, restricted to variations with compact support avoiding the branch points, shows that critical points of this restriction will be harmonic except possibly at the branch points, but by continuity they will have to be harmonic at the branch points as well. Thus the Hopf differential $\Omega_{f}$ described in 92 will be holomorphic and a critical point $(f, \eta, g)$ will be conformal with respect to the conformal structures defined by $\eta$ and $g$. Of course, $E$ is invariant under the action of the group $\operatorname{Diff}_{0}(\Sigma)$ of diffeomorphisms isotopic to the identity, so it descends to the quotient

$$
\mathcal{B}_{\Lambda}(\Sigma, M)=\frac{\widetilde{\mathcal{B}}_{\Lambda}(\Sigma, M)}{\operatorname{Diff}_{0}(\Sigma)} \cong \operatorname{Map}_{\Lambda}^{\prime}(\Sigma, M) \times \mathcal{T} \times \operatorname{Met}(M),
$$

and we can regard the restricted energy as a map

$$
E: \mathcal{B}_{\Lambda}(\Sigma, M) \longrightarrow \mathbb{R}
$$

We can summarize the preceding discussion as follows.

Lemma 9.3. Over the space $\mathcal{B}_{\Lambda}(\Sigma, M)$, we have a smoothly varying family of line bundles $\mathbf{L} \subset f^{*} T M \otimes \mathbb{C}$, isotropic with respect to $\langle\cdot, \cdot\rangle$. Moreover, we have a family of spaces $\mathcal{J}(\mathbf{L})$, solutions to (58) for appropriate choices of holomorphic quadratic differentials, which varies smoothly with $(f, \omega, g) \in \mathcal{B}_{\Lambda}(\Sigma, M)$.

\section{Proof of the Main Theorem}

To finish the proof of the Main Theorem, it suffices to show that if $M$ is a compact connected manifold of dimension at least three, then for a generic choice of Riemannian metric on $M$, there are no prime minimal surfaces with branch points that have branching order two or larger.

The argument is similar to that presented in $₫ 8$, except that we must now deal with the added complication that there are additional Jacobi fields, not forced by the group action, that are not covered by the variations in the metric on $M$ described in Lemma 7.1. We will use the stratification described in $\$ 9$ and will 
restrict our attention to one branch type at a time. Thus we let

$$
\begin{aligned}
& \mathcal{P}_{\Lambda}=\{(f, \omega, g) \in \operatorname{Map}(\Sigma, M) \times \mathcal{T} \times \operatorname{Met}(M): \\
& f \text { is a prime conformal } \omega \text {-harmonic map } \\
& \text { for } g \text { which has branch type } \Lambda\} .
\end{aligned}
$$

The Main Theorem will then be a consequence of the following lemma and the Sard-Smale Theorem.

Lemma 10.1. When $\Lambda \neq \emptyset$, all elements of $\mathcal{P}_{\Lambda}$ lie on a countable collection of submanifolds $\mathcal{Q}_{\Lambda, i}$ each of which has Fredholm projection to $\operatorname{Met}(M)$. Moreover, at each point of $\mathcal{Q}_{\Lambda, i}$ with $\Lambda \neq \emptyset$, the cokernel of $d \pi$ has dimension at least two.

We remark that when applied to the case of simple branch points, for which $\Lambda=$ $(1, \ldots, 1)$, the argument presented below leads to an alternate proof of Proposition 8.1 .

Our strategy is to show that there is a countable cover $\left\{\mathcal{W}_{i}: i \in I\right\}$ of $\mathcal{P}_{\Lambda}$ by open subsets of

$$
\mathcal{A}(\Sigma, M)=\operatorname{Map}(\Sigma, M) \times \mathcal{T} \times \operatorname{Met}(M)
$$

such that

$$
\mathcal{W}_{i} \cap \mathcal{P}_{\Lambda} \subset \mathcal{Q}_{\Lambda, i}
$$

where $\mathcal{Q}_{\Lambda, i}$ has Fredholm projection to $\operatorname{Met}(M)$, the cokernel having dimension at least two. If $\Sigma$ has genus zero or one, we modify the strategy a little by replacing $\mathcal{A}(\Sigma, M)$ by a submanifold of codimension six or two respectively, in order to break the symmetry, just as we did in 8,8 .

In the case of the sphere, we break the $\operatorname{PSL}(2, \mathbb{C})$ symmetry by choosing the elements of the cover $\left\{\mathcal{W}_{i}: i \in I\right\}$ so that

$$
\mathcal{W}_{i} \subset\left\{(f, \omega, g) \in \mathcal{A}\left(S^{2}, M\right): f \in \mathcal{U}\left(Q_{i}, R_{i}, S_{i}\right)\right\},
$$

where $i \mapsto \mathcal{U}\left(Q_{i}, R_{i}, S_{i}\right)$ is a sequence of sets as described in the proof of Proposition 8.1. In the case of the torus, we break the $S^{1} \times S^{1}$ symmetry by choosing $\mathcal{W}_{i}$ so that

$$
\mathcal{W}_{i} \subset\left\{(f, \omega, g) \in \mathcal{A}\left(T^{2}, M\right): f \in \mathcal{U}\left(N_{i}\right)\right\},
$$

where $i \mapsto \mathcal{U}\left(N_{i}\right)$ is a sequence of sets as described later in the proof of Proposition 8.1. We then define $\mathcal{F}_{i}\left(S^{2}, M\right)$ and $\mathcal{F}_{i}\left(T^{2}, M\right)$ by (80) and (81), and set

$$
\begin{aligned}
& \mathcal{A}_{i}(\Sigma, M)=\left\{(f, \omega, g) \in \mathcal{A}(\Sigma, M) \cap \mathcal{W}_{i}: f \in \mathcal{F}_{i}\left(T^{2}, M\right)\right\}, \\
& \mathcal{P}_{\Lambda, i}=\mathcal{P}_{\Lambda} \cap \mathcal{A}_{i}(\Sigma, M) .
\end{aligned}
$$

If $\Sigma$ has genus at least two, we keep a uniform notation by setting

$$
\mathcal{A}_{i}(\Sigma, M)=\mathcal{A}(\Sigma, M) \cap \mathcal{W}_{i}, \quad \mathcal{P}_{\Lambda, i}=\mathcal{P}_{\Lambda} \cap \mathcal{W}_{i} .
$$

It is at this point that the submanifolds $\mathcal{B}_{\Lambda}(\Sigma, M)$ introduced in 99 play a crucial role. Note that the space $\mathcal{P}_{\Lambda}$ is contained in the submanifold $\mathcal{B}_{\Lambda}(\Sigma, M)$ of $\mathcal{A}(\Sigma, M)$ and $\mathcal{P}_{\Lambda, i}$ is contained in the submanifold

$$
\mathcal{B}_{\Lambda, i}(\Sigma, M)=\mathcal{B}_{\Lambda}(\Sigma, M) \cap \mathcal{A}_{i}(\Sigma, M)
$$

of $\mathcal{A}_{i}(\Sigma, M)$. Suppose that $E_{\mathcal{B}}$ is the model Banach space for $\mathcal{B}_{\Lambda, i}(\Sigma, M)$ and $E_{\mathcal{A}}$ is the model space for $\mathcal{A}_{i}(\Sigma, M)$, with $E_{\mathcal{A}}=E_{\mathcal{B}} \oplus F$, where $F$ is the finite-dimensional 
complement. We can then choose the cover $\left\{\mathcal{W}_{i}: i \in I\right\}$ so that each $\mathcal{A}_{i}(\Sigma, M)$ is the domain of a smooth chart

$$
\phi: \mathcal{A}_{i}(\Sigma, M) \rightarrow E_{\mathcal{A}} \quad \text { with } \quad \phi\left(\mathcal{A}_{i}(\Sigma, M) \cap \mathcal{B}_{\Lambda, i}(\Sigma, M)\right)=\phi\left(\mathcal{A}_{i}(\Sigma, M)\right) \cap E_{\mathcal{B}},
$$

satisfying the additional condition that $\phi\left(\mathcal{A}_{i}(\Sigma, M)\right)$ be a convex subset of $E_{\mathcal{A}}$.

We emphasize that the point of introducing the submanifold $\mathcal{B}_{\Lambda}(\Sigma, M)$ is that over this submanifold, in accordance with Lemma 9.3, we have a smoothly varying family of holomorphic line bundles $\mathbf{L}$, with the holomorphic structure on each $\mathbf{L}$ being determined by the Levi-Civita connection for the metric $g \in \operatorname{Met}(M)$. Moreover, we can define $\mathcal{J}(\mathbf{L})$ and $\mathcal{J}\left(\left(f^{*} T M\right)^{\top}\right)$, spaces which vary smoothly with $(f, \omega, g) \in \mathcal{B}_{\Lambda}(\Sigma, M)$.

As in $₫ 8$, we let $\mathcal{J}_{0}(\mathbf{L})$ be the complex $n$-dimensional subspace of $\mathcal{J}(\mathbf{L})$ consisting of elements which vanish at the distinguished points $q, r$ and $s$ when $\Sigma$ is a sphere, or at the distinguished point $q$ when $\Sigma$ is a torus, while if $\Sigma$ has genus at least two, we simply set $\mathcal{J}_{0}(\mathbf{L})=\mathcal{J}(\mathbf{L})$. If $(f, \omega, g) \in \mathcal{P}_{\Lambda}$, we let $\mathcal{K}(\mathbf{L})$ be the subspace of $\mathcal{J}_{0}(\mathbf{L})$ generated by the elements $Z_{1}, Z_{2}, \ldots, Z_{n}$ constructed in Lemma 7.1, one for each branch point of $f$, and let $\mathcal{N}(\mathbf{L})$ denote the space of elements of $\mathcal{J}_{0}(\mathbf{L})$ which (like $\partial f / \partial z$ ) vanish at the branch points, so that

$$
\mathcal{J}_{0}(\mathbf{L})=\mathcal{K}(\mathbf{L}) \oplus \mathcal{N}(\mathbf{L}) .
$$

If $(f, \omega, g)$ is only an element of $\mathcal{B}_{\Lambda}(\Sigma, M)$, we define the subspaces $\mathcal{K}(\mathbf{L})$ and $\mathcal{N}(\mathbf{L})$ via principal parts, spaces of constant dimension which also vary smoothly. Finally, we let $\mathcal{J}_{0}\left(\left(f^{*} T M\right)^{\top}\right), \mathcal{K}\left(\left(f^{*} T M\right)^{\top}\right)$ and $\mathcal{N}\left(\left(f^{*} T M\right)^{\top}\right)$ denote the corresponding spaces of potential tangential Jacobi fields, so that

$$
\mathcal{J}_{0}\left(\left(f^{*} T M\right)^{\top}\right)=\mathcal{K}\left(\left(f^{*} T M\right)^{\top}\right) \oplus \mathcal{N}\left(\left(f^{*} T M\right)^{\top}\right) .
$$

It follows from Lemma 7.1 that the only elements of $\mathcal{J}_{0}\left(\left(f^{*} T M\right)^{\top}\right)$ that are perpendicular to all metric deformations are those which lie in $\mathcal{N}\left(\left(f^{*} T M\right)^{\top}\right)$. In a very rough sense, we could say that the elements of $\mathcal{K}\left(\left(f^{*} T M\right)^{\top}\right)$ can be perturbed away by variations in the metric. By construction, the spaces of potential tangential Jacobi fields $\mathcal{J}_{0}\left(\left(f^{*} T M\right)^{\top}\right), \mathcal{K}\left(\left(f^{*} T M\right)^{\top}\right)$ and $\mathcal{N}\left(\left(f^{*} T M\right)^{\top}\right)$ vary smoothly with $(f, \omega, g) \in \mathcal{B}_{\Lambda}(\Sigma, M)$.

For a given choice of $i \in I$, we can extend the family of line bundles $\mathbf{L}$ over $\mathcal{B}_{\Lambda, i}(\Sigma, M)$ to $\mathcal{A}_{i}(\Sigma, M)$ by pulling back via the projection

$$
\pi: E_{\mathcal{A}} \rightarrow E_{\mathcal{B}} \quad \text { along } \quad F
$$

in terms of the local coordinate system on $\mathcal{A}_{i}(\Sigma, M)$. In a similar way, we can pull back to $\mathcal{A}_{i}(\Sigma, M)$ the smoothly varying spaces $\mathcal{J}_{0}(\mathbf{L}), \mathcal{K}(\mathbf{L})$ and $\mathcal{N}(\mathbf{L})$ and the corresponding spaces of potential tangential Jacobi fields $\mathcal{J}_{0}\left(\left(f^{*} T M\right)^{\top}\right), \mathcal{K}\left(\left(f^{*} T M\right)^{\top}\right)$ and $\mathcal{N}\left(\left(f^{*} T M\right)^{\top}\right)$,

We now modify the procedure we used in $\oint 3$, by constructing a subbundle $\mathcal{E}$ of the restriction of $T \operatorname{Map}(\Sigma, M)$ or $T \mathcal{F}_{i}(\Sigma, M)$ to $\mathcal{A}_{i}(\Sigma, M)$. However, we now take $\mathcal{E}$ to be the bundle whose fiber over $f$ is

$\left\{X \in T_{f} \operatorname{Map}(\Sigma, M): X\right.$ is perpendicular to $\left.\operatorname{Re}(\mathcal{N}(\mathbf{L}))\right\}$

$$
\text { or }\left\{X \in T_{f} \mathcal{F}_{i}(\Sigma, M): X \text { is perpendicular to } \operatorname{Re}(\mathcal{N}(\mathbf{L}))\right\}
$$

depending on the genus of $\Sigma$, where Re denotes projection onto the real part, and let

$$
p: T \operatorname{Map}(\Sigma, M) \times T \mathcal{T} \rightarrow \mathcal{E} \times T \mathcal{T} \quad \text { or } \quad p: T \mathcal{F}_{i}(\Sigma, M) \times T \mathcal{T} \rightarrow \mathcal{E} \times T \mathcal{T}
$$


be the corresponding orthogonal bundle projections. We use the same EulerLagrange maps

$$
\begin{aligned}
F: \mathcal{A}(\Sigma, M) \longrightarrow \operatorname{Map}(\Sigma, T M) \times T \mathcal{T} \\
\text { or } \quad F: \mathcal{A}_{i}(\Sigma, M) \longrightarrow \operatorname{Map}(\Sigma, T M) \times T \mathcal{T}
\end{aligned}
$$

that we used before in $₫ 8$, with the $L_{k}^{2}$ topology on the space of maps in the domain and the $L_{k-2}^{2}$ topology on the range, for suitable choice of $k$.

Having made these preparations, we can now define our desired submanifold

$$
\mathcal{Q}_{\Lambda, i}=(p \circ F)^{-1}(\mathcal{Z})
$$

$\mathcal{Z}$ being the zero section of $\mathcal{E} \times T \mathcal{T}$. The condition that $(f, \omega, g) \in \mathcal{Q}_{\Lambda, i}$ is not that $(f, \omega, g)$ be conformal harmonic, but only that

$$
\frac{1}{\lambda^{2}}\left[\frac{D^{g}}{\partial x_{1}}\left(\frac{\partial f}{\partial x_{1}}\right)+\frac{D^{g}}{\partial x_{2}}\left(\frac{\partial f}{\partial x_{2}}\right)\right] \in \operatorname{Re}(\mathcal{N}(L))
$$

and

$$
P\left(\left\langle\frac{\partial f}{\partial z}, \frac{\partial f}{\partial z}\right\rangle d z^{2}\right)=0
$$

where $P$ is once again the orthogonal projection to the space of holomorphic quadratic differentials. Thus we could say that $(f, \omega, g)$ is harmonic up to a finitedimensional error.

To show that $p \circ F$ is transversal to the zero section of $\mathcal{E} \times \mathcal{T}$, we must show that at a point $(f, \omega, g) \in \mathcal{Q}_{\Lambda, i}$, the space $\mathcal{V}=0$, where

$$
\begin{aligned}
\mathcal{V}=\left\{(X, \dot{\omega}): X \text { is a section of } \mathcal{E}, \dot{\omega} \in T_{\omega} \mathcal{T}\right. \\
\\
\text { and } \left.(X, \dot{\omega}) \text { is perpendicular to the image of } \pi_{V} \circ D(p \circ F)_{(f, \omega, g)}\right\} .
\end{aligned}
$$

Any element of $\mathcal{V}$ must lie in the kernel of the restricted Jacobi operator

$$
\begin{aligned}
& L^{\prime}=\pi_{V} \circ D_{1}(p \circ F)_{(f, \omega, g)} \mid \mathcal{E}_{(f, \omega)}: \\
& \quad\left(L_{k}^{2} \text {-sections of } \mathcal{E}_{(f, \omega)}\right) \rightarrow\left(L_{k-2}^{2} \text {-sections of } \mathcal{E}_{(f, \omega)}\right) .
\end{aligned}
$$

Note that this operator is defined even if $(f, \omega, g)$ is only a zero for $p \circ F$, not for $F$ itself.

By passing to a refinement of the covering if necessary, we can assume that $\mathcal{W}_{i}$ is contained in an $\epsilon$-neighborhood in the $L_{k}^{2}$ topology about some point $\left(f_{0}, \omega_{0}, g_{0}\right) \in$ $\mathcal{P}_{\Lambda}(\Sigma, M)$, where $\epsilon>0$ depends upon the spectrum of the elliptic operator $L^{\prime}$. (We can assume, moreover, that $\left(f_{0}, \omega_{0}, g_{0}\right) \notin \mathcal{P}_{\Lambda^{\prime}}(\Sigma, M)$ for $\Lambda^{\prime}<\Lambda$, and choose the neighborhood so small that points within it have an intersection set that is no worse than that of $\left(f_{0}, \omega_{0}, g_{0}\right)$.) At $\left(f_{0}, \omega_{0}, g_{0}\right)$, the operator $L^{\prime}$ has a finitedimensional kernel $K$ which contains the space $\mathcal{K}\left(\left(f^{*} T M\right)^{\top}\right)$ of tangential Jacobi fields corresponding to $\mathcal{K}(\mathbf{L})$. Suppose that

$$
\left\{\left(X_{1}, \dot{\omega}_{1}\right), \ldots,\left(X_{m}, \dot{\omega}_{m}\right)\right\} \quad \text { and } \quad\left\{\left(X_{1}, \dot{\omega}_{1}\right), \ldots,\left(X_{n}, \dot{\omega}_{n}\right)\right\}
$$

are real bases for $\mathcal{K}\left(\left(f^{*} T M\right)^{\top}\right)$ and $K$ respectively, with $m \leq n$. By the argument for Lemma 8.1, which in turn is based on Lemma 7.1, we can find perturbations in the metric $h_{1}, \ldots, h_{m}$ in $T_{g_{0}} \operatorname{Met}(M)$ such that

$$
\operatorname{Re}\left\langle\pi_{V} \circ\left(D_{2} F\right)_{\left(f_{0}, \omega_{0}, g_{0}\right)}\left(h_{a}\right),\left(X_{b}, \dot{\omega}_{b}\right)\right\rangle= \begin{cases}1, & \text { if } a=b, \\ 0, & \text { if } a \neq b,\end{cases}
$$


with the range of indices $1 \leq a, b \leq m, X$ being a real section of $f^{*} T M$. Recall that the metric variations $h_{1}, \ldots, h_{m}$ correspond to meromorphic quadratic differentials on $\Sigma$ which have simple poles at the branch points (and are suitably regularized). On the other hand, since the elements $\left(X_{r}, \dot{\omega}_{r}\right)$, for $m+1 \leq r \leq n$, are not Jacobi fields (because $\operatorname{Re}\left(\left(f^{*} T M\right)^{\top}\right)$ goes to zero under the projection to $\left.\mathcal{E}\right)$, it follows from the argument for Lemma 6.1 that we can construct additional perturbations in the metric $h_{m+1}, \ldots, h_{n}$ in $T_{g_{0}} \operatorname{Met}(M)$, such that

$$
\operatorname{Re}\left\langle\pi_{V} \circ\left(D_{2} F\right)_{\left(f_{0}, \omega_{0}, g_{0}\right)}\left(h_{i}\right),\left(X_{j}, \dot{\omega}_{j}\right)\right\rangle= \begin{cases}1, & \text { if } i=j, \\ 0, & \text { if } i \neq j,\end{cases}
$$

the range of indices now being $1 \leq i, j \leq n$. From this it follows that $\mathcal{V}=0$ at $\left(f_{0}, \omega_{0}, g_{0}\right)$. Note that the matrix of these inner products continues to be nonsingular for $(f, \omega, g)$ sufficiently close to $\left(f_{0}, \omega_{0}, g_{0}\right)$ when $\left(X_{1}, \dot{\omega}_{1}\right), \ldots,\left(X_{n}, \dot{\omega}_{n}\right)$ and $h_{1}, \ldots, h_{n}$ are perturbed by a small amount.

For $(f, \omega, g)$ sufficiently close to $\left(f_{0}, \omega_{0}, g_{0}\right)$, the kernel of the operator $L^{\prime}$ defined by (94) will be a subspace of a linear space $K^{\prime}(f, \omega, g)$ which is close to $K$ and of the same dimension, a linear space which varies smoothly with $(f, \omega, g)$. (The space $K^{\prime}(f, \omega, g)$ is generated by eigenvalues near zero of the operator $L^{\prime}$.) We can replace $\left\{\left(X_{1}, \dot{\omega}_{1}\right), \ldots,\left(X_{n}, \dot{\omega}_{n}\right)\right\}$ by a basis for $K^{\prime}(f, \omega, g)$ and $h_{1}, \ldots, h_{n}$ by small perturbations without destroying the nonsingularity of the matrix on the left-hand side of (95). It follows that no nontrivial element of the kernel of $L^{\prime}$ can lie in $\mathcal{V}$, and hence $\mathcal{V}$ must be zero at the point $(f, \omega, g) \in \mathcal{W}_{i}$, whenever $p \circ F(f, \omega, g)=0$, if $\mathcal{W}_{i}$ has been chosen to be an $\epsilon$-neighborhood of $\left(f_{0}, \omega_{0}, g_{0}\right)$, for $\epsilon>0$ sufficiently small.

Now just as in the arguments for Lemmas 3.1, 6.1 and 8.1, we see that the restriction of the map $F$ defined by (93) to $\mathcal{W}_{i}$ is transversal to the zero section, and hence $\mathcal{Q}_{\Lambda, i}$ is indeed a submanifold of $\mathcal{W}_{i}$.

Moreover, we claim that the arguments for Lemmas 3.2, 6.2 and 8.2 show that

$$
\pi: \mathcal{Q}_{\Lambda, i} \longrightarrow \operatorname{Met}(M)
$$

is Fredholm and $d \pi$ is not surjective at points of $\mathcal{Q}_{\Lambda, i}$. Indeed, in analogy with (84), we find that the tangent space to $\mathcal{Q}_{\Lambda, i}$ is

$$
\begin{aligned}
T_{(f, \omega, g)} \mathcal{Q}_{\Lambda, i}=\left\{(X, \dot{\omega}, h) \in T_{f} \operatorname{Map}(\Sigma, M) \oplus T_{\omega} \mathcal{T} \oplus T_{g} \operatorname{Met}(M):\right. \\
\left.L^{\prime}(X, \dot{\omega})+p \circ \pi_{V} \circ\left(D_{2} F\right)_{(f, \omega, g)}(h)=0\right\},
\end{aligned}
$$

$p$ being the projection into the bundle $\mathcal{E}$ which has codimension equal to the dimension of $\mathcal{N}\left(\left(f^{*} T M\right)^{\top}\right)$. If $(X, \dot{\omega})$ is a tangential Jacobi field, we can define a linear functional on $T_{g} \operatorname{Met}(M)$ by

$$
\begin{aligned}
& T(X, \dot{\omega}): T_{g} \operatorname{Met}(M) \rightarrow \mathbb{R} \\
& \text { by } \quad T(X, \dot{\omega})(h)=\operatorname{Re}\left\langle\left\langle\pi_{V} \circ\left(D_{2} F\right)_{(f, \omega g)}(h),(X, \dot{\omega})\right\rangle\right\rangle .
\end{aligned}
$$

Lemma 7.1 shows that when $(X, \dot{\omega}) \in \mathcal{K}\left(\left(f^{*} T M\right)^{\top}\right)$, this linear functional is nonzero. But by definition of the tangent space to $\mathcal{Q}_{\Lambda, i}, T(X, \dot{\omega})$ must vanish on the range of $d \pi$, when $(X, \dot{\omega})$ is Jacobi. Indeed, it follows from Lemma 7.1 that there are at least two such linear functionals, linearly independent over the reals, and hence the cokernel of $d \pi$ has dimension at least two. The verification that $d \pi$ is Fredholm is straightforward.

This finishes the proof of Lemma 10.1 and the proof of the Main Theorem. 


\section{NonORIENTABLE SURFACES}

Any complete theory of minimal surfaces must take nonorientable surfaces into account. Indeed, the first applications we have in mind are to minimal spheres and tori, and these can cover prime minimal projective planes and Klein bottles. In particular, we will need to be able to recognize when a minimal torus covers a minimal Klein bottle. Therefore we give a brief description of how the preceding results can be modified to treat nonorientable surfaces, such as projective planes and Klein bottles.

First, we note that the lemmas of $\$ 4$ apply directly to nonorientable surfaces. However, in the rest of the argument, much of which utilizes complex analysis, it is expedient to pass from the nonorientable surfaces themselves to their orientable double covers. We describe briefly how this works, with emphasis on projective planes and Klein bottles.

A minimal projective plane can be regarded as a conformal harmonic map

$$
f: S^{2} \longrightarrow M \text { such that } f \circ A=f,
$$

where $A: S^{2} \rightarrow S^{2}$ is the antipodal map. (From the point of view of conformal geometry, there are many antipodal maps, but once we have reduced the symmetry group to $S O(3)$ by requiring that the center of mass is zero, as described in (2) in the Introduction, there is a canonical one that is an isometry with respect to our canonical choice of Riemannian metric on $S^{2}$.) To put this in variational form, we note that the energy is invariant under composition of $f$ with $A$, so to find minimal projective planes, we need only find critical points for the restriction of the energy to the space

$$
\operatorname{Map}_{0}\left(\mathbb{R} P^{2}, M\right)=\left\{f \in \operatorname{Map}_{0}\left(S^{2}, M\right): f \circ A=f\right\}
$$

and restrict the energy to a function

$$
E: \operatorname{Map}_{0}\left(\mathbb{R} P^{2}, M\right) \longrightarrow \mathbb{R} .
$$

The action of $A$ on $\operatorname{Map}_{0}\left(S^{2}, M\right)$, defined by $A(f)=f \circ A$, commutes with the action of $S O(3)$ in this case, so the energy (96) is $S O(3)$-invariant.

Similarly, a minimal Klein bottle will be covered by a minimal torus with a flat metric of area one that is invariant under an orientation-reversing deck transformation $A_{s}$, for $s \in S^{1}$, which consists of a translation composed with a reflection, expressed in terms of appropriate standard coordinates $\left(t_{1}, t_{2}\right)$ on the torus as

$$
A_{s}\left(t_{1}, t_{2}\right)=\left(t_{1}+1 / 2,-t_{2}-s\right),
$$

which one easily checks satisfies the identity $A_{s}^{2}=1$. Recall that the Teichmüller space $\mathcal{T}_{1}$ for the torus is the upper half-plane

$$
\mathbb{H}=\{\omega=u+i v \in \mathbb{C}: v>0\},
$$

with the point $\omega=u+i v$ corresponding to the conformal class of the torus $\mathbb{C} / \Lambda$, and where $\Lambda$ is the lattice in $\mathbb{C}$ generated by 1 and $\omega$. In the case of a double cover of a Klein bottle, we arrange that the differential of $A_{s}$ fixes the generator corresponding to 1 in the fundamental parallelogram, and the orthogonal line in the complex plane must then be preserved under the reflection $u+i v \mapsto u-i v$, so the "Teichmüller space" of flat Klein bottles with total area one consists of the positive real numbers $\omega=i v$ with $v>0$, the fixed point set of the involution

$$
A_{\star}: \mathbb{H} \longrightarrow \mathbb{H}, \quad A_{\star}(u+i v)=-u+i v .
$$


(See Wolf 39], Proposition 2.5.8, for example.) If we let

$$
\operatorname{Map}\left(K^{2}, M\right)=\left\{f \in \operatorname{Map}\left(T^{2}, M\right): f \circ A_{s}=f \text { for some } s \in S^{1}\right\},
$$

minimal Klein bottles can then be regarded as critical points for the restricted two-variable function

$$
E: \operatorname{Map}\left(K^{2}, M\right) \times\left\{\omega \in \mathcal{T}_{1}: A_{\star}(\omega)=\omega\right\} \longrightarrow \mathbb{R} .
$$

The energy is invariant under the action of $S^{1} \times S^{1}$ on $\operatorname{Map}\left(K^{2}, M\right)$ defined by

$$
f\left(t_{1}, t_{2}\right) \mapsto f\left(t_{1}+s_{1}, t_{2}+s_{2}\right), \quad \text { for } \quad\left(s_{1}, s_{2}\right) \in S^{1} \times S^{1} .
$$

In the subsequent discussion, we let $A=A_{s_{0}}$ for some choice of $s_{0} \in S^{1}$, thereby breaking part of the $S^{1} \times S^{1}$-symmetry.

If $f_{0}: \Sigma_{0} \rightarrow M$ is a nonorientable parametrized minimal surface with oriented double cover $f: \Sigma \rightarrow M$ of genus at least two, we let $A: \Sigma \rightarrow \Sigma$ be the sheet interchange map.

Suppose now that $f: \Sigma \rightarrow M$ is an oriented double cover of a nonorientable minimal surface $f_{0}: \Sigma_{0} \rightarrow M$. The map $A$ induces an involution on $A_{\star}$ on $f^{*} T M$, as well as on the space of sections of $f^{*} T M$, and both of these actions extend complex linearly to the complexifications. Moreover, these involutions preserve both the metric and the pullback of the Levi-Civita connection. If $\mathbf{E}=f^{*} T M \otimes \mathbb{C}$, we can therefore construct a direct sum decomposition $\Gamma(\mathbf{E})=\Gamma_{+}(\mathbf{E}) \oplus \Gamma_{-}(\mathbf{E})$, where

$$
\Gamma_{+}(\mathbf{E})=\left\{X \in \Gamma(\mathbf{E}): A_{\star}(X)=\bar{X}\right\}, \quad \Gamma_{-}(\mathbf{E})=\left\{X \in \Gamma(\mathbf{E}): A_{\star}(X)=-\bar{X}\right\} .
$$

The sections of $\Gamma_{+}(\mathbf{E})$ can be regarded as deformations of the nonorientable minimal surface $f_{0}: \Sigma \rightarrow M$. The argument presented in 33 shows that if $f_{0}: \Sigma \rightarrow M$ is a somewhere injective conformal harmonic map from a nonorientable surface to $M$, nontangential elements of $\Gamma_{+}(\mathbf{E})$ can be perturbed away by variations of the metric on $M$. Moreover, the proof of Proposition 3.1 can be modified to apply to somewhere injective nonorientable harmonic maps, so long as the line bundle $\mathbf{L}$ on the orientable double cover has only the holomorphic sections demanded by the group of symmetries.

To carry out the second variation formula in $\$ 5$, we need to define a Teichmüller space $\mathcal{T}_{\Sigma_{0}}$ for the nonorientable surface $\Sigma_{0}$. Of course, for the projective plane the Teichmüller space is trivial, while for the Klein bottle, we have seen that the Teichmüller space is

$$
\mathcal{T}_{K^{2}}=\left\{\omega \in \mathcal{T}_{1}: A_{\star}(\omega)=\omega\right\} .
$$

For surfaces of higher genus, we can define the Teichmüller space of $\Sigma_{0}$ as the quotient

$$
\mathcal{T}_{\Sigma_{0}}=\frac{\operatorname{Met}_{0}\left(\Sigma_{0}\right)}{\operatorname{Diff}_{0}\left(\Sigma_{0}\right)},
$$

where $\operatorname{Met}_{0}\left(\Sigma_{0}\right)$ is the space of constant curvature metrics of total area one and $\operatorname{Diff}_{0}\left(\Sigma_{0}\right)$ is the space of diffeomorphisms of $\Sigma_{0}$ which are isotopic to the identity, just as in the orientable case. It is easily checked that this agrees with the definition we have given in the case of the Klein bottle.

When $\Sigma_{0}$ is a nonorientable surface whose orientable double cover $\Sigma$ has genus $g \geq 2$, we can also describe the Teichmüller space $\mathcal{T}_{\Sigma_{0}}$ of $\Sigma_{0}$ as a subspace of the Teichmüller space $\mathcal{T}_{\Sigma}$ of $\Sigma$. To see this, note first that $A_{\star}$ induces a conjugate linear map on quadratic differentials on $\Sigma$, and the cotangent space to $\mathcal{T}_{\Sigma_{0}}$ should be the 
space of holomorphic quadratic differentials $h d z^{2}$ on $\Sigma$ such that $A_{\star}\left(h d z^{2}\right)=\bar{h} d \bar{z}^{2}$. As described in Chapter 2, $\S 3$ of [34], once one picks an origin $\eta_{0} \in \mathcal{T}_{\Sigma}$, there is a bijection $\mathcal{H}$ from $\mathcal{T}_{\Sigma}$ to the space of holomorphic quadratic differentials on $\left(\Sigma, \omega_{0}\right)$, where $\omega_{0}$ is the conformal equivalence class of $\eta_{0}$ defined as follows: If $\eta$ is a hyperbolic metric of area one on $\Sigma$, then $\mathcal{H}(\eta)$ is the Hopf differential of the harmonic map from $\left(\Sigma, \eta_{0}\right)$ to $(\Sigma, \eta)$, which exists by a theorem of Eells and Sampson and is unique by a theorem of Hartman. This isomorphism allows us to transfer $A_{\star}$ to an involution on the Teichmüller space $\mathcal{T}_{\Sigma}$, the fixed point set of the involution being exactly those hyperbolic metrics which are preserved by the deck transformation $A$ and thus descend to the nonorientable surface $\Sigma_{0}$. Hence we can set

$$
\mathcal{T}_{\Sigma_{0}}=\left\{\omega \in \mathcal{T}_{\Sigma}: A_{\star}(\omega)=\omega\right\} .
$$

Once we have properly defined the Teichmüller space for the nonorientable surface $\Sigma$, we can calculate the second variation of the energy

$$
E: \operatorname{Map}\left(\Sigma_{0}, M\right) \times \mathcal{T}_{\Sigma_{0}} \longrightarrow \mathbb{R},
$$

by applying the formula of $\$ 5$ to the oriented double cover. In the second variation for $\Sigma_{0}$, the holomorphic differentials, or the corresponding Beltrami differentials, must be invariant under the conjugate linear involution $A_{\star}$. Since $A$ is orientation reversing,

$$
\frac{\partial f}{\partial z} d z \mapsto \frac{\partial f}{\partial \bar{z}} d \bar{z}
$$

under $A_{\star}$, so $A_{\star}$ exchanges the line bundle $\mathbf{L}$ over the orientable double cover of $\Sigma_{0}$ with its conjugate. The tangential Jacobi fields are now isomorphic to the pairs $\left(Z, h d z^{2}\right)$, where $Z$ is a section of $\mathbf{L}$ and $h d z^{2}$ is a holomorphic quadratic differential, which satisfy (59) as well as the identities

$$
A_{\star}(Z)=\bar{Z}, \quad A_{\star}\left(h d z^{2}\right)=\bar{h} d \bar{z}^{2} .
$$

We define

$$
\mathcal{O}_{A}(\mathbf{L})=\left\{X \in \mathcal{O}(\mathbf{L}): A_{\star}(X)=\bar{X}\right\}, \quad \mathcal{J}_{A}(\mathbf{L})=\left\{X \in \mathcal{J}(\mathbf{L}): A_{\star}(X)=\bar{X}\right\}
$$

as real linear subspaces of $\mathcal{O}(\mathbf{L})$ and $\mathcal{J}(\mathbf{L})$, respectively. The elements of $\mathcal{J}_{A}(\mathbf{L})$ correspond to tangential Jacobi fields which generate deformations of the nonorientable minimal surface. It is now straightforward to verify that Proposition 6.1 holds for nonorientable surfaces without branch points.

Each branch point of the nonorientable minimal surface $f_{0}: \Sigma_{0} \rightarrow M$ gives rise to two branch points of the orientable double cover $f$ which are interchanged by $A$, so if $\nu$ is the total branching order of $f_{0}$, the total branching order of $f$ will be $2 \nu$. One can check that in the case of real projective planes, the real dimension of $\mathcal{O}_{A}(\mathbf{L})$ will be $3+2 \nu$, while in the case of Klein bottles, the real dimension of $\mathcal{J}_{A}(\mathbf{L})$ will be $1+2 \nu$.

The argument for the Main Theorem can now be extended so that it applies to nonorientable surfaces. In the proof, we need a Teichmüller space for the punctured nonorientable surface

$$
\Sigma_{0}-\left\{\text { branch points } p_{1}, \ldots, p_{n}\right\} .
$$

Each branch point $p_{i}$ lifts to two branch points $\tilde{p}_{i}$ and $\hat{p}_{i}$ in the oriented double cover which are interchanged by $A$. If $\Sigma$ has genus at least two, the cotangent space to the Teichmüller space for $\Sigma_{0}$ consists of the meromorphic quadratic differentials 
$h d z^{2}$ on the oriented double cover with at most simple poles at the branch points $\tilde{p}_{i}$ and $\hat{p}_{i}$ such that

$$
A_{\star}\left(h d z^{2}\right)=\bar{h} d \bar{z}^{2} .
$$

In the case where $\Sigma=T^{2}$, we excise one additional pair of points from $T^{2}$ which are interchanged by $A$, while if $\Sigma=S^{2}$, we excise three pairs of points interchanged by $A$. The cotangent space to Teichmüller space for the new punctured surface consists of the real linear subspace of the space of meromorphic quadratic differentials on the oriented double cover which are invariant under $A$ and have at most simple poles at the branch points and excised points.

Once these changes are made, it is relatively easy to check that the previous arguments yield a version of the Main Theorem for nonorientable minimal surfaces. For generic choice of metric, prime minimal projective planes and Klein bottles are free of branch points and lie on nondegenerate critical submanifolds with dimensions dictated by the group actions $\left(S O(3)\right.$ or $\left.S^{1} \times S^{1}\right)$. For generic choice of metric, prime nonorientable minimal surfaces whose orientable double covers have genus at least two are Morse nondegenerate in the usual sense.

We can now give a rough description of the full collection of parametrized minimal spheres and tori in a Riemannian manifold with generic metric. For minimal two-spheres, we first have the constant maps which make up a nondegenerate critical submanifold at energy level zero. Then we have the prime minimal two-spheres themselves. Next we have the branched covers of minimal two-spheres, which can be thought of as more complicated parametrizations of the prime minimal twospheres. Finally, we have prime minimal projective planes as well as covers of these, including two-to-one covers of projective planes in which the parameter space is the two-sphere and branched covers of prime minimal projective planes.

For the tori, we have the constant maps and the prime minimal tori once again. This time, however, there are two types of covers, ordinary covers of prime minimal tori and Klein bottles without branch points, and branched covers of prime minimal two-spheres and projective planes.

When the genus is at least two, a complete critical point theory for minimal surfaces of genus $g$ would have to consider prime surfaces of genus $g$, branched covers of prime minimal surfaces of genus $\leq g$ and branched covers of prime nonorientable surfaces.

\section{FAMILIES OF METRICS}

Finally, we would like to describe an extension of the Main Theorem (which can be extended to nonorientable surfaces if desired) to one-parameter families of Riemannian metrics on $M$.

Recall that for each branch type $\Lambda$ we have a subspace $\mathcal{P}_{\Lambda}$ of $\mathcal{A}(\Sigma, M)$ consisting of prime parametrized minimal surfaces with energy less than a fixed bound $E_{0}$ and branch type $\Lambda$. (See (92) from \$10) In the case where $\Lambda=\emptyset$, the elements of $\mathcal{P}_{\Lambda}=\mathcal{P}_{\emptyset}$ are immersions and $\mathcal{P}_{\emptyset}$ is a submanifold of $\mathcal{A}(\Sigma, M)$ with Fredholm projection

$$
\pi: \mathcal{P}_{\emptyset} \longrightarrow \operatorname{Met}(M)
$$

of Fredholm index $d_{\Sigma}$, where $d_{\Sigma}$ is the real dimension of the group of symmetries. Moreover, $\pi^{-1}\left(g_{0}\right)$ is a submanifold of dimension $d_{\Sigma}$, each component being diffeomorphic to the group $G$ of symmetries, for generic choice of $g_{0} \in \operatorname{Met}(M)$. 
On the other hand, if $\Lambda$ has positive degree, then $\mathcal{P}_{\Lambda}$ is generated by the $G$ orbits of the union of subsets of a countable collection of submanifolds $\mathcal{Q}_{\Lambda, i}$ of $\mathcal{A}(\Sigma, M)$ and $d \pi$ has cokernel of dimension at least two at each point of each $\mathcal{Q}_{\Lambda, i}$ by Lemma 10.1 .

Suppose now that $g_{0}$ and $g_{1}$ are generic metrics and $\gamma:[0,1] \rightarrow \operatorname{Met}(M)$ is a smooth path with $\gamma(0)=g_{0}$ and $\gamma(1)=g_{1}$. By Theorem 3.1 of [35, we can construct a smooth approximation $\tilde{\gamma}$ to $\gamma$ with $\tilde{\gamma}(0)=g_{0}$ and $\tilde{\gamma}(1)=g_{1}$ such that if $\pi$ is the projection to $\operatorname{Met}(\Sigma)$, then $\tilde{\gamma}$ is transversal to $\pi$, for each $\mathcal{P}_{\Lambda}$ or each $\mathcal{Q}_{\Lambda, i}$, depending on the branching type. This leads to the following conclusion.

Theorem 12.1. Let $M$ be a compact connected smooth manifold of dimension at least three. A generic path $\gamma:[0,1] \rightarrow \operatorname{Met}(M)$ between generic metrics on $M$ will have the property that $\pi^{-1}(\gamma([0,1]))$ contains only minimal surfaces $f: \Sigma \rightarrow M$ with no branch points. Moreover $\pi^{-1}(\gamma([0,1]))$ will be a smooth submanifold of dimension $d_{\Sigma}+1$ of

$$
\begin{aligned}
\mathcal{P}_{\emptyset}=\{(f, \omega, g) \in \operatorname{Map}(\Sigma, M) \times \mathcal{T} \times \operatorname{Met}(M): \\
\quad f \text { is a prime minimal immersion, conformal with respect to } \omega \text { and } g\} .
\end{aligned}
$$

Here $d_{\Sigma}$ is the dimension of the group $G$ of symmetries of the energy E.

\section{REFERENCES}

[1] W. Abikoff, The real analytic theory of Teichmüller space, Lecture Notes in Mathematics no. 820, Springer-Verlag, New York, 1980. MR0590044 (82a:32028)

[2] R. Abraham, Lectures of Smale on differential topology, Lecture notes from Columbia University, 1963.

[3] R. Abraham, Bumpy metrics, Proc. Symp. Pure Math. 14 (1970), 1-3. MR0271994(42:6875)

[4] R. Abraham, J. Marsden and T. Ratiu, Manifolds, tensor analysis, and applications, Second Edition, Addison-Wesley, 1988.

[5] R. Abraham and J. Robbin, Transversal mappings and flows, Benjamin, New York, 1967. MR0240836 (39:2181)

[6] N. Aronszajn, A unique continuation theorem for solutions of elliptic partial differential equations or inequalities of second order, J. Math. Pure Appl. 36 (1957), 235-249. MR0092067 $(19: 1056 \mathrm{c})$

[7] L. Bers, Finite dimensional Teichmüller spaces and generalizations, Bull. Amer. Math. Soc. 5 (1981), 131-172. MR0621883 (82k:32050)

[8] R. Böhme and A. Tromba, The index theorem for classical minimal surfaces, Annals of Math. 113 (1981), 447-499. MR0621012 (83a:58031)

[9] R. Böhme and A. Tromba, The index theorem for minimal surfaces of higher genus, Memoirs of the Amer. Math. Soc. 560 (1995).

[10] R. Bott, Lectures on Morse theory, old and new, Bull. Amer. Math. Soc. 7 (1982), 331-358. MR0663786 (84m:58026a)

[11] S. Y. Cheng, Eigenfunctions and nodal sets, Comm. Math. Helv. 51 (1976), 43-55. MR0397805 (53:1661)

[12] C. Earle and J. Eells, A fibre bundle description of Teichmüller theory, J. Differential Geometry 3 (1969), 19-43. MR.0276999(43:2737a)

[13] J. Eells, A setting for global analysis, Bull. Amer. Math. Soc. 72 (1966), 751-807. MR0203742 $(34: 3590)$

[14] A. E. Fischer and A. J. Tromba, On a purely "Riemannian" proof of the structure and dimension of the unramified moduli space of a compact Riemann surface, Math. Ann. 267 (1984), 311-345. MR0738256 (85m:58045)

[15] D. Freed and K. Uhlenbeck, Instantons and four-manifolds, 2nd edition, Springer, New York, 1991. MR.1081321 (91i:57019)

[16] M. Freedman, J. Hass and P. Scott, Least area incompressible surfaces in three-manifolds, Inventiones Math. 71 (1983), 609-642. MR0695910(85e:57012) 
[17] R. Gulliver, R. Osserman and H. Royden, A theory of branched immersions, Amer. J. Math. 95 (1973), 750-812. MR0362153(50:14595)

[18] R. Gunning, Lectures on Riemann surfaces, Princeton Univ. Press, Princeton NJ, 1966. MR0207977 (34:7789)

[19] N. Hingston, Equivariant Morse theory and closed geodesics, J. Differential Geometry 19 (1978), 85-116. MR0739783 (85i:58036)

[20] W. Klingenberg, Lectures on closed geodesics, Springer, New York, 1978. MR0478069 $(57: 17563)$

[21] S. Lang, Differential and Riemannian manifolds, Springer, New York, 1995. MR.1335233 (96d:53001)

[22] O. Lehto, Univalent functions and Teichmüller space, Springer, New York, 1987. MR0867407 (88f:30073)

[23] D. McDuff and D. Salamon, J-holomorphic curves and symplectic topology, Amer. Math. Soc., Providence, Rhode Island, 2004. MR2045629 (2004m:53154)

[24] W. Meeks and S. T. Yau, Topology of three-dimensional manifolds and the embedding problems in minimal surface theory, Annals of Math. 112 (1980), 441-484. MR0595203 (83d:53045)

[25] W. Meeks and S. T. Yau, The classical Plateau problem and the topology of three-dimensional manifolds: the embedding of the solution given by Douglas-Morrey and an analytic proof of Dehn's Lemma, Topology 21 (1982), 409-442. MR0670745 (84g:53016)

[26] M. Micallef and J. D. Moore, Minimal two-spheres and the topology of manifolds with positive curvature on totally isotropic two-planes, Annals of Math. 127 (1988), 199-227. MR0924677 (89e:53088)

[27] M. Micallef and B. White, The structure of branch points in minimal surfaces and pseudoholomorphic curves, Annals of Math. 141 (1995), 35-85. MR.1314031 (96a:58063)

[28] J. D. Moore, On the number of minimal two-spheres of small area in manifolds with curvature bounded above, Math. Ann. 288 (1990), 323-343. MR1075771(91i:58029)

[29] R. Osserman, A survey of minimal surfaces, Dover, Mineola, New York, 1986. MR 0852409 (87j:53012)

[30] R. Palais, Foundations of global nonlinear analysis, Benjamin, New York, 1968. MR0248880 $(40: 2130)$

[31] J. Sacks and K. Uhlenbeck, The existence of minimal immersions of 2-spheres, Annals of Math. 113 (1981), 1-24. MR0604040 (82f:58035)

[32] J. Sacks and K. Uhlenbeck, Minimal immersions of closed Riemann surfaces, Trans. Amer. Math. Soc. 271 (1982), 639-652. MR0654854 (83i:58030)

[33] J. H. Sampson, Some properties and applications of harmonic mappings, Annales Scientifiques de l'École Normale Supérieure 11 (1978), 211-228. MR0510549(80b:58031)

[34] R. Schoen and S. T. Yau, Lectures on harmonic maps, International Press, Boston, 1997. MR.1474501 (98i:58072)

[35] S. Smale, An infinite-dimensional version of Sard's theorem, Amer. J. Math. 87 (1966), 861-866. MR0185604 (32:3067)

[36] K. Uhlenbeck, Integrals with nondegenerate critical points, Bull. Amer. Math. Soc. 76 (1970), 125-128. MR.0254873(40:8080)

[37] K. Uhlenbeck, Morse theory on Banach manifolds, J. Functional Analysis 10 (1972), 430-445. MR 0377979 (51:14148)

[38] B. White, The space of minimal submanifolds for varying Riemannian metrics, Indiana Math. J. 40 (1991), 161-200. MR1101226 (92i:58028)

[39] J. Wolf, Spaces of constant curvature, McGraw-Hill, New York, 1967. MR0217740 (36:829)

Department of Mathematics, University of California, Santa Barbara, California 93106

E-mail address: moore@math.ucsb.edu 